\title{
Flowers and Insects in Great Britain.
}

\author{
PART IV. 1
}

\section{Observations on the less Specialized Flowers of the Clova Mountains.}

BY

\author{
J. C. WILLIS, Sc.D., \\ Director of the Royal Botanic Gardens, Ceylon,
}

AND

I. H. BURKILL, M.A.,

Officiating Reporter on Economic Products to the Government of India.

HIS part of our paper contains all our unpublished observations upon
Clova flowers; it will be followed shortly by a concluding part, wherein our results will be reviewed.

\section{Class B, $\S 29$. Large Crucifer Type.}

I48. Cheiranthus Cheiri, Linn. [Lit. Brit. 23,29 ; N.C.E. 3 a, 14 a, 33, 34; Alps 34.] A garden-plant with homogamous flowers needing insect aid for fertilization, visited in Britain and Germany by Apis and Bombus. Knuth, quoting Schletterer, names a number of other Hymenoptera seen on its flowers in the Tyrol.

Visitors. Hymenoptera. Aculeata: Apidae: (I) Apis mellifica L., sh. 23. V. $97,800 \mathrm{ft}$.

149. Cardamine pratensis, Linn. [Lit. Brit. 23 ; N.C.E. 1, 3 a, 14, 18, 24, 25, 34, 40, Hildebrand 1065, de Vries 2460, Warnstorf 2507; Arct. 7, 37 a, 38.] Müller, MacLeod, Knuth, de Vries, and Alf ken record bees as visiting this plant in North Central Europe ; our observations are in singular contrast ; for out of II 5 insects seen in spring and summer on the flowers,

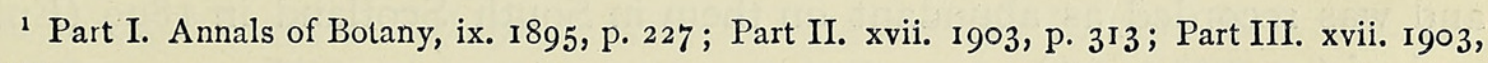
p. 539 .

Annals of Botany, Vol. XXIr. No. LXXXVIII. October, 1908.] 
102 were short-tongued flies; and there was not a single bee. The range of the plant extends far north, and its reproduction in Greenland is chiefly asexual by bulbils. The flower is variable in the relative lengths of stamens and pistil, and the probability of spontaneous self-pollination is considerable, Warming thinks very likely in Greenland, but Hildebrand considers the plant in Germany self-sterile.

Visitors. Lepidoptera. Rhopalocera: (I) Pieris napi L., II-I5. VI. 99, $800 \mathrm{ft}$. Heterocera : Eriocephalidae: (2) Eriocephala calthella L., I-2. VII. 95, 8-I,100 ft. Diptera. Syrphidae: (3) Melanostoma dubium Ztt., fp. 22. V. 96, 19-2,000 ft. Empidae: (4) Empis bilineata Lw., sh. 2 r. V. 96, $800 \mathrm{ft}$. Anthomyiidae: (5) Trichophthicus sp., fp. 29. VI. 96, r,300 ft. (6) Anthomyia sulciventris Ztt., fp. 22. V. 96, 19-2,100 ft.; 22-25. V. 97, 7-800 ft. (7 and 8) A. spp., sh. by base in withering flowers and fp. 14-22. V. $96 ; 19$. V. $97 ;$ 14. V. 98 ; 10-I 5. VI. 99, 8-2,100 ft. Helomyzidae: (9) Tephrochlamys sp., fp. 9. V. 98, $800 \mathrm{ft}$. Coleoptera. (Io) Meligethes viridescens F., 2 I. V. 96, $800 \mathrm{ft}$. (I I) Anthobium minutum F., fp. 2. VII. $95,800 \mathrm{ft}$.

I50. Arabis caucasica, Willd. [Lit. Brit. 29.] In cultivation. Apis visits it much.

Visitors. Lepidoptera. Rhopalocera: (I) Vanessa urticae L., sh. I 5-I 7. IV. 95; 23. V. 97. Hymenoptera. Aculeata: Apidae: (2) Apis mellifica L., sh. 2-1 7 . IV. $95 ; 7^{-1} 5$. V. 98 . freq. (3) Bombus terrestris L., sh. I $5^{-17}$. IV. 95 . (4) B. lapponicus F., sh. 20. V. 97. Diptera. Muscidae: (5) Lucilia cornicina F., sh. I6. IV. 95. (6) Pollenia rudis F., sh. 2. IV. 95. Anthomyiidae: (7) Anthomyia sulciventris Ztt., 23-24. V. 97. (8) A. sp., fp. I 5. V. 98. Cordyluridae: (9) Scatophaga sp., sh. 16. IV. 95. All at $800 \mathrm{ft}$.

I5!. Raphanus Raphanistrum, Linn. [Lit. Brit. 23 ; N.C.E. 1, 3 b, $11,14,18,32,34,40$, Warnstorf, 2507.] Knuth's autumn observations at Kiel correspond rather closely with Müller's, making it to be visited in Germany by higher insects than at Clova.

Visitors. Lepidoptera. Rhopalocera: (I) Pieris brassicae L., I6. VI. 95. (2) P. napi L., I8. VI. 99. Hymenoptera. Aculeata: Apidae: (3) Bombus terrestris L., sh. 22. IX. 95. Diptera. Syrphidae: (4) Eristalis pertinax Scop., 22 IX. 95. (5) Ascia podagrica F., 22. IX. 95. Muscidae: (6) Pollenia rudis F., sh. I 7-22. IX. 95. Anthomyiidae: (7) Anthomyia sp., I7. IX. 95. Coleoptera. (8) Meligethes viridescens F., fp. and seeking h. I5-22. IX. 95 ; 18. VI. 99, very ab. All at $800 \mathrm{ft}$.

\section{Class B, $\S 30$. Syringa Type.}

152. Syringa vulgaris, Linn. [Lit. N.C.E. 1, 3 c, 14, 34.] In cultivation. Macroglossa bombyliformis shows a great preference for its flowers and was recorded as abundant on them in South Scotland in I899 (Entomologist, 1900, p. 45.)

Visitors. Lepidoptera. Rhopalocera: (I) Pieris napi L., sh. I2-I3. VI. 99. 
Heterocera: Sphingidae: (2) Macroglossa bombyliformis Esp., sh. a regular visitor, I3-I6. VI. 99. Noctuidae: (3) Habrostola tripartita Hufn., sh. I2-I5. VI. 99. (4) Xylocampa areola Esp., sh. I 2-I 5. VI. 99. (5) Anarta melanopa Thunb., sh. I 3. VI. 99. Diptera. Syrphidae: (6) Syrphus vitripennis Mg., I3. VI. 99. (7) Rhingia campestris Mg., sh. I3. VI. 99. Empidae: (8) Empis bilineata Lw., I3. VI, 99. Anthomyiidae: (9) Drymia hamata Deg., fp. I3. VI. 99. (10) Hylemyia nigrescens Rnd., I3. VI. 99. (I I) Anthomyia sulciventris Ztt., fp. I3-I 4. VI. 99. Coleoptera. (I 2) Meligethes viridescens F., fairly ab. fp. I0-I 2. VI. 99. All at 8-900 ft.

\section{Class B, $\S 3 \mathrm{I}$. Mentha Type.}

153. Thymus Serpyllum, Linn. [Lit. Brit. 23, Marquand 1513; N.C.E. 1, 3 c, 12, 14, 14 a, 16, 18, 30, 32, 34, 40 ; Arct. 38 ; Alps 2, 16, 34; Pyren. 17.] Gynodioecious. It is well visited by high types of insects.

Visitors. Lepidoptera. Rhopalocera: (I) Argynnis aglaia L., sh. 25 . VI.-4. VII. 95 ; 27 . VI.-I. VII. 96, 8-I,700 ft. (2) A. selene Schiff., sh. 27 . VI. 95, I,000 ft. (3) Coenonympha pamphilus L., sh. 20. VI-6. VII. 95; 2. VII. $96 ; 8-\mathrm{I}, 800 \mathrm{ft}$. (4) Polyommatus phloeas L., 25. VI. 95, $800 \mathrm{ft.}$ (5) Vanessa urticae L., sh. 6-8. VII. 95; 6-IO. VII. 96, 800 ft. (6) Lycaena icarus Rott., sh. 6. VII. 95 ; 27. VI.-10. VII. 96, 8-1,000 ft. (7) Erebia epiphron Kn., sh. 25. VI. 95, I 8-2,000 ft. (8) Pieris napi L., I 9. VI. 96, 800 ft. Heterocera: Geometridae: (9) Xanthorhoe salicata Hüb., sh. 2 5. VI. 95 ; 24. VI. 96, 8-900 ft. (10) Psodos trepidaria Tr., sh. 25. VI. 95, I0--I,500 ft. Crambidae: (I I) Pyrausta alpinalis Schiff., sh. 5. VII. 95, $800 \mathrm{ft}$. Tineidae: (I2) Glyphipteryx fuscoviridella Haw., sh. I. VII. 95, $800 \mathrm{ft}$. Pterophoridae: (I3) Pterophorus tetradactylus L., sh. 28. VI. 95, I,500 ft. Hymenoptera. Aculeata: Apidae: (I 4 ) Apis mellifica L., sh. I I. VII. 96, $800 \mathrm{ft}$. (I 5 ) Bombus terrestris L., sh. 25 . VI.-6. VII. 95, $800 \mathrm{ft}$. (I 6) B. lapponicus F., sh. 25. VI.-2. VII. 95; 22. VI.-IO. VII. 96, 8-2,300 ft. (I 7) B. pratorum L., sh. 26. VI.-r. VII. 95, 8-900 ft. (18) B. agrorum F., 26. VI. 95, $800 \mathrm{ft}$. Vespidae: (19) Vespa norvegica F., sh. 23. VII. 95, 800 ft. Petiolata tubulifera: Chrysididae: (20) Odynerus pictus Curt., sh. 25. VI. 95, $800 \mathrm{ft}$. Petiolata parasitica: Ichneumonidae: (2 I) Alomyia debellator (Fabr.)? I. VII. 95, 800 ft. Diptera. Syrphidae: (22) Platychirus manicatus Mg., sh. 25. VI.-5. VII, 95 ; 35. VI. 96, 7-800 ft. (23) Melanostoma mellinum L., 25 . VI. 95, $800 \mathrm{ft}$. (24) Sericomyia lappona L., sh. I-4. VII. 95 ; 6. VII. 96, 8-I,700 ft. (25) Volucella bombylans L., sh. 5 . VII. $95,800 \mathrm{ft}$. (26) Eristalis arbustorum L., sh. I-6. VII. 95, 800 ft. (27) Syritta pipiens L., fp. 23. VII. 95, $800 \mathrm{ft}$. Empidae: (28) Empis tessellata F., sh. 4. VII. 94 ; 25. VI.-6. VII. 95; 8-I,000 ft. Sarcophagidae: (29) Cynomyia mortuorum L., sh. II. VII. 96, $800 \mathrm{ft}$. Muscidae: (30) Lucilia cornicina F., sh. and fp. 2 I. VI.-5. VII. 95 ; го. VII. $96,800 \mathrm{ft}$. (3 I) Calliphora erythrocephala Mg., sh. 8-I 7. VII. 95 ; 25 . VI.-I I. VII, 96, 8-I,000 ft. (32) C. vomitoria L., sh. I-2 r. VII. 95, 8-900 ft. (33) Pollenia rudis F., sh. 25. VI. 96, $700 \mathrm{ft}$. Anthomyiidae: (34) Hyetodesia incana W. ? sh. $22-25$. VI. 95 ; 2. VII. $96,8-$ I, 300 ft. (35 and 36$)$ H. spp., sh. 4. VII. 94 ; 25. VI. 95 ; 4. VII. 95, 18-2,000 ft. (37) Coenosia sp., I. VII. 95, $800 \mathrm{ft.}$ (38) Spilogaster quadrum F., fp. 23. VII. 95, $800 \mathrm{ft.}$ 
I54. Mentha rotundifolia, Linn. [Lit. N.C.E. 21 b.] An escape from cultivation.

Visitors. Hymenoptera. Aculeata: Apidae: (I) Bombus terrestris L., ? sh. Diptera. Sarcophagidae: (2) Cynomyia mortuorum L., sh. Muscidae: (3) Lucilia cornicina F., sh. (4) Calliphora erythrocephala Mg. (5) Pollenia rudis F., fp. Cordyluridae. (6) Scatophaga stercoraria L., fp. Coleoptera. (7) Meligethes viridescens F. All 20. IX. 95, $900 \mathrm{ft}$.

\section{Class $\mathrm{B}, \S 32$. Linnaea Type.}

155. Linnaea borealis, Linn. [Lit. Brit. 23, 39 ; N.C.E. 1, 3 c, 8, 14, $14 \mathrm{a}, 16,18,31,34$; Arct. 36 ; Alps 2, 9.] The mouth of the bell is $5 \mathrm{~mm}$. in diameter, the length of the bell is $8 \mathrm{~mm}$. The stigma stands $\mathrm{I} \mathrm{mm}$. beyond the longer stamens and is curved downwards so as to rub on the back of a visiting insect struggling to penetrate the forest of hairs which guard the honey.

Visitors. Diptera. Anthomyiidae: (I) Trichophthicus hirsuiulus $Z \mathrm{tt}$., fp. Coleoptera. (2) Meligethes viridescens F. Both at I,800 ft. 6. VII. 96.

\section{Class B, $\S 33$. Habenaria viridis Type.}

I56. Habenaria viridis, R. Br. [Lit. Brit. Darwin 483; Alps 2.]

Visitor. Coleoptera. (I) Anthophagus alpinus Payk., sh. I. VII. 96, $2,400 \mathrm{ft}$

\section{Class B, $\S 34$. Calluna Type.}

I57. Calluna vulgatis, Salisb. [Lit. Brit. 23, 34, $39 ;$ N.C.E. $1,3 \mathrm{c}$, 4, 9, 11, 14, 14 a, 16, 18, 24, 25, 33, 34, 40 ; Arct. 36 ; Alps 2 ; Pyren. 17.] The first heath to flower is generally on some wind-swept point high up, where the plant lies twisted close to the ground ; then it bursts into blossom all over the district. As we have shown (Lit. 39) it is probably somewhat anemophilous. We have found plants at Clova upwards of thirty years old (Graebner, in Engler's Bot. Jahrbuicher, xx. 1895, p. 500, says that it ceases to flower after fifteen years and dies out in about twenty-four years).

Visitors. Lepidoptera. Rhopalocera: (I) Lycaena icarus Rott., sh. 22 VII. 95, $800 \mathrm{ft}$. Heterocera: Noctuidae: (2) Hydroecia nictitans Bkh., sh. I7. IX. 95, $700 \mathrm{ft}$. Hymenoptera. Aculeata: Apidae: (3) Bombus terrestris L., sh. 14-23. IX. $95,7-2,900 \mathrm{ft}$. (4) B. lapponicus F., sh. 30. VI.-8. VII. 96, 20-2,200 ft. (5) B. scrimshiranus Kirby, 14. IX. 95, 2,000 ft. Formicidae: (6) Formica fusca Latr., sh. I 7. IX. 95, $700 \mathrm{ft}$. Petiolata parasitica : Ichneumonidae: (7) I sp., sh. I9. IX. 95, 2,400 ft. Diptera. Syrphidae: (8) Platychirus sp., sh. 22. VI. 95, I,000 ft. Empidae: (9) Empis ? vernalis Mg., sh. I9. IX. 95, 2,400 ft. Muscidae: (I0) Lucilia cornicina F., sh. and fp. I 4-I 9. IX. 95, 20-2,400 ft. (I I) Pollenia rudis F., sh. and fp. I 4-2 I. IX. 95, 9-2,400 ft. Anthomyiidae: (I 2) Hyetodesia variabilis Fln. 30 . VI. 
96, 2,300 ft. (I 3 ) Trichophthicus sp., fp. 8. VII. 96, I,400 ft. (I 4) Anthomyia sp., sh. 2I. IX. 95, I,100 ft. Cordyluridae: (I5) Scatophaga stercoraria L., sh. 20-2 I. IX. 95, I I-2,500 ft. Coleoptera. (I6) Meligethes viridiscens F., fp. I 7-2 I. IX. 95, 7-1,000 ft. Thysanoptera. (I 7) Thrips sp., I 9. IX. 95, 2,400 ft.

\section{Class $\mathrm{B}, \S 35$. VAlerian TyPe.}

158. Valeriana officinalis, Linn. [Lit. Brit. 23 ; N.C.E. 1,3 c, 18, 32, 33, 34; Arct. $36 ;$ Alps, 2, 9, $16 ;$ Pyren. 17.] We have referred this flower to Class B, not following those who place it in $B^{\prime}$. In Germany, Flanders, and the Pyrenees it has been shown to be rather a Syrphid flower. In the Alps butterflies visit it freely.

Visitors. Lepidoptera. Rhopalocera: (1) Pieris brassicae L., sh. 22. VII. 95, $800 \mathrm{ft}$. Heterocera: Noctuidae: (2) Dianthecia cucubali Fues., sh. 26. VI. 96, $800 \mathrm{ft}$. (3) Noctua brunnea Schiff., 23. VII. 95, $800 \mathrm{ft}$. (4) N. baja F., 22 . VI. 95, 800 ft. (5) N. C-nigrum L., sh. 2. VI. 95, $800 \mathrm{ft}$. (6) Celaena haworthii Cuc., sh. 6. VII. $95,800 \mathrm{ft}$. (7) Charaeas graminis L., sh. 20-23. VII. 95, $800 \mathrm{ft}$. Geometridae: (8) Thera variata Schiff,, sh. 6. VII. 95, 800 It. Crambidae: (9) Pyrausta alpinalis Schiff., sh. 6. VII. 95, 800 ft. Hymenoptera. Aculeata: Apidae: (ro) Apis mellifica L., sh. I5-22. VII. 95 ; 3-Ir. VII. $96,800 \mathrm{ft}$. (I I) Bombus pratorum L., sh. I 2-23. VII. 95, 7-800 ft. (I 2) B. terrestris L., sh. Ir. VII. 96, $800 \mathrm{ft}$. Sessiliventres: Tenthredinidae: (13) Allantus arcuatus Forst., eating petals 10. VII. 95, $800 \mathrm{ft}$. Petiolata parasitica : Ichneumonidae: (14) Polyrrhembia tenebricosa Grav., 2. VII. 95, $800 \mathrm{ft}$. (I 5) I sp., 3. VII. 96, $800 \mathrm{ft}$. Diptera. Syrphidae: (I6) Leucozonia lucorum L., sh. I 5. VII. 95, $800 \mathrm{ft}$. (17) Platychirus manicatus Mg., sh. and fp. 6-20. VII. 95 ; 3-Ir. VII. 96, 800 ft. (I 8 ) P. peltatus Mg., sh. 22. VII. 95, $800 \mathrm{ft}$. (19) Syrphus compositarum Verrall, sh. I7. VII. 95, $800 \mathrm{ft} . \quad$ (20) S. ribesii L., fp. I I-20. VII. 95, $800 \mathrm{ft}$. (2I) S. ? balteatus Deg., sh. I5. VII. 95, $800 \mathrm{ft}$. (22) Volucella bombylans L., sh. 10-2 2. VII. 95, $800 \mathrm{ft}$. (23) Eristalis arbustorum L., sh. 4-22. VII. 95; 24 . VI.-II. VII. 96, $800 \mathrm{ft}$. (24) E. rupium sh. and fp. 2-2 2. VII. 95, $800 \mathrm{ft}$. (25) Heliophilus pendulus L., sh. I I. VII. 96, $800 \mathrm{ft}$. (26) Syritta pipiens L., sh. I7. VII. 95, $800 \mathrm{ft}$. Empidae: (27) Empis tessellata F., sh. 4-20. VII. 95 ; 24. VI.-II. VII. 96, 800 ft. (28) E. bilineata Lw., 30. VI. 95, $700 \mathrm{ft}$. (29) E. punctata Mg., sh. I7. VII. 95, $800 \mathrm{ft}$. Tipulidae: (30) Tipula paludosa Mg., 22. VII. 95, 800 ft. Tachinidae: (3I) Siphona geniculata Deg., sh. 1o. VII. 95, $800 \mathrm{ft}$. Muscidae: (32) Calliphora vomitoria L., sh. and fp., I 7-22. VII. 95, 800 ft. (33) C. erythrocephala Mg., sh. and fp. 20. VII. 95 ; i r. VII. 96 , $800 \mathrm{ft}$. (34) Pollenia rudis F., sh. and fp. 4-I2. VII. 95, 7-800 ft. Anthomyiidae: (35) Hyetodesia incana W., sh. and fp. 2-I 7. VII. 95 ; 24. VI. 96, 800 lt. (36) H. basalis Ztt., sh. and fp. 2-4. VII. 95, $800 \mathrm{ft}$. (37) Spilogaster nigrivenis Ztt., fp. 3 . VII. $96,800 \mathrm{ft} .\left(3^{8}\right)$ Trichophthicus sp., sh. and fp. I 7. VII.-95; 24. VI. 96, $800 \mathrm{ft}$. Cordyluridae: (39) Scatophaga stercoraria L., sh. and fp. 20-22. VII. 95, $800 \mathrm{ft}$. Coleoptera : (40) Meligethes viridescens F., sh. 2. VII. 95 ; 20. VI.-6. VII. 96, 7-I,700 ft. (4I) Epuraea aestiva L., sh. 2-6. VII. 95, 800 ft. Trichoptera : (42) I sp., 22. VII. 95, $800 \mathrm{ft}$. 


\section{Class $B, \S 36$. Polygonim viviparum Type.}

159. Polygonum viviparum, Linn. [Lit. Arct. 7, 34, 36, 38; Alps 2, $9,16,21$ a, 21 b.] Reproduction is very largely by bulbils.

Visitors. Lepidoptera. Heterocera: Noctuidae: (I) Eupithecia nanata Hb., ? sh. 27. VI. 95, $800 \mathrm{ft}$. Crambidae: (2) Pyrausta alpinalis Schiff., 4. VII. 95, 2,700 ft. Tortricidae: (3) Tortrix sp., sh. 5. VII. 95, 800 ft. (4) Gelechia sp., sh. 24. VI. 95, $800 \mathrm{ft}$. Hymenoptera. Sessiliventres. Tenthredinidae: (5) Allantus arcuatus Forst., I. VII. 95, 800 ft. Diptera. Syrphidae: (6) Platychirus manicatus Mg., 4. VII. 95, $900 \mathrm{ft}$. (7) Syrphus tricinctus Fln., sh. 27. VI. 95, $800 \mathrm{ft} . \quad$ (8) Eristalis rupium F., sh. 27. VI. 95, $800 \mathrm{ft}$. (9) Syritta pipiens L., sh. I 7. VI. 95, $800 \mathrm{ft}$. Empidae: (10) Empis tessellata F., 4. VII. 94, $800 \mathrm{ft}$. Bibionidae: (II) Dilophus albipennis Mg., 10. VII. 96, 2,000 ft. Muscidae: (12) Pollenia rudis F., 4. VII. 94, $800 \mathrm{ft}$. Anthomyiidae: (r 3 ) Hyetodesia incana W., sh. 27. VI.-4. VII. 95, 8-I,800 ft. (14) H. lucorum Fln., 29. VI. 95, $800 \mathrm{ft}$. (I5) H. basalis Ztt., 29. VI. 95, $800 \mathrm{ft}$. (16) Spilogaster nigrivenis Ztt., fp. 2. VII. 96, 2,300 ft. (I 7) Drymia hamata Fln., sh. 4. VII. 95, I,800 ft. (I8) Hylemyia nigrescens Rnd., 24. VII. 95, $800 \mathrm{ft}$. (19) Homalomyia incisurata Ztt., 4. VII. 94, $800 \mathrm{ft}$. (20 and 2I) Anthomyia spp., sh. 22. VI. 95, I,700 ft. ; 6. VII. 96, 2,000 ft. Cordyluridae: (22) Scatophaga sp., sh. 4. VII. 95, I,800 ft. Sapromyzidae: (23) Lauxania cylindricornis F., 4. VII. 94, $800 \mathrm{ft}$. Hemiptera. (24) Nabis flavimarginatus, D. \& S., r. VII. 95, $900 \mathrm{ft}$.

\section{Class B, $\S 37$. Intermediate Rosaceous Type.}

16o. Prunus Padus, Linn. [Lit. N.C.E. 1, 3 b, 33 ; Alps 34.]

Visitors. Hymenoptera. Aculeata: Apidae: (I) Apis mellifica L., sh. 2 I-22. V. 97, 6-800 ft. Diptera. Syrphidae: (2) Syrphus punctulatus Verrall, sh. 2 I-22. V. 97, 6-800 ft. Anthomyiidae: (3) Anthomyia sulciventris Ztt., 2 I-22. V. 97, 6-800 ft. Coleoptera. (4) Meligethes ? viridescens F., 2 I. V. 97, $800 \mathrm{ft}$.

16r. Rubus Chamaemorus, Linn. [Lit. Brit. 23 ; N.C.E. 21 a, 21 b; Arct. 36, 38 ; Alps 34.] About 20 per cent. of the flowers get fully fertilized and set large fruits, the rest get fertilized in various degrees of completeness: frequently only one carpel may form a drupelet. Flies, which visit it, go by preference to the male flowers.

Visitors. Hymenoptera. Petiolata parasitica : Ichneumonidae: (I) Hemiteles? I6. VI. 95, 2,000 ft. Diptera. Syrphidae: (2) Melanostoma dubium Ztt., I 9. VI. 95, 2,000 ft. (3) Syrphus vitripennis Mg., I3. VI. 99, 2,300 ft. Empidae: (4) Empis lucida Ztt., sh. I6. VI. 95 ; I 7. VI. 99, 9-2,800 ft. (5) E. vernalis Mg., I 7. VI. 99, 2,800 ft. (6 and 7) E. spp., sh. I I-I 7. VI. 99 , I 5-2,800 ft. Bibionidae: (8) Scatopse sp., I I. VI. 99, I,500 ft. Anthomyiidae: (9) Hyetodesia incana W., I 9. VI. 95, 2,000 ft. (I0) Drymia hamata Fln., I9. VI. 95, 2,000 ft. (II) Hylemyia nigrescens Rnd., I I-I 2. VI. 99, I 5-2,500 ft. (I 2) Trichophthicus sp., I 6-I 9. VI. 95, I 8-2,000 ft. (13) Anthomyia radicum L., I 9. VI. 95 ; I6. VI. 99, 20-2,700 ft. (14) A. sulciventris Ztt., I 1. VI. 99, I,500 ft. (I 5) Azelia triquetra W., I3. VI. 99, 2,300 ft. Coleo- 
ptera. (I 6) Meligethes viridescens F., I6-I 9. VI. 95, 2,000 ft. ; 9-II. VI. 99, I 5-2,000 ft. (I 7) M. aeneus F., I 9. VI. 95, 2,000 ft. (I8) Anthobium torquatum Marsh., I9. VI. 95, 2,000 ft. (19) Anthophagus alpinus Payk., sh. 19. VI. 96, 3,000 ft. Thysanoptera. (20) Thrips sp., I I. VI. 99, I,500 ft.

162. Rubus suberectus, Anders. [Lit. Brit. 34, 39; Marquand 1513 ; N.C.E. 1,3 b $4,11,16,32,33,34,40$; Alps 16; Pyren. 17.] In R. suberectus we would include any or all the Rubi suberecti, were there more than one at Clova: for, following Sir Joseph Hooker, it is our endeavour, in Rubus, in Hieracia and elsewhere, to equalize our species and, as far as possible, neither to give a fictitious value to small variations nor to shirk the difficulty of drawing distinctions by lumping. But in the literature list above given we have quoted all citations of $R$. fruticosus, sensu amplissimo.

Visitors. Lepidoptera. Rhopalocera : (I) Pieris brassicae L., sh. 2 2. VI. 95. Heterocera : Eriocephalidae: (2) Eriocephala calthella L., sh. 30. VI. 95. Hymenoptera. Aculeata: Apidae: (3) Apis mellifica L., sh. 22-30. VI. 95. (4) Andrena analis Panz., sh. 30. VI. 95. Vespidae: (5) Vespa sylvestris Scop., sh. 30. VI. 95. Sessiliventres: Tenthredinidae: (6) Allantus arcuatus Forst., sh. 23. VI. 95. Diptera. Syrphidae: (7) Platychirus manicatus Mg., sh. 22. VI. 95; 22. VI. 96. (8) Syritta pipiens L., 22. VI. 95. Empidae: (9) Empis tessellata F., sh. 30. VI. 95. (10) E. punctata Mg., 22. VI. 96. Anthomyizdae: (II) Hyetodesia incana W., sh. 22-30. VI. 95 ; 22. VI. 96 . (12) Trichophthicus sp., sh. 30. VI.-5. VII. 95. Sepsidae: (13) Sepsis cynipsea L., sh. 22. VI. 96. Coleoptera. (I 4) Byturus tomentosus F., sh. and in cop. 30. VI. 95. Thysanoptera. (I5) Thrips sp., 30. VI. 95. All at $800 \mathrm{ft}$.

\section{Cotoneaster microphylla, Wall. In cultivation.}

Visitors. Hymenoptera. Aculeata: Vespidae: (I) Vespa norvegica F., sh. $\mathbf{x}$. VII. 96, $900 \mathrm{ft}$. freq. Diptera. Syrphidae: (2) Platychirus manicatus Mg:, I8. VI. 99, $900 \mathrm{ft}$. (3) Syrphus vitripennis Mg., I 5. VI. 99, $900 \mathrm{ft} . \quad$ (4) Heliophilus sp., I 5. VI. 99, $900 \mathrm{ft}$. (5) Syritta pipiens L., I 5. VI. 99, $900 \mathrm{ft}$. Tachinidae : (6) Siphona geniculata Deg., I8. VI. 99, $800 \mathrm{ft}$. Muscidae: (7) Calliphora erythrocephala Mg., 18. VI. 99, 900 ft. freq. Anthomyiidae: (8) Anthomyia sulciventris Ztt., 18. VI. 99, $800 \mathrm{ft}$.

164. Saxifraga oppositifolia, Linn. [Lit. N.C.E. 21 a ; Arct. 7, 34, $36,37 \mathrm{~b}, 38$; Alps 2, 21 b.] Protogynous, with self-pollination when the stamens dehisce. The brilliant patches of flowers in early April attract flies, but bees do not visit them. Plenty of fruit is ripened. The flowers of a handsome Pyreneean variety cultivated at Kew show no self-pollination.

Visitors. Diptera. Bibionidae: (1) Scatopse sp., 2 I. V. 97, I,700 ft. Muscidae: (2) Lucilia cornicina F., sh. I4. IV. 95, 10-1,200 ft. (3) Pollenia rudis F., sh. I 4. IX. 95, I0-I,200 ft. Anthomyizdae: (4) Anthomyia sulciventris Ztt., sh. and fp. 2 I. V. $97,2,300 \mathrm{ft}$. (5) A. sp., sh. and fp. 2 I. V. 97, I 7-2,200 ft. Helomyzidae: (6) Tephrochlamys sp., sh. 9. V. $98,2,300 \mathrm{ft}$. Sepsidae: (7) Sepsis ? cynipsea L., 
sh. I 4. IV. 95, 10-1,200 ft. Phoridae: (8) Phora sp., sh. 2 I. V. 97, 2,300 ft. Coleoptera. (9) Anthophagus alpinus Payk., sh. I. VII. 96, I,900 ft.

165. Ribes nigrum, Linn. [Lit. N.C.E. 1, 34; MacLeod, 1472.] In cultivation and as an escape from cultivation.

Visitors. Hymenoptera. Aculeata: Apidae: (I) Bombus terrestris L., sh. I3. V. 98. (2) B. lapponicus F., I6. V. 98. Both at $800 \mathrm{ft}$.

166. Menyanthes trifoliata, Linn. [Lit. Brit. $23 ;$ N.C.E. 1, 8, 9, 14, 18, 34, Warnstorf, 25007; Arct. 38.]

Visitors. Lepidoptera. Rhopalocera: (I) Pieris napi L. Diptera. Empidae: (2) Rhamphomyia sulcata Fln. Coleoptera. (3) Meligethes viridescens F., ? sh. All i 8. VI. $99,800 \mathrm{ft}$.

\section{Class B, $§ 38$. Large Geranium Type.}

167. Geranium sylvaticum, Linn. [Lit. Brit. ₹3, N.C.E. 3 b, 21 a, 21 b, 34; Arct. 34, 36; Alps 2, 21 a, 21 b.] The flowers sleep at night.

Visitors. Lepidoptera. Rhopalocera: (I) Pieris brassicae L., sh. 24. VI. 95, 900 ft. (2) Lycaena icarus Rott., sh. 24. VI. 95, 900 ft. Hymenoptera. Aculeata : Apidae: (3) Bombus terrestris L., sh. 5. VII. 95, $700 \mathrm{ft}$. (4) B. lapponicus F., sh. 24. VI. 95, $900 \mathrm{ft}$. and 6. VII. 96, 2,300 ft. (5) B. agrorum F., sh. 8. VII. 95, $800 \mathrm{ft}$, and 6. VII. 96, 2,c00 ft. (6) Andrena analis Panz., I I. VII. 96, $700 \mathrm{ft}$. (7) A. coitana Kirby, sh. 5. VII. 95, $700 \mathrm{ft}$. Petiolata parasitica: Chalcididae: (8) I sp., sh. I I. VII. 96, 800 ft. Diptera. Syrphidae: (9) Platychirus manicatus Mg., sh. 2-8. VII. 95; 25. VI.-I I. VII. 96, 7-800 ft. (Iо) P. peltatus Mg., sh. 22. VI. 96, 2,300 ft. (I I) P. clypeatus Mg., fp. 2. VII. 95, $800 \mathrm{ft}$. (I 2) Syrphus sp., sh. 25. VI. 95, 800 ft. (I3) Rhingia campestris Mg., sh. 24. VI.-8. VII. 95, 8-900 ft. (I 4) Eristalis arbustorum L., 25. VI. 96, $700 \mathrm{ft}$. Empidae: (I 5) Empis tessellata F., sh. 5. VII. 95; 29. VI. 96, 7-900 ft. (I6) E. punctata Mg., 5. VII. 95, $700 \mathrm{ft}$. (I 7) E. bilineata Lw., sh. I6. VI. 99, $800 \mathrm{ft}$. (I8) E. ? vernalis Mg。, 8. VII. 95, 800 ft. (I9) E. sp., sh. 29. VI. 96, $900 \mathrm{ft}$. Bibionidae: (20) Scatopse ?, 5. VII. 95, $700 \mathrm{ft}$. Muscidae: (2 I) Stomoxys calcitrans L., sh. 25. VI. 95, $800 \mathrm{ft}$. Anthomyiidae: (22) Hyetodesia incana W., sh. 2. VII. 96, 2,000 ft. (23) H. lucorum Fln., sh. 24. VI. 95, $900 \mathrm{ft}$. (24) H. basalis Ztt., sh. 24. VI. 95, $900 \mathrm{ft}$. (25) Limnophora solitaria Ztt., 26. VI. 96, 2,300 ft. (26) Drymia hamata Fln., sh. 26. VI.-2. VII. 96, 20-2,400 ft. (27) Hylemyia nigrescens Rnd., sh. I 5-1 9. VI. 99, 7-900 ft. (28) Anthomyia sp., sh. 4-5. VII. 95, 700 and 2,300 ft.

\section{Class B, $§$ 39. Veronica Type.}

168. Veronica Chamaeđrys, Linn. [Lit. Brit. 23, 34; N.C.E. 1, 3 c, $4,11,14,16,18,33,34,40$; Alps 2; Pyren. 17.]

Visitors. Lepidoptera. Heterocera: Eriocephalidae: (I) Eriocephala calthella L., 24. VI. 95, $800 \mathrm{ft}$. Hymenoptera. Aculeata: Apidae: (2) Andrena analis Panz., sh. 22-23. VI. 95, 8-900 ft. Petiolata parasitica: Chalcididae: (3) I sp., I5. 
VI.-2. VII. 95, 8-1,000 ft. Diptera. Syrphidae: (4) Platychirus manicatus Mg., sh. and fp. 20. VI.-20. VII. 95, 8-900 ft. fairly freq. (5) Chrysogaster ? hirtella, Loew, sh. 22. VI. 95, $800 \mathrm{ft}$. (6) Syrphus luniger Mg., sh. 23-26. VI. 95, 8-900 ft. (7) Ascia podagrica F., 20. VI. 95, $800 \mathrm{ft}$. (8) Rhingia campestris Mg., 28. VI. 95, $800 \mathrm{ft}$. Empidae: (9) Empis tessellata F., sh. 20. VII. 95, $800 \mathrm{ft}$. Tachinidae: (Iо) Siphona geniculata Deg., sh. 22. VI. 95 ; 22. VI. 96, 8-900 ft. Muscidae: (I I) Pollenia rudis F., 5. VII. 95, $700 \mathrm{ft}$. Anthomyizdae: (I2) Hyetodesia incana W., 23. VI. 95, $800 \mathrm{ft}$. (I3) Anthomyia sulciventris Ztt., sh. and fp. 25 . VI.-r 2. VII. 95; 27. V. 97, 7-800 ft. (г4) A. sp., fp. г2. VII. 95; го. VI. 99, 7-800 ft. (I5) Coenosia sp., sh. I-6. VII. 95, $800 \mathrm{ft}$. Opomyzidae: (r6) Opomyza germinationis L., 20. VI. 95; $800 \mathrm{ft}$.

169. Vercnica serpyllifolia, Linn. [Lit. Brit. $23 ;$ N.C.E. 1, 14, 34, Warnstorf 2507.] Abundant, but very little visited.

Visitor. Thysanoptera. (I) Thrips sp., I6. IX. 95, $800 \mathrm{ft}$.

I70. Veronica alpina, Linn. [Lit. N.C.E. 21 b ; Arct. 36, 37 c ; Alps $2,9,21 \mathrm{~b}$.] The flower is $6 \mathrm{~mm}$. broad; its tube $1.5 \mathrm{~mm}$. long. Ultimate self-pollination and apparently fertilization occurs. Abundance of seed is set.

Visitor. Diptera. (I) A small fly, sh. 22. VI. 95, 2,600 ft.

I7I. Veronica officinalis, Linn. [Lit. Brit. 23, 34; N.C.E. 1, 4, 14, 16, $18,30,34$; Alps $2,9,16,21$ a, $21 \mathrm{~b}, 34$.

Visitors. Hymenoptera. Aculeata: Apidae: (1) Andrena coitana Kirby, 26. VI. 95. Diptera. Syrphidae: (2) Platychirus manicatus Mg., 26. VI. 95. Anthomyiidae: (3) Hylemyia nigrescens Rnd., sh. 2 I. VI. 95. All at $800 \mathrm{ft}$.

172. Veronica scutellata, Linn. [Lit. N.C.E. 14.]

Visitors. Hymenoptera. Petiolata parasitica: Chalcididae: (I) I sp. sh. 6. VII. 95. Diptera. Empidae: (2) Clinoceira bipunctata Hal., 6. VII. 95. An!homyiidae: (3) Hyetodesia variabilis Fln., I. VII. 95. All at $800 \mathrm{ft}$. 34,40 .

I73. Veronica Beccatunga, Linn. [Lit. Brit. 23 ; N.C.E. 1, 3 c, 33,

Visitor. Diptera. Anthomyizdae: (1) I sp., I 7. VI. 9, $800 \mathrm{ft}$.

\section{Class B, $\S 40$. Myosotis Type.}

I74. Myosotis arvensis, Hoffm. [Lit. Brit. 23.]

Visitors. Diptera. Syrphidae: (I) Melanostoma mellinum L., sh. 22. IX. 95, $800 \mathrm{ft}$. Anthomyiidae: (2) Limnophora sp., sh. and fp. I. VII. 95; I I. VII. 96, 7-800 ft. Thysanoptera. (3) Thrips sp., I I. IX. 95, $800 \mathrm{ft}$. 18, 34.]

I75. Myosotis versicolor, Reichb. [Lit. Brit. $23 ;$ N.C.E. 1, 3 c,

Visitors. Diptera. Syrphidae: (I) Platychirus manicatus Mg., I 5. VI. 99. 
(2) P. albimanus F., I6. IX. 95. Tachinidae: (3) Siphona geniculata Deg., fp. I6. IX. 95. All at $800 \mathrm{ft}$.

I76. Myosotis repens, D. Don. [Lit. Brit. 23; N.C.E. 1, 14, 18, $30,33$.

Visitors. Diptera. Empidae: (I) Empis tessellata F., sh. I. VII. 95; 29. VI. 96, 8-I,300 ft. (2) E. stercorea L., sh. I. VII. 95, $800 \mathrm{ft}$. Dolichopodidae: (3) Dolichopus sp., fp. 29. VI. 96, I,800 ft. Anthomyiidae: (4) Hyetodesia incana W., sh. I. VII. 95, $800 \mathrm{ft}$. (5) H. variabilis Fln., I. VII. 95, $800 \mathrm{ft}$. (6) Limnophora sp., 6. VII. $95,800 \mathrm{ft}$. (7) Drymia hamata Fln., fp. 6. VII. 95, $800 \mathrm{ft}$. (8) Trichophthicus sp., sh. 29. VI.-6. VII. 96, 8-1,300 ft. Chloropidae: (9) Chlorops sp., 29. VI. 96, 1,300 ft. Coleoptera. (10) Meligethes viridescens F., fp. 29. VI. $96, \mathrm{I}, 300 \mathrm{ft}$.

\section{I77. Anchusa sempervirens, Linn. [Lit. N.C.E. 4.]}

Visitor. Hymenoptera. Aculeata: Apidae: (I) Bombus agrorum F., sh. going from flower to flower, ro. VI. 99, $500 \mathrm{ft}$.

\section{Class B, $\S_{4}$ I. Epilobium Type.}

I78. Epilobium alsinifolium, Vill. [Lit. N.C.E. 21 a ; Arct. 36;Alps 2,21 a.]

Visitor. Diptera. Ephydridae: ( I) Hydrellia griseola Fln., fp. 6. VI. 95, $900 \mathrm{ft}$.

I79. Epilobium montanum, Linn. [Lit. Brit. $23 ;$ N.C.E. 3 b; 9, 14, 18, 21 a ; Alps 34.]

Visitor. Diptera. Syrphidae: (I) Platychirus ? manicatus Mg., 5. VII. 96, I, $400 \mathrm{ft}$.

I8o. Epilobium angustifolium, Linn. [Lit. Brit. 23 ; N.C.E. 1, 3 b, 11, 16, 18, 25, 33, 34, 40;Arct. 36, 38;Alps 2, 9, 21 b, 34.] One of the Class B which most nearly is a Bee flower. Bombi visit it freely and Apis abundantly in most places. Our Clova observations want amplifying. In Arctic regions self-pollination occurs.

Visitors. Lepidoptera. Heterocera: Noctuidae: (I) Miana fasciuncula Haw., sh. 30. VI. 96. Hymenoptera. Aculeata: Apidae: (2) Bombus terrestris L., sh. 11. VII. 96. Diptera. Anthomyiidae: (3) Hyetodesia basalis Ztt., sh. 30. VI. 96. (4) Anthomyia radicum L., sh. II. VII. 96. (5 and 6) A. sp., sh. 2 I. VI.-I I. VII. 96. Sepsidae: (7) Sepsis cynipsea L., sh. I r. VII. 96. All at $800 \mathrm{ft}$. 
TABLE XXXIII.

Actual number of individuals visiting the flowers of Class B.

148. Cheiranthus Cheiri

I 49. Cardamine pratensis .

150. Arabis caucasica . .

I5I. Raphanus Raphanistrum

I52. Syringa vulgaris .

153. Thymus Serpyllum

I54. Mentha rotundifolia . . -

155. Linnaea borealis . . . -

156. Habenaria viridis. $: .:$

I58. Valeriana officinalis . 28

I59. Polygonum viviparum . -

160. Prunus Padus . . . 2

I6I. Rubus Chamaemorus . -

I62. Rubus suberectus . . . 9

163. Cotoneaster microphylla -

164. Saxifraga oppositifolia . -

165. Ribes nigrum . . . -

I66. Menyanthes trifoliata

167. Geranium sylvaticum.

168. Veronica Chamaedrys

169. Veronica serpyllifolia

I 7o. Veronica alpina . . .

I 7 I. Veronica officinalis . . -

I72. Veronica scutellata. . -

173. Veronica Beccabunga

I74. Myosotis arvensis. .

I75. Myosotis versicolor .

I 76. Myosotis repens .

I77. Anchusa sempervirens : -

178. Epilobium alsinifolium .

I79. Epilobium montanum .

I80. Epilobium angustifolium

\begin{tabular}{ll|l|l|l|l|l|l|l|} 
Total . . . . . & 44 & $\frac{69}{4.8}$ & $\frac{7}{\cdot 49}$ & $\frac{3}{\cdot 21}$ & $\frac{37}{2.59}$ & $\frac{5}{\cdot 35}$ & $\frac{8}{\cdot 56}$ & $\frac{99}{6.93}$ \\
\hline Percentage &. &. & 3.08 & $4 \cdot 83$ & 0
\end{tabular}

\begin{tabular}{|c|c|c|c|c|c|c|}
\hline $\begin{array}{l}\text { న } \\
\dot{8} \\
\text { ป }\end{array}$ & $\begin{array}{l}\dot{\dot{s}} \\
\dot{\vec{J}}\end{array}$ & $\approx$ & $\dot{\theta}$ & ن & 选 & ¿ัర \\
\hline - & - & - & - & - & - & I \\
\hline & 2 & 2 & 102 & 5 & - & II 7 \\
\hline- & - & - & 9 & & - & I 4 \\
\hline - & - & 2 & 5 & 163 & - & I 75 \\
\hline- & - & 5 & I 7 & 17 & - & 64 \\
\hline 3 & - & 24 & $9^{2}$ & - & - & 189 \\
\hline & - & - & I9 & 2 & - & 22 \\
\hline & - & - & 3 & I & - & 4 \\
\hline & - & - & - & 4 & $\overline{-}$ & $\begin{array}{r}4 \\
-8\end{array}$ \\
\hline & $\overline{-}$ & 76 & 21 & 2 & 1 & $\begin{array}{r}50 \\
26\end{array}$ \\
\hline & - & 5 & 29 & - & I & 43 \\
\hline & - & 3 & 2 & I & - & 8 \\
\hline & - & 28 & 59 & I I & 2 & IOI \\
\hline- & I & 6 & 18 & 8 & I & 47 \\
\hline & - & 4 & I I & - & - & $2 \mathrm{I}$ \\
\hline & - & - & I9 & I & - & 20 \\
\hline & - & - & - & - & - & 3 \\
\hline & - & - & I & 2 I & - & 24 \\
\hline & - & $4^{2}$ & $3^{I}$ & - & - & 85 \\
\hline & I & 20 & 35 & - & - & 88 \\
\hline & - & - & - & - & 1 & I \\
\hline & - & - & I & - & - & I \\
\hline & - & I & I & - & - & 3 \\
\hline & - & - & 2 & - & - & 3 \\
\hline & - & - & I & - & - & I \\
\hline & - & I & 2 & - & 3 & 6 \\
\hline & - & 2 & I & - & - & 3 \\
\hline & - & 5 & I 2 & I & - & I8 \\
\hline & - & - & $=$ & - & - & I \\
\hline & 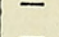 & - & 2 & 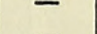 & - & 2 \\
\hline & I & 1 & 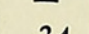 & 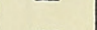 & - & 1 \\
\hline & & & & & & \\
\hline & 4 & 229 & ${ }^{6} 5^{6}$ & $24^{2}$ & I6 & 1429 \\
\hline & .29 & I 5.95 & $45 \cdot 9^{I}$ & I6.93 & $\mathrm{I} \cdot \mathrm{I} 2$ & \\
\hline
\end{tabular}

\section{TABLE XXXIV.}

Showing that Class B draws more than its share of distinctly desirable insects, and much more than its share of desirable insects.

\begin{tabular}{|c|c|c|c|c|}
\hline & \multicolumn{2}{|c|}{ Available. } & \multicolumn{2}{|c|}{ To Class B. } \\
\hline & No. & $\%$ & No. & $\%$ \\
\hline Distinctly desirable & $\mathbf{I}, 76_{3}$ & 10.19 & $2 \mathrm{I} 2$ & $14 \cdot 84$ \\
\hline Desirable . . & I, 277 & $7 \cdot 37$ & 246 & I 7.21 \\
\hline Indifferent & 12,993 & 75.08 & 910 & 63.68 \\
\hline Injurious . & $\mathrm{I}, 273$ & $7 \cdot 36$ & 6I & $4 \cdot 26$ \\
\hline
\end{tabular}


Class $B$ is found to attract rather more than its share of distinctly desirable insects (I 5: IO) and more of the desirable insects (I 7:7). To the distinctly desirable insects the Class proves relatively far less attractive than Class $\mathrm{H}$ (vide Table XXI in Part III) but relatively more attractive than Class B' (vide Table XI in Part II).

TABLE XXXV.

Insects visiting Class B in different seasons classed by Desirability.

\begin{tabular}{|c|c|c|c|c|c|c|}
\hline & \multicolumn{2}{|c|}{ Spring. } & \multicolumn{2}{|c|}{ Summer. } & \multicolumn{2}{|c|}{ Autumn. } \\
\hline Decidedly desirable & II & $7 \cdot 63$ & I 74 & 16.08 & 27 & 13.30 \\
\hline Desirable. • • & 5 & $3 \cdot 47$ & 236 & 21.81 & 5 & $2 \cdot 4 \mathrm{I}$ \\
\hline Indifferent . . & 127 & 88.19 & 623 & $57 \cdot 5^{8}$ & 160 & 78.81 \\
\hline Injurious . . . & I & .69 & 49 & 4.53 & I I & 5.41 \\
\hline Total. . . & I 44 & & 1082 & & 203 & \\
\hline
\end{tabular}

TABLE XXXVI.

Percentages of different groups of insects visiting Class B. in different seasons.

\begin{tabular}{|c|c|c|c|c|c|c|c|c|c|c|c|c|}
\hline & Apis. & Bomb. & $H m$. & $\begin{array}{l}\text { Phyt. } \\
\text { Entom. } \\
\text { Ants. }\end{array}$ & Wasps. & Lep.l. & Lep. $m$. & Lep.s. & $D m$. & Ds. & Col. & Etc. \\
\hline $\begin{array}{l}\text { Spring . } \\
\text { Summer } \\
\text { Autumn }\end{array}$ & $\begin{array}{l}4.16 \\
3.51\end{array}$ & $\begin{array}{r}2 \cdot 77 \\
3.60 \\
12.31\end{array}$ &.$\overline{64}$ & $\begin{array}{l}3 . \overline{60} \\
2 \cdot 95\end{array}$ & $\overline{73}$ & $\begin{array}{r}.69 \\
8.96 \\
.49\end{array}$ &.$\overline{92}$ & $\cdot-36$ & $\begin{array}{r}3 \cdot 47 \\
20.24 \\
2.46\end{array}$ & $\begin{array}{l}84.02 \\
45 \cdot 28 \\
22 \cdot 16\end{array}$ & $\begin{array}{r}4.16 \\
11.18 \\
56.65\end{array}$ & $\begin{array}{r}.69 \\
.92 \\
2.46\end{array}$ \\
\hline
\end{tabular}

TABLE XXXVII.

Desirability of visitors to Flowers of Class B according to colour in percentages.

\begin{tabular}{|c|c|c|c|c|c|}
\hline -. & Blue-lilac. & $\begin{array}{c}\text { Rose- } \\
\text { purple. }\end{array}$ & Iellow. & White. & Green. \\
\hline Decidedly desirable & IO. I7 & I9.86 & $3 \cdot 4 \mathrm{I}$ & $8 \cdot 45$ & $4^{2 \cdot} \cdot 86$ \\
\hline Desirable. • . & $20 \cdot 10$ & $29 \cdot 43$ & $\mathrm{I} \cdot \mathrm{I}_{4}$ & 23.00 & - \\
\hline Indifferent & 61.02 & $4^{8 \cdot 19}$ & $95 \cdot 45$ & $65 \cdot 26$ & $57 \cdot 12$ \\
\hline Injurious . . & $8 \cdot 7 \mathrm{I}$ & $2 \cdot 50$ & - & $3 \cdot 28$ & - \\
\hline Total no. of insects & $4^{\mathrm{I}} 3$ & 720 & 176 & 2I 3 & 7 \\
\hline
\end{tabular}

TABLE XXXVIII.

Percentages of different groups of insects visiting flowers of different colours in Class B.

\begin{tabular}{|c|c|c|c|c|c|c|c|c|c|c|c|c|}
\hline & $\frac{\widetilde{2}}{\nabla}$ & $\stackrel{2}{\cong}$ & $\stackrel{ \pm}{=}$ & 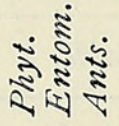 & है & ปें & $\begin{array}{l}\text { בे } \\
\text { ปें }\end{array}$ & نे & $\approx$ & 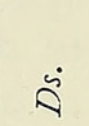 & $\overrightarrow{0}$ & 対 \\
\hline Blue- & & $2 \cdot 18$ & .97 & $7 \cdot 76$ & - & 8.00 & - & .72 & I 8.83 & $54 \cdot 36$ & 6.06 & $\cdot 97$ \\
\hline Rose-purple & 4.66 & 8.53 & $\cdot 3^{2}$ & I. 44 & I. I I & 9.82 & .64 & - & I $5.6 \mathrm{I}$ & $49 \cdot 9 \mathrm{I}$ & 4.83 & I. 28 \\
\hline Yellow & .63 & $I \cdot 76$ & - & - & - & $\mathrm{I} \cdot \mathrm{I} 4$ & - & - & $\mathrm{I} \cdot \mathrm{I} 4$ & 2.84 & $9^{2} \cdot 6 \mathrm{I}$ & - \\
\hline White. & 6.57 & $\cdot 4^{6}$ & $\cdot 4^{6}$ & $I \cdot 4^{\circ}$ & $\cdot 46$ & I. $40^{\circ}$ & $2 \cdot 8 \mathrm{I}$ & $\cdot 4^{6}$ & $19.7 \mathrm{i}$ & $55 \cdot 18$ & $9 \cdot 3^{8}$ & I. 87 \\
\hline Green . & - & $4^{2 \cdot 85}$ & - & - & - & - & - & - & - & - & $57 \cdot \mathrm{I} 4$ & - \\
\hline
\end{tabular}


Out of the whole available anthophilous fauna of (for the time of our observations) 17,306 individuals, 1,429 went to Class $B$. The species obtained attention as in Table XXXIII. In summer among the visitors were more long-tongued Lepidoptera than the class's share, and more Syrphidae (see Table XXXVI) ; in autumn among the visitors were more Bombi and more Coleoptera because Bombus went very freely to Thymus and Calluna, and Meligethes to Raphanus Raphanistrum. As shown in Table XXXIV, the summer visitors were of a higher type than the spring and autumn visitors.

In Tables XXXVII and XXXVIII the flowers of this class are divided according to their colour, and it is easily seen that the long-tongued Lepidoptera showed a distinct preference for flowers with a certain amount of blue in them, and the Bombi for rose-purple, and Apis for white and rosepurple. One yellow flower, viz. Raphanus Raphanistrum, drew so many individuals of Meligethes that the percentages for yellow are biased and the percentages for green are founded on too small figures for discussion of them to be possible.

We do not find Class $B$, by visitors, breaking into two halves as Class $\mathrm{B}^{\prime}$ did, yellow and white on one side, blue-lilac-purple and rose on the other, nor do we find the visitors visiting the colours as we found them visiting Classes $\mathrm{H}$ and $\mathrm{F}$.

Class $\mathrm{B}$ was found to attract nine species of the butterflies, sixteen large moths, six small moths including Eriocephala; of Hymenoptera, Apis, five species of Bombi, two of Andrena, two of Vespa, of Formica, of Odynerus, of Allantus, of four species of Parasitic Hymenoptera ; among Diptera, in the Syrphidae of Rhingia campestris, Volucella bombylans, Leucozonia lucorum, Helophilus, three species of Eristalis, one Chrysogaster, seven species of Syrphus, Syritta, Ascia, four species of Platychirus, and two of Melanostoma ; in Empidae of seven species of Empis including E. tessellata, of one Rhamphomyia and of one Clinoceira ; Siphona geniculata was the only Tachinid attracted; among Muscids the class attracted two Calliphorae, one Lucilia, one Pollenia and one Stomoxys, of Cynomyiidae one species, of Anthomyiids eighteen species including Drymia hamata, of Scatophagids one species, and of other orders of flies eleven species; of Coleoptera six species, one of Hemiptera, one of Trichoptera, and of Thrysanoptera one.

\section{Class Po. $\mathrm{B}, \S 42$. Pendant B-like Type.}

18I. Anemone nemorosa, Linn. [Lit. Brit. 23, 29 ; N.C.E. 1, 3 a, 4, 18, 33, 34, Warnstorf 2507.]

Visitors. Hymenoptera. Aculeata: Apidae: (I) Apis mellifica L., cp. I8. V. $97 ; 7-15$. V. 96, $800 \mathrm{ft}$. (2) Bombus lapponicus F., seeking h. and cp. 18-20. V. 97 ; 15 . V. 98,800 ft. Diptera. Syrphidae: (3) Platychirus discimanus Lw., fp. 18-20. V. 97 ; 7. V. $98,6-800$ ft. Mycetophilidae: (4) Sciara sp., 16. V. 98, 
700 ft. (5) Dilophus sp., I 8. V. 97, 800 ft. (6) Scatopse ?, 2 I. V. 97, I,600 ft. ? seeking h. 23. V. 96, 2,400 ft. Muscidae: (7) Lucilia cornicina F., 20. V. 97, $800 \mathrm{ft}$. Anthomyiidae: (8) Anthomyia sulciventris Ztt., fp. 20-22. V.97; 7-9. V. 98, 7-I,600 ft. (9) Anthomyia sp., 2 r. VI. 96 ; г 8-2 г. V. 97 ; г 5. V. 98 ; го. VI. 99, 7-1,500 ft. Cordyluridae: (Io) Scatophaga stercoraria L., fp. 21. V. 97. 7. V. 98; $8-2$, I $00 \mathrm{ft}$.

182. Pyrola media, Sw. [Lit. Alps 9.] All flowers set seed. Style not decurved as Kerner describes it.

Visitor. Hymenoptera. Petiolata parasitica: Chalcididae: (1) I sp. II. VII. $96,700 \mathrm{ft}$.

183. Pyrola rotundifolia, Linn. [Lit. N.C.E. 1, 4, 14, 24, 25, Warnstorf 2507; Arct. 36, 37 a ; Alps 2.] The style is strongly decurved and the stigma 4-8 $\mathrm{mm}$. from the anthers. All flowers set fruit. Protogyny very slight.

Visitors. Coleoptera. (I) Meligethes viridescens F., fp. Thysanoptera. (2) Thrips sp. Both 2. VII. 96, 2,000 ft.

TABLE XXXIX.

Individuals visiting the different flowers of Class B-like Po.

\begin{tabular}{|c|c|c|c|c|c|c|c|c|c|c|c|c|c|c|}
\hline Class Po $(B)$ & $\frac{\dot{\Sigma}}{\tilde{\nabla}}$ & ڤ్ & : & 辛 & 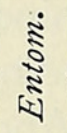 & $\frac{\dot{\nabla}}{\stackrel{\nabla}{\Sigma}}$ & $\begin{array}{c}3 \\
3 \\
3 \\
3\end{array}$ & $\begin{array}{l}\dot{2} \\
\dot{2} \\
\text { sú }\end{array}$ & 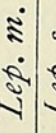 & $\stackrel{\Delta}{4}$ & $\dot{\theta}$ & i & 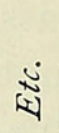 & 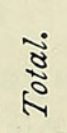 \\
\hline $\begin{array}{l}\text { 181. Anemone nemorosa } \\
\text { 182. Pyrola media } \\
\text { 183. Pyrola rotundifolia }\end{array}$ & $\frac{8}{-}$ & $\underline{4}$ & $\begin{array}{l}- \\
- \\
-\end{array}$ & $\begin{array}{l}- \\
-\end{array}$ & $\overline{\mathrm{I}}$ & $\begin{array}{l}- \\
-\end{array}$ & $\begin{array}{l}- \\
-\end{array}$ & $\begin{array}{l}- \\
-\end{array}$ & - & $\underline{3}$ & $\begin{array}{l}202 \\
-\end{array}$ & $\overline{-}$ & $\bar{z}$ & $\begin{array}{r}2 \mathrm{I} 7 \\
\mathrm{I} \\
4\end{array}$ \\
\hline Total. . . & 8 & 4 & - & - & I & - & - & - & - & 3 & 202 & 2 & 2 & 222 \\
\hline Percentage . . & $3 \cdot 60$ & $\mathrm{I} \cdot 80$ & - & - & $\cdot 45$ & - & - & - & - & $-\mathrm{I} \cdot 35$ & 90.99 & .90 & .90 & \\
\hline
\end{tabular}

Class AB, \& 43. Listera Type.

184. Listera cordata, R. Br. [Lit. Brit. Darwin 483; Alps 2.] The plant has a considerable amount of vegetative reproduction (see J. A. Z. Brundin in Bihang till K. Svenska Vetensk. Akad. Handlingar. XXI. 1875, No. I2). Self-pollination does not occur.

Visitors. Hymenoptera. Petiolata parasitica: Chalcididae: (I) I sp., I3. VI. 99, I,500 ft. Diptera. Tipulidae: (2) Limnophila ? luteolella Verrall, with pollinia adhering to its head and going from flower to flower, sh. I3. VI. 99, I,800 ft. Anthomyiidae: (3) I sp., ? seeking h. I9. VI. 96, I,500 ft. Coleoptera. (4) Malthodes sp., three individuals crawling from flower to flower, 27 . VI. $96,1,500 \mathrm{ft}$.

185. Malaxis paludosa, Sw. [Lit. Brit. Darwin 483; N.C.E. 14.] This little plant, only $6 \mathrm{~cm}$. high, is very easily overlooked. Its flowers are uniformly green except for the slightly different colours of the lines on 
the labellum. All the flowers of one raceme twist so as to face nearly in one direction. The only visitor seen, adhered to the pollinia, but did not pull them out.

Visitors. Hymenoptera. Petiolata parasitica: Chalcididae: (1) One sp., seeking h. 5. VII. $96,800 \mathrm{ft}$.

\section{Class AB, $\S 44$. Oxalis Type.}

186. Oxalis Acetosella, Linn. [Lit. Brit. 23. 29 ; N.C.E. 1, 11, 18, 34, 40 ; Alps 2.] In 96 per cent. of the flowers examined the stigmas and stamens were remote from one another, but in 4 per cent. they were near enough to make self-pollination by contact possible.

Visitors. Lepidoptera. Heterocera: Tineidae: (r) I sp., sh. I 5. VI. 99, I,900 ft. Diptera. Empidae: (2) Empis lucida Ztt., sh. I 2-I 5. VI. 99, 20-2,200 ft. (3) Empis sp., sh. 20. V. 97, I, 200 ft. Bibionidae: (4) Scatopse sp., sh. I 2. VI. 99, I,700 ft. Anthomyiidae: (5) Hylemyia nigrescens Rnd., sh. I2. VI. 99, 2,500 ft. (6) Anthomyia sp. sh. 23. V. $96 ; 20-2$ I. V. 97, I 2-2,600 ft.

187. Linum catharticum, Linn. [Lit. Brit. 23; N.C.E. 1, 14, 16, Warnstorf 2507; Pyren. 17.] The flowers remain closed in bad weather.

Visitors. Lepidoptera. Rhopalocera: (I) Coenonympha pamphilus L., 4. VII. 94, about 2,000 ft. Diptera. Tachinidae: (2) Siphona geniculata Deg., sh. 22. VI. 95, $800 \mathrm{ft}$. Anthomyiidae: (3) I sp., 10. VII. 96, 2,200 ft.

\section{Class AB, $\S 45$. Small Ribes Type.}

188. Ribes Grossularia, Linn. [Lit. Brit. 29, Marquand 1513; N.C.E. 1, 3 a, $21 \mathrm{~b}, 33,34,40$.] In cultivation and as an escape from it.

Visitors. Hymenoptera. Aculeata: Apidae: (I) Apis mellifica L., sh. I8-22. V. $97 ; 7-16$. V. 98, 7-800 ft. (2) Bombus terrestris L., sh. 19. V. 97, 8-900 ft. (3) B. lapponicus F., 20. V. 97, $800 \mathrm{ft}$. Vespidae: (4) Vespa ? sylvestris Scop., 20. V. $97,800 \mathrm{ft}$. Diptera. Empidae: (5) Empis lucida Ztt., sh. I3. V. 98, $800 \mathrm{ft}$. Bibionidae: (6) Dilophus sp., sh. and fp. 19-22. V. 97, 800 ft. Muscidae: (7) Lucilia cornicina F., I8. V. $97,800 \mathrm{ft}$. (8) Pollenia vespillo F., sh. 7 . V. $98,800 \mathrm{ft}$. Anthomyizdae: (9) Anthomyia sulciventris $\mathrm{Ztt}$., fp. 22. V. 97, $800 \mathrm{ft}$. Hemiptera. (10) Anthocoris sp., sh. I9. V. 97, $800 \mathrm{ft}$.

189. Ribes rubrum, Linn. [Lit. N.C.E. 1, 3 a, 16, 40;Alps 34.] In cultivation and as an escape from it.

Visitors. Diptera. Mycetophilidae: (I) Sciara sp., sh. 20. V. 97. Chironomidae: (2) Diamesa ? tonsa Hal., sh. I3. V. 98. (3) I sp., sh. 20. V. 97. Muscidae: (4) Lucilia cornicina F., sh. 18. V. 97. Anthomyizidae: (5) Anthomyia sulciventris Ztt., sh. and fp. 24. V. 97 ; 13. V. 98 . Thysanoptera. (6) Thrips sp., I8. V. 97. All at $800 \mathrm{ft}$. 
Class AB, $\S 46$. Berberis Type.

190. Berberis Aquifolium, Pursh. [Lit. N.C.E. 34.] In cultivation.

Visitors. Hymenoptera. Aculeata: Apidae: (I) Bombus terrestris L., sh. (2) B. lapponicus F., sh. Both I 4. V. 98.800 t.

\section{Class AB, § 47. ACer Type.}

191. Acer Pseudo-platanus, Linn. [Lit. N.C.E. 3 b, 14, 18, 21 b, 34, Warnstorf 2507.]

Visitors. Lepidoptera. Heterocera : Noctuidae: (I) I sp., I 5. VI. 99, $800 \mathrm{ft}$. Hymenoptera. Aculeata: Apidae: (2) Apis mellifica L., sh. 21-24. V. $96 ; 24$. V. 97, 7-800 ft. (3) Bombus terrestris L., sh. 2 r. V. 96; 19-26. V. 97, 7-800 ft. Vespidae: (4) Vespa sp., I 5. VI. 99, $900 \mathrm{ft}$. Petiolata parasitica: Ichneumonidae: (5) I sp. I5. VI. 99, $900 \mathrm{ft}$. Diptera. Bibionidae: (6) Bibio nigriventris Hal., I 5. VI. 99, $900 \mathrm{ft}$. Anthomyiidae: (7) Trichophthicus sp., sh. I5. VI. 99, 8-900 ft. Coleoptera. (8) Meligethes viridescens F., I 5. VI. 99, $900 \mathrm{ft}$. (9) Rhagonica limbata Thoms., I 5. VI. 99, $900 \mathrm{ft}$.

\section{Class AB, $\$ 48$. Caltha Type.}

192. Caltha palustris, Linn. [Lit. Brit. 23, 29, Darwin $485 ;$ N.C.E. 1, 3 a, 14, 16, 18, 34; Arct. 36;Alps 2, $21 \mathrm{~b} ;$ Pyren. 17.] Above I, 800 feet we get the variety minor; it flowers later than the type. As a result of counting the organs of twenty-eight flowers from 2,600 feet we found the stamens to average nearly 57 , and the carpels 4.5 (Cf. Burkill in Journ. Linn. Soc. Bot. xxxi. pp. 233-4).

Visitors. Hymenoptera. Petiolata parasitica: Chalcididae: (I and 2) 2 spp, 22. VI. $95,2,200 \mathrm{ft}$. and I5. V. $98,800 \mathrm{ft}$. Diptera. Syrphidae: (3) Chilosia fraterna Mg., sh. 24-26. VI. 95, 8-900 ft. Empidae: (4) Empis lucida Ztt., sh. I 2. VI. $99,2,500 \mathrm{ft}$. (5) E. ? borealis L., 27. V. $97,800 \mathrm{ft}$. (6) Rhamphomyia sulcata Fln., ? sh. 10. VI. 99, $700 \mathrm{ft}$. (7) Clinoceira stagnalis Hal., 19. VI. 95, 2,600 ft. Bibionidae: (8) Dilophus albipennis Mg., sh. 22. VI. 95, 2,400 ft. Chironomidae: (9) I sp., sh. I 5. V. 98, $800 \mathrm{ft}$. Tachinidae: (10) Siphona geniculata Deg., 2 I. V. 96, $800 \mathrm{ft}$. Muscidae: (I I) Pollenia vespillo F., ro-r 2. VI. 99, 700 and 2,500 ft. Anthomyiidae: (I 2) Hyetodesia incana W., I9. VI.-r. VII. $95 ; 23$. V. 96, 8-2,700 ft. (13) Trichophthicus sp., sh. 24. VI. 95, 2,000 ft. (14) Hylemyia nigrescens Rnd., fp. I 2. VI. 99, 2,500 ft. ab. (I5) Anthomyia sulciventris Ztt., I 9. VI. 95, 2,600 ft.; 22. V. $96,2,400 \mathrm{ft}$; $18-27$. V. $97,6-800 \mathrm{ft}$. (16 and I 7 ) Anthomyia spp. fp. and seeking h. I9-25. VI. $95 ; 22-23$. V. $96 ; 20$. V. $97 ;$ 13-I6. V. 98 ; 10-I 4. VI. 99, 7-2,600 ft. (I 8 ) Azelia aterrima Mg., 26. VI. 95, $800 \mathrm{ft}$. Phoridae: (I 9 ) Phora rufipes $\mathrm{Mg}$., fp. and seeking h. 22. V. 96, 2,400 ft. Coleoptera. (20) Meligethes viridescens F., sh. 22-24. VI. 95, I6-2,200 ft. (2 I) M. aeneus F., fp. and sh. 2 I. V. 96; 20. V. 97 ; 10. VI. 99, 7-900 ft. (22) Anthobium lapponicum Mann., fp. 2 r. V. 96,800 ft. Hemiptera. (23) Lygus ?, Io. VI. $99,700 \mathrm{ft}$. Neuroptera. 
(24) Chloroperla sp., I. VII. 95, $800 \mathrm{ft}$. (25) Nemoura sp., 22. VI. 95, 2,000 ft. Araneida. (26) Oligolophus sp. pushing itself amongst the stamens, 12. VI. 99, $700 \mathrm{ft}$. (27) A spider lying in wait, 20. VI. 97, 8-900 ft. twice.

\section{Class AB, $\S 49$. Oxycoccos Type.}

193. Vaccinium Oxycoccos, Linn. [Lit. Brit. 23 ; N.C.E. 1, 3 c, 14, 34, 35, Warnstorf 2507; Arct. 36, 37 b ; Alps 2, 9.]

Visitors. Hymenoptera. Petiolata parasitica: (I) I small sp. Diptera. Bibionidae: (2) Scatopse sp. Both seeking h. IO. VI. 99, $700 \mathrm{ft}$.

\section{Class AB, $\S 50$. Small Crucifer Type.} $33,34$.

194. Barbarea vulgaris, R. Br. [Lit. Brit. 23; N.C.E. $1,3 \mathrm{a}, 18$,

Visitor. Coleoptera. (I) Meligethes viridescens F., fp. and sh. on old and half-withered flowers, 10. VI. 99, $700 \mathrm{ft}$.

195. Cochlearia officinalis, Linn. [Lit. Brit. 23, 29 ; N.C.E. 1, 14, 25, 31, 35, Hildebrandt 1051.] There are two types (with intermediates) at Clova. The larger form, which grows in very wet places on the crags, has the anthers and stigma remote from one another by $\mathrm{I} \mathrm{mm}$. but on the same level: in the closing of the flower, on withering, self-pollination invariably occurs, fertilization resulting. In the smaller form, which is the plant from Little Kilrannoch, figured in the Journal of Botany, $\mathrm{xxx}$, Plate 326 , the anthers and stigma are only $\frac{1}{2} \mathrm{~mm}$. apart and the flower is smaller-the diameter from tip of petal to tip of petal being $6-8 \mathrm{~mm}$. Self-fertilization invariably occurs in the withering of the flower. It is noteworthy how variable are the flowers of this smaller type, five petals as figured in the Journal of Botany having been seen by us as well as six. Once seven stamens were found, the normally paired ones on one side being three in number.

Visitors. Lepidoptera. Heterocera: Geometridae:(I) Larentia salicata Hüb., sh. 22. VI. 95, 2,000 ft. Diptera. Mycetophilidae: (2) Sciara sp., I6. VI. 99, and ? sh. 22. VI. 95, 2,800 ft. Bibionidae: (3) Scatopse sp., sh. I6. VI. 99, 2,800 ft. Anthomyiidae: (4) Drymia hamata Fln., sh. I. VII. 96, 2,000 ft. (5) Trichophthicus sp., sh. 2. VII. 96, 2,800 ft. (6 and 7) Anthomyia spp., sh. 22. VI. 95 ; 16. VI. 99 , 25-2,800 ft. Coleoptera. (8) Anthophagus alpinus Payk., sh. I. VII. 96, 2,000 ft. (9) Ceuthorrhynchius contractus Marsh, sh. I. VII. 96, 2,000 ft. Neuroptera. (10) I sp., sh. I. VII. $96,2,200 \mathrm{ft}$.

196. Brassica Sinapis, Visiani. [Lit. N.C.E. 1, 3 b, 8, 11, 14, 18, 31, 32, 33, 34, 40 ; de Vries 2460.]

Visitors. Lepidoptera. Rhopalocera: (I) Pieris brassicae L., 22-30. VI. 95. 
Diptera. Empidae: (2) Empis tessellata F., sh. 30. VI. 95. Coleoptera. (3) Meligethes viridescens F., ? sh. 5. VII. 95. All at $800 \mathrm{ft}$.

197. Capsella Bursa-pastoris, Moench. [Lit. Brit. 23, 29, A. Bateson 151 ; N.C.E. 1, 3 b, 11, 14, 18, 25, 31, 33, 34, 40; Alps 34; Pyren. 17.]. Flowers were found to have been-all of them- $q$ in the early part of 1897 up to May 2oth, when some began to perfect stamens.

Visitors. Diptera. Tachinidae: (I) Siphona geniculata Deg., sh. I8. IX. 95 . Anthomyiidae: (2) I sp. 2I. VI. 96 . Both at $800 \mathrm{ft}$.

198. Iberis amara, Linn. [Lit. Brit. 23; N.C.E. 1, $40 ;$ Alps 9 ; Pyren. 17.] In cultivation.

Visitors. Lepidoptera. Rhopalocera: (I) Pieris napi L., sh. II. VI. 99. Heterocera. Geometridae: (2) Fidonia atomaria L., sh. II. VI. 99. Diptera. Syrphidae: (3) Syrphus vitripennis Mg., fp. and sh. II-I3. VI. 99. freq. (4) Eristalis arbustorum L., sh. II-I 2. VI. 99. (5) E. ? pertinax L., sh. 23. V. 97. Empidae: (6) Empis opaca F., I2. VI. 99, $800 \mathrm{ft}$. Tachinidae: (7) Siphona geniculata Deg., I I. VI. 99. Muscidae: (8) Lucilia sp. I3. VI. 99. Anthomyizidae: (9) Hylemyia nigrescens Rnd., I3. VI. 99. (I0) Anthomyia radicum L., I I-I 2. VI. 99. (I I) A. sulciventris Ztt., fp. 23. V. 97. Coleoptera. (I 2 ) Meligethes viridescens F., fp. Iо. VI. 99. (r3) Psylliodes napi Koch, fp. ro. VI. 99. All at $800 \mathrm{ft}$.

\section{Class $\mathrm{AB}, \S 5 \mathrm{I}$. Trollius Type.}

199. Trollius europaeus, Linn. [Lit.Brit.23 ; N.C.E. 1, 11, 21 b, 34 ; Arct. 7, Feilden and Geldart in Trans. Norfolk and Norwich Nat. Soc. VI. p. 162 ; Alps 2, 9,21 b.] Trollius is par excellence a shelter-flower. It closes in bad weather, and then there are generally many insects harbouring inside. Colonel Feilden used it in Russian Lapland as a means of collecting insects : ' when a sudden change took place, the globe-flower closed its petals into a tight ball but not before the flies, beetles, and many other kinds of insects had fled for refuge to its sheltering bowers'. At Clova self-fertilization may be common; setting of seed is invariable.

Visilors. Hymenoptera. Petiolata parasitica. Proctotrypidae: (I) I sp., I5. VI. 99, I,900 ft. Diptera. Syrphidae: (2) Chilosia sparsa Lw., 22. V. 97, $500 \mathrm{ft}$. Anthomyiidae: (3) Hyetodesia incana W., 20. VI. 95, 2,600 ft. (4) Drymia hamata Fln., 20. VI. 95, 2,100 ft. (5) Trichophthicus sp. 20. VI. $96,2,300 \mathrm{ft}$. (6) Anthomyia sulciventris Ztt., 22. V. 97 ; I5. VI. 99, 500 and I,900 ft. Coleoptera. (7) Meligethes aeneus F., 22. VII. 97 ; I 5. VI. $99 ; 500$ and I, 900 ft. (8) Anthophagus alpinus Payk., fp. 22. VI. 96, 2,400 ft. (9) Anthobium torquatum Marsh., 2 2. VI. 97, I5. VI. 99, 500 and I, $900 \mathrm{ft}$. All these insects were taken from the interior of flowers, where by reason of the structure of the perianth it is rarely possible to ascertain what they may be doing. 
Class AB, $\S 52$. Philadelphus Type.

200. Philadelphus coronarius, Linn. [Lit. N.C.E. 1,3 b, 18, 32, 34.] In cultivation.

Visitors. Lepidoptera. Heterocera: Nochidae: (I) Noctua?, sh. 2. VII. 96, $800 \mathrm{ft}$.

Class AB, § 53. Rhodiola Type.

20r. Sedum Rhodiola, DC. [Lit. Brit. 23 ; N.C.E. 21 b ; Arct. 7, 36, 38 ; Alps 2,34.] This alpine was found to be visited by flies in Novaya Zembya, and by flies and ants in the Alps.

Visitors. Hymenoptera. Petiolata parasitica. Proctotripidae: (I) I sp., I5. VI. 99, I,900 ft. Diptera. Syrphidae: (2) Platychirus manicatus Mg., fp. 23. V. 96, 2,400 ft. Empidae: (3) Empis lucida Ztt., 22. VI, 96, 2,400 ft. (4) Rhamphomyia sp., sh. 22. VI. 95, 25-2,600 ft. (5) Hilara canescens Ztt., fp. 23 . VI. 96, 2,400 ft. Bibionidae: (6) Scatopse sp., 15. VI. 99, I,900 ft. Anthomyiidae: (7) Hyetodesia incana W., 20. VI. 95 ; I 5. VI. 99, I 9-2,600 ft. (8) Hylemyia nigrescens Rnd., I 5. VI. 99, I,900 ft. (9) Anthomyia sulciventris Ztt., fp. 23. V. 96, 2,400 ft. (I0) Anthomyia sp., ? fp. 22. VI. 96 ; r 7 . VI. 99, 2,400 ft. Psilidae: (I I) Psila fimetaria L., sh. 22. VI. 95, 2,000 ft. Coleoptera. (I 2) Meligethes viridescens F., 20. VI. $96,2,400 \mathrm{ft}$. (I3) M. aeneus F., sh. 22. VI. 95 ; 15 . VI. 99 , 19-2,000 ft.

\section{Class AB, $\S 54$. Malus Type.}

202. Pyrus intermedia, Ehrh. Planted.

Visitors. Hymenoptera. Petiolata parasitica. Proctotrypidae: (I) I sp., sh. 19. VI. 99. Diptera. Empidae: (2) Empis tessellata F., sh. 18. VI. 99. (3) Rhamphomyia sulcata Fln., I8-I9. VI. 99. Bibionidae: (4) Bibio nigriventris Hal., I8. VI. 99. Muscidae: (5) Calliphora erythrocephala Mg., sh. 19. VI. 99. Anthomyizdae: (6) Anthomyia radicum L., sh. 19. VI. 99. (7) Anthomyiid., I8. VI. 99. Coleoptera. (8) Meligethes viridescens F., I8. VI. 99. (9) Phyllobius pyri L., I 8. VI. 99. All at $800 \mathrm{ft}$.

203. Pyrus Malus, Linn. [Lit. Brit. $34 ;$ N.C.E. 1, 40, Plateau, 2002 $N$. Am. Waite 2469.] In cultivation.

Visitors. Diptera. Empidae: (I) Rhamphomyia sulcata Fln., I2. VI. 99. Bibionidae: (2) Bibio nigriventris Hal., seeking h. 10. VI. 99. Simuliidae: (3) Simulium sp., sh. 10. VI. 99. Anthomyiidae: (4) Anthomyia sp., fp. I0-I 2. VI. 99. Sepsidae: (5) Sepsis sp., Io. VI. 99. Coleoptera: (6) Meligethes aeneus F., fp. ro-I2. VI. 99, abundant. All at $800 \mathrm{ft}$.

\section{Class AB, $\S 55$. Ranunculus Type.}

204. Ranunculus Auricomus, Linn. [Lit. Brit. 23, Winter in Journ. Bot. 1897 , p. 406 ; N.C.E. 1,3 a, 18, 33, 34, Warnstorf 2507; Arct. 36.] 
One observer-Jungner (in Bot. Notiser, 1894, p. 156 )-says that it is slightly anemophilous in Sweden.

Visitor. Diptera. Anthomyiidae: (I) Anthomyia sulciventris Ztt., 20. V. 97, $800 \mathrm{ft}$.

205. Ranunculus Flammula, Linn. [Lit. Brit. $23 ;$ N.C.E. 1,3 a, 14, $18,21 \mathrm{~b}, 25,34$.]

Visitors. Lepidoptera. Heterocera: Geometridae: (I) Larentia salicata Hb., sh. 10. VII. 96, I,500 ft. Eriocephalidae: (2) Eriocephala calthella L., 25. VI. 95, $900 \mathrm{ft}$. Diptera. Syrphidae: (3) Chilosia fraterna Mg., sh. 25-26. VI. 95; i 8. VI. 96, $800 \mathrm{ft}$. (4) Chrysogaster hirtella Lw., sh. r. VII. 95, $800 \mathrm{ft}$. Empidae: (5) Empis tessellata F., I. VII. 95, $800 \mathrm{ft}$. Bibionidae: (6) Dilophus albipennis Mg., sh. I. VII. 95, $900 \mathrm{ft}$. Anthomyïidae: (7) Hyetodesia incana W., 26. VI.-2. VII. 95 ; 18. VI. 96, $800 \mathrm{ft}$. (8) Spilogaster nigrivenis Ztt., I 9. VI. 96, I,500 ft. (9) Drymia hamata Fln., 2 r. VI. 95, 900 ft. (10) Hylemyia nigrescens Rnd., sh. 25. VI. 95 ; I 3 . VI. 99, $800 \mathrm{ft}$. (II) Trichophthicus sp., sh. and fp. 25 . VI.-6. VII. 95; I6. IX. 95, 8-I,200 ft. (I 2, I3, and I4) Anthomyia 3 spp., sh. and fp. 2 I. VI.-6. VII. 95 ; I3-2 I. IX. 95; 18. VI.-II. VII. 96, 7-2,300 ft. Sciomyzidae: (I5) Tetanocera ferruginea Fln., I. VII. 95, $800 \mathrm{ft}$. Coleoptera. (I6) Meligethes viridescens F., sh. and fp. 2 I-26. VI. 95 ; I 6. IX. 95 ; r6. VI. $96,7-900$ ft. ( ( 7 ) Donacia discolor Panz., sh. 21. VI.-6. VII. 95, 5-900 ft.

206. Ranunculus bulbosus, Linn. [Lit. Brit. 23, 34; N.C.E. 1,3 a, 14, 16, 21 b, 33, 34; Alps 2 ; Pyren. 17.]

Visitor. Diptera. Anthomyiidae: (I) Drymia hamata Fln., 20. V. 96, I, $400 \mathrm{ft}$.

207. Ranunculus acris, Linn. [Lit. Brit. 23; N.C.E. 1, 3 a, 11, 14, 14 a, 16, 18, 21 b, 25, 30, 32, 40; Arct. 7, 36; Alps 2, 16; Pyren. 17.] No one has found such great variety of flies on this plant as we have. It is to be remarked that they are allotropous chiefly. This buttercup is very common and of wide range, and its flowers vary considerably in size; we have also found them with contabescent anthers. Müller observed eleven species of Lepidoptera on it in the Alps. The flowers hang in heavy rain, then serving as a shelter to Anthomyiids, e. g. Anthomyia sulciventris.

Visitors. Lepidoptera. Rhopalocera: (I) Pieris brassicae L., I 5. VI. 99, $800 \mathrm{ft} . \quad$ (2) Lycaena icarus Rott., sh. 26. VI. 95, $800 \mathrm{ft}$. Heterocera: Eriocephalidae: (3) Eriocephaia calthella L., sh. 24. VI.-3. VII. 95, 9-I,000 ft. Hymenoptera. Aculeata: Apidae: (4) Bombus terrestris L., sh. I5. VI. 99, $900 \mathrm{ft}$. (5) Andrena coitana Kirby, 5. VII. 95, $800 \mathrm{ft}$. Sessiliventres: Tenthredinidae: (6) Allantus arcuatus Forst., devouring the flower, I4. VI.-2 I. VII. 95 ; 29. VI.-6. VII. 96 ; 19. VI. 99, very freq. Petiolata parasitica: Ichneumonidae: (7) I sp., sh. 4. VII. 95; I I. VII. 96, $800 \mathrm{ft}$. Proctotrypidae: (8) Proctotrype ? sh. 2I. IX. 95, $800 \mathrm{ft}$. Chalcididae: (9 and Io) 2 spp., 27. VI.-6. VII. 95, 8-I,000 ft. (also i I) I sp., 26. VI. 95, 21-2,2c0 ft. Diptera. Syrphidae: (I 2) Chrysogaster hirtella Lw., sh. 8. 
VII. 95, $800 \mathrm{ft}$. (I3) Chilosia fraterna Mg., sh. 22-24. VI. 95 ; 18-29. VI. 96 ; I 5-I6. VI. 99, 8-I,500 ft. (I 4 ) Platychirus manicatus Mg., sh. I 7. VII. 95 ; 29. VI. 96 ; го. VI. $99,7-$ I,500 ft. (I 5 ) P. albimanus F., sh. and fp. 22-24. IX. 95, 8-I,000 ft. (I6) P. peltatus Mg., sh. 2 I. VI.-3. VII. 95, $900 \mathrm{ft}$. (I 7) P. clypeatus Mg., 29. VI.-2. VII. 95, 8-1,000 ft. (18) Melanostoma mellinum L., sh. and fp. I. VII. 95, I3-2 I. IX. 95, 7-800 ft. (19) Syrphus luniger Mg., I 5-16. VI. 99, $800 \mathrm{ft}$. (20) S. ? ribesii L. I6. VI.-3. VII. 96. 7-800 ft. (2 I) Eristalis arbustorum L., sh. 22. Vİ. 95, $800 \mathrm{ft}$. Empidae: (22) Empis punctata Mg., sh. I5-20. VII. 95, $800 \mathrm{ft}$. (23) E. aestiva Lw., sh. 2 I. IX. 95, $800 \mathrm{ft}$. (24) Rhamphomyia spinipes Fln., ? sh. I6. IX. 95, 800 ft. (25) R. albosegmentata Ztt., sh. 22. VI. 95, I,400 ft. (26) Hilara matrona Hal., sh. I 7. VII. 95, $800 \mathrm{ft}$. Mycetophilidae : (27) Sciara sp., 20. VI. 95, I,700 ft. Bibionidae: (28) Dilophus albipennis Mg., sh. and fp. I5-I6. VI. 95, $900 \mathrm{ft}$., and 25. VI. 96, 2,200 ft. Tachinidae: (29) Siphona geniculata Deg., sh. 4. VII. 95 ; I 7 . IX. 95, 800 ft. Sarcophagidae: (30) Sarcophaga sp., Iо. VII. 96. $800 \mathrm{ft}$. Muscidae: (3 I) Lucilia cornicina F., sh. 24. IX. 95, I,600 ft. (32) Pollenia rudis F., fp. 22. IX. 95, 800 ft. Anthomyiidae: (33) Hyetodesia incana W., sh. I7. VI.-I 5. VII. 95 ; I6. IX. 95 ; I8. VI.-I I. VII. $96,8-2,500$ ft. ab. (34) H. lucorum Fln., 25 . VI. 95, I,000 ft. (35) H. basalis Ztt., sh. 20. VII. 95, $800 \mathrm{ft.} \quad\left(3^{6}\right) \mathrm{H} . \mathrm{sp}$., I 5-25. VI. 95, 8-I,400 ft. (37) Spilogaster nigrivenis Ztt., 27. VI. 96, I,400 ft. (38) Limnophora solitaria Ztt., 28. VI. 95, 2,200 ft. (39) Limnophora sp., sh. r. VII. 95 ; I9. VI. 96, I I-I, 200 ft. (40) Drymia hamata Fln., sh. and fp. 20 . VI.-Io. VII. 95 ; I6. IX. 95 ; 20. VI.-I. VII. $96,8-2,400$ ft. (4I) Trichophthicus hirsutulus Ztt., sh. 6. VII. 96, 2,000 ft. (42) Trichophthicus sp., sh. I6. VI.-I 5. VII. 95 ; 20. VI.-I. VII. 96, 8-2,400 ft. (43) Hylemyia variata Fln., sh. 21. VI. 95, $900 \mathrm{ft}$. (44) H. nigrescens Rnd., I3. VI. 99, $800 \mathrm{ft}$. (45) Anthomyia sulciventris Ztt., sh. 28. VI. $95 ; 27$. V. 97,700 and 2,200 ft. (46) A. radicum L., fp. I I-I6. VI. 99, 8-2,000 ft. (47 and 48) Anthomyia spp., sh. and fp. I5. VI.-22. VII. $95 ;$ r3-24. IX. 95 ; I6. VI.-Io. VII. 96 ; 10-I 9. VI. $99,7-2,600$ ft. (49) Homalomyia sp., sheltering I8. VI. 95, $900 \mathrm{ft}$. (50) Azelia aterrima Mg., 29. VI. 96, $900 \mathrm{ft}$. (5 I) Coenosia infantula Rnd., sh. 2 I. VI.-6. VII. 95, 8-I,700 ft. Cordyluridae: (52) Scatophaga stercoraria L., I 9. VI.-4. VII. 95; I 4-24. IX. 95, 8-2,700 ft. (53) S. maculipes Ztt., sh. 21. VI. 95, $900 \mathrm{ft}$. (54) S. squalida Mg., 4. VII. 95, 2,300 ft. Psilidae: (55) Psila sp., sh. I2. VII. 95, $700 \mathrm{ft}$. Sepsidae: $\left(5^{6}\right)$ Sepsis cynipsea L. sh. I 9. VI. 95 ; 25. VI. 96, 800 ft. Ephydridae: (57) Hydrellia griseola Fln., 4. VII. 95, $800 \mathrm{ft}$. Chloropidae: (58) Oscinis sp. 4. VII. 95 ; 26. VI. 96, 800 and 2,100 ft. Agromyzidae: (59) Agromyza sp., sh. I9. VI. 95, $800 \mathrm{ft}$. Coleoptera. (60) Meligethes viridescens F., sh. and fp. 19. VI.-4. VII. 95 ; I 7-24. IX. 95 ; I6-27. VI. 96 ; I 9. VI. $99,7-2,700 \mathrm{ft}$. ab. (6r) M. aeneus F., sh. 2r. VI.-6. VII. 95 ; 19. VI. 99, 8-I,600 ft. (62) Anthophagus alpinus Payk., sh. 4. VII. $95 ; 27$. VI.-2. VII. 96, 19-2,600 ft. (63) Anthobium minutum F., 24. VI. 95, I,500 ft. (64) A. lapponicum Mann., I. VII. 96, r,600 ft. (65) Tachyporus ? hypnorum F., 19. VI. 99, $800 \mathrm{ft}$. (66) Chrysomela staphylea L., devouring the flowers, 3. VII. 96, 2,500 ft. (67) Dascillus cervinus L., I-4. VII. 95, $800 \mathrm{ft}$. Hemiptera. (68) Acocephalus sp. 25. VI. 95, I,700 ft. (69) Leptopterna dolabrata L., 4 VII. $95,800 \mathrm{ft}$. Thysanoptera. (70) Thrips sp., I 5. IX. 95, $800 \mathrm{ft}$. 
208. Ranunculus repens, Linn. [Lit. Brit. 23 ; N.C.E. 1,3 a, 14, 16, $21 \mathrm{~b}, 25,31,34,40$; Arct. 7, 36; Alps 16, 21 a.] Freely visited by Anthomyiids.

Visitors. Hymenoptera. Sessiliventres: Tenthredinidae: (I) Allantus arcuatus Forst., lounging in flowers, I9. VI. 95, $800 \mathrm{ft}$. Diptera. Syrphidae: (2) I sp. I 4. VI. 95, $700 \mathrm{ft}$. Tachinidae: (3) Siphona cristata F., 22. VI. 95, $800 \mathrm{ft}$. Anthomyiidae: (4) Hyetodesia incana W., sh. $17-2$ 2. VI. 95 ; 18 . VI.-4. VII. 96, 800 ft. (5) Trichophthicus sp., fp. 20 . VI. 96, 2,300 ft. (6) Anthomyia sulciventris Ztt., sh. and fp. I 5. VI. $95 ; 27$. V. $97,7-800 \mathrm{ft}$. (7) Anthomyia sp., 22. VI. 95, $800 \mathrm{ft}$. Sepsidae:

(8) Sepsis cynipsea L., sh. 19. VI. 95, $800 \mathrm{ft}$. Coleoptera. (9) Meligethes viridescens F., sh. 29. VI. 96, $800 \mathrm{ft}$.

209. Ranunculus Ficaria, Linn. [Lit. Brit. 23, 29; N.C.E. 1,3 a, 3 b, $3 \mathrm{c}, 4,8,9,10,11,12,18,21 \mathrm{~b}, 33,34,40$, Warnstorf 2507, Hennings in Verhandl. bot. Ver. Brandenburg xxxvii., p. xxii, Delpino in Bull. Orto bot. Univ. Napoli, i. I 899 , p. 24.]

Visitors. Diptera. Muscidae: (I) Lucilia cornicina F., sh. 7. V. 98, $800 \mathrm{ft}$. Anthomyizdae: (2) Anthomyia sulciventris Ztt., 22. V. 97, $800 \mathrm{ft}$. (3) Anthomyia sp., sh. and fp. 20-22. V. 97 ; 16. V. 98, 6-900 ft. Araneida. (4) Xysticus sp., Jying in wait, 20 . V. $97,900 \mathrm{ft}$.

\section{Class AB, $\S 56$. Potentilla Type.}

210. Fragaria vesca, Linn. [Lit. Brit. $23 ;$ N.C.E. 1,3 b, 18, 21 b, 33, 34, 40; Alps 2; Pyren 17.]

Visitor. Diptera. Anthomyiïdae: I sp., 2. VII. 96, 2,100 ft.

21r. Potentilla Fragariastrum, Ehrh. [Lit. Brit. 29; N.C.E. 18; Pyren. 17.]

Visitors. Hymenoptera. Petiolata parasitica. (I) I sp. Diptera. Chironomidae: (2) I sp. Anthomyiïdae: (3) Anthomyia sp., sh. fairly freq. All. I4. V. 98, I, $400 \mathrm{ft}$.

212. Potentilla Tormentilla, Neck. [Lit. Brit. 23, 39 ; N.C.E. 1,3 b, 4, 14 a, 18, 21 a, 40; Alps 2 ; Pyren. 17.] The very abundance of this secured for it a great deal of observation and a consequently long list of visitors.

Visitors. Lepidoptera. Rhopalocera: (I) Coenonympha pamphilus L., sh. I 5-20. VI. 95 ; I. VII. 96, 9-I,400 ft. (2) Lycaena icarus Rott., sh. 25 . VI. 95 , $800 \mathrm{ft}$. Heterocera: Tineidae: (3) Glyphypteryx fuscoviridella Haw., 20. VI. 95, I,300 ft. Eriocephalidae: (4) Eriocephala calthella L., sh. and fp. 2-2 I. VII. 95, 8-I,700 ft. Hymenoptera. Aculeata: Apidae: (5) Bombus terrestris L., sh. I4. IX. 95, 800 ft. (6) Andrena analis Panz., sh. 6. VII. 95; 22. VI.-6. VII. 96, 9-I,500 ft. (7) A. coitana Kirby, sh. 6. VII. 95, $800 \mathrm{ft}$. Formicidae: (8) Formica fusca Latr., sh. 25 . VI.-1 7. VII. 95 ; 19-26. VI. $9^{6}$; I 5-1 7. VI. $99,8-2,100 \mathrm{ft}$. 
Myrmicidae: (9) Myrmica rubra L., sh. I6-23. VI. 95; 19. VI. 99, 8-900 ft. Petiolata parasitica: Ichneumonidae: (10) I sp., sh. 22. V.-3. VI. 95; 23. V. 97 , IO-I, $300 \mathrm{ft}$. Chalcididae: (I I and I 2 ) 2 spp., I 5-29. VI. 95 ; 29. VI. 96 ; I I. VI. 99, 8-2,500 ft. Diptera. Syrphidae: (г 3 ) Platychirus manicatus Mg., 2 I. VII. 95 ; 25 . VI. $96,7-1,000 \mathrm{ft}$. (I4) P. clypeatus Mg., 29. VI. 95, I,000 ft. (I5) P. albimanus F., 2 I. IX. 95, 800 ft. (I6) Melanostoma mellinum L., 20. VI. 95, r,000 ft. (I 7) Chilosia fraterna Mg., sh. 26. VI. 95 ; 19. VI. 96, 8-I,300 ft. (I 8) Chrysogaster hirtella Lw., sh. 22. VI. 95, 800 tt. (I9) Syrphus sp., 20. VI. 95, $900 \mathrm{ft}$. (20) Ascia ? podagrica F., 26. VI.-I. VII. 95, $800 \mathrm{ft}$. (21) Sphaerophoria scripta L., sh. 19. VI. 96, 2,200 ft. (22) S. nitidicollis Ztt., 20. VI. 95, I,600 ft. Empidae: (23) Empis lucida Ztt., I 2-I 4. VI. 99, I4-I,600 ft. Bibionidae: (24) Scatopse sp., 29. VI. 96, I,500 ft. Dolichopodidae: (25) Dolichopus atratus Mg., 22. VI. $96,800 \mathrm{ft}$. (26) D. rupestris Hal., sh. 6. VII. 95, I,500 ft. (27) Hercostomus nigripennis Fln., I. VII. 95, $800 \mathrm{ft}$. Tachinidae: (28) Siphona geniculata Deg., I8. VI. 99, 800 ft. Muscidae: (29) Calliphora erythrocephala Mg., 4. VII. 95 ; I6. VI. 99, 8-1,500 ft. Anthomyiidae: (30) Hyetodesia incana W., 26-29. VI. 95, 1,000 and 2,400 ft. (3I) Spilogaster nigrivenis Ztt., 30 . VI. 96, 2, I00 ft. (32) Limnophora sp., sh. I9. VI. 96, I 5-r,600 ft. (33) Drymia hamata F., 20. VI.-6. VII. $95 ; 20$. VI.-I. VII. 96, 8-2,500 ft. (34) Trichophthicus hirsutulus Ztt., 20. VI. 95 ; 6. VII. 96, I6-2,000 ft. (35) Hylemyia nigrescens Rnd., sh. 10-II. VI. 99, 7-I,300 ft. (36) Anthomyia radicum L., I0-18. VI. 99, 7-2,000 ft. (37) A. sulciventris Ztt., 20. VI. $95 ; 20-27$. V. $97,5-2,500$ ft. $(38$ and 39$)$ Anthomyia 2 spp., sh. and fp. 20. VI. 95 ; 14-2I. IX. 95 ; 22. V. 96 ; 19-22. VI. $96,8-2,500$ ft. (40) Coenosia sp., fp. 19-20. VI. 95 ; 27 . VI. 96 ; I I. VI. $99,8-2,000$ ft. Cordyluridae: (4I) Scatophaga sp., sh. 19. VI. 99, 2,200 ft. Ortalidae: (42) Pteropaectria frondescentiae L., sh. I-6. VII. 95, 9-1,700 ft. Ephydridae: (43) I sp. I 5. VI. 95, $900 \mathrm{ft}$. Chloropidae: (44) Oscinis sp., 20. VI 95, 8-1,200 ft. Phytomyzidae: (45) Phytomyza sp., I 4. IX. 95, I,300 ft. Phoridae: (46) Phora sp., 20. VI. 95 ; 20 . IX. 95, I0-I,300 ft. Coleoptera. (47) Meligethes viridescens F., sh. and fp. 24-29. VI. 95 ; 2 I. IX. 95 ; 19-25. VI. 96, 7-2,500 ft. (48) M. aeneus F., I. VII. 95, I,500 ft. Hemiptera. (49) I sp., I0. VI. 99 , $700 \mathrm{ft}$.

213. Potentilla maculata, Pourr. (P.aurea, Linn). [Lit. Brit. 23; Alps 2, $21 \mathrm{a}, 21 \mathrm{~b}$; Pyren. 17.] The flower is $18-20 \mathrm{~mm}$. across and very conspicuous. The mechanism seems to agree closely with that of $P$. Tormentilla. Honey is present in fair quantity.

Visitors. Diptera. Syrphidae: (I) Platychirus sp., I7. VI. 99, 2,300 ft. Anthomyiidae: (2) Drymia hamata Fln., seeking h. 20. VI. 96, 2,300 ft. (3) Anthomyia sulciventris Ztt., 25. VI. 96, 2,200 ft. Araneida. (4) Xysticus sp., lying in wait, I7. VI. $99,2,300 \mathrm{ft}$.

214. Potentilla Comarum, Nestl. [Lit. Brit. $23 ;$ N.C.E. 1, 8, 12, 14, $14 \mathrm{a}, 18,33 ;$ Arct. 34.] At the opening of the bud the stamens standing erect dehisce; then they move outwards and the anthers fall off. But it would seem that the outermost stigmas are receptive before all chance of pollination from the innermost stamens has passed away. The central 
stigmas are then left to be fertilized by some outside agent. The stamens are of three lengths, respectively 4,5 , and $6 \mathrm{~mm}$., the shortest are the innermost before the petals, the longest those before the sepals. Honey is abundant and protected by hairs.

Visitors. Hymenoptera. Aculeata: Apidae: (I) Andrena ? analis Panz., sh. 19. VI. 96. Petiolata parasitica. (2) I sp., sh. 27. VI. 96. Diptera. Empidae: (3) Empis bilineata Lw., 4. VII. 95. (4) E. stercorea L., sh. I. VII. 95. Chironomidae: (5) Ceratopogon sp., sh. I 8-27. VI. 96. Tipulidae: (6) Limnophila lineolella Verrall, sh. 5. VI. 95 ; 18. VI. 96. Anthomyiidae: (7) Hyetodesia incana W., sh. 28. VI.-I. VII. 95 ; 18. VI.-4. VII. 96, fairly freq. Coleoptera. (8) Meligethes viridescens F., sh. I8. VI. 96 . (9) M. aeneus F., sh. I. VII. 95. All at 800 ft.

\section{Class AB, $§ 57$. Parnassia Type.}

215. Parnassia palustris, Linn. [Lit. Brit. 23, 39; N.C.E. 1, 11, 14, 24, 25, 34, 35, 40; Arct. 36 ; Alps 2, 9; Pyren. 17.]

Visitors. Hymenoptera. Aculeata: Formicidae: (I) Formica fusca Latr., seeking h. I6. IX. 95, I2-I,300 ft. Myrmicidae: (2) Myrmica rubra L., sh. and seeking h. I6. IX. 95, I 2-I,300 ft. Petiolata parasitica. Ichneumonidae: (3) I sp., sh. I6. IX. 95, I,300 ft. Braconidae: (4) I sp., I6. IX. 95, I,300 ft. Diptera. Cecidomyiidae: (5) Cecidomyia sp., sh. I6. IX. 95, I,200 ft. Anthomyiïdae: (6) Hyetodesia incana W., fp. I6. IX. 95, I,200 ft. (7) Trichophthicus sp., I6. IX. 95, I,200 ft. (8) Anthomyia sp., I 3. IX. 95, $700 \mathrm{ft}$. Cordyluridae: (9) Scatophaga stercorea L., sh. I6. IX. 95, I,300 ft. Coleoptera. (Iо) Meligethes viridescens F., sh. I6. IX. 95, I 2-I,300 ft. (I I) Epuraea aestiva L., sh. I6. IX. 95, I, $200 \mathrm{ft}$. Thysanoptera. (I 2 ) Thrips sp., sh. I3-2 I. IX. 95, 7-I,000 ft.

\section{Class AB, $\S 58$. Intermediate Saxifraga Type.}

216. Saxifraga nivalis, Linn. [Lit. Arct. 36, 37 b.] It seems to be abundantly self-fertilized.

Visitors. Hymenoptera. Petiolata parasitica. (I) I sp. Io. VII. 96, $2,000 \mathrm{ft}$.

217. Saxifraga hypnoides, Linn. [Lit. Brit. 23.] It is fairly well visited by allotropous insects.

Visitors. Lepidoptera. Heterocera: Geometridae: (I) Psodos trepidaria Tr., sh. 22. VI. 95, $800 \mathrm{ft}$. Tortricidae. (2) I sp. 22. VI. 96, 2,500 ft. Hymenoptera. Petiolata parasitica. Ichneumonidae: $(3,4$, and 5$) 3$ sp., sh. I7-24. VI. $95 ; 26$. VI.-6. VII. 96, I6-2,500 ft. Cynipidae: (6) I sp., sh. 22. VI.-4. VII. 95, 18-27,000 ft. Sessiliventres. Tenthredinidae: (7) Nematus carinatus Htg., sh. I 7. VI. 99, 2,400 ft. Diptera. Syrphidae: (8) Platychirus manicatus Mg., sh. 22. VI. 95, I,800 ft. Empidae: (9) Empis tessellata F., sh. 22. VI. 95, 2,000 ft. (ro) E. lucida Ztt., 22. VI. 96, 23-2,400 ft. (I I) E. aestiva Lw., sh. 30. VI. 96, 2,100 ft. (I2 and I3) Empis spp., 22. VI. 95, 23-2,500 ft. Cecidomyïdae: (I4) Sciara sp., 
30. VI. 96, 2,100 ft. Bibionidae: (15) Dilophus albipennis Mg., sh. 22. VI. 95, I 8-2,300 ft. Chironomidae: (I 6) Tanypus nebulosus Mg., sh. I5. VII. 95, 2,400 ft. (17) Another Chironomid, sh. I7. VI. 95, I,800 ft. Limnobidae : (18) Dicranomyia morio F., sh. 22. VI.-3. VII. 96, 22-2,400 ft. Anthomyiidae: (19) Drymia hamata Fln., sh. 22. VI. 95 ; I. VII. 96, I 8-2,400 ft. (20) Trichophthicus hirsutulus Ztt., sh. 6-20. VII. 96, 23-2,400 ft. (2 I) Trichophthicus sp., sh. 20. VII. 96, 23-2,400 ft. (22) Anthomyia sulciventris Ztt., 22. VI. 95, I,800 ft. (23, 24, and 25) Anthomyia 3 spp., sh. 2 I. VI.-II. VII. 95 ; 22. VI.-6. VII. 96, I 5-2,600 ft. Cordyluridae: (26) Scatophaga stercoraria L., fp. 22. VI.-4. VII. 95; 30. VI. 96, $2 \mathrm{I}-2,500 \mathrm{ft}$. Phoridae: (27) Phora rufipes Mg., sh. 3. VII. 96, 2,200 ft. Coleoptera. (28) Meligethes viridescens F., sh. 22. VI. 95 ; 3-6. VII. 96, 18-2,400 ft. (29) Anthophagus alpinus Pâyk., sh. freq. 3-4. VII. 95; 22. VI.-6. VII. 96, 2 I-2,600 ft. (30) Helodes marginata F., 24. VI. 95, I,600 ft. (31) Epuraea aestiva L., sh. 3. VII. 96, 2,400 ft. Thysanoptera. (32) Thrips sp., sh. 3-4. VII. 95, 2,600 ft. Araneida. (33) Oligolophus sp., once in the flower, 20. VI. 96, 2,100 ft.

\section{Class AB, § 59. Alsine Type.}

218. Cerastium triviale, Link. [Lit. Brit. $23, \approx 9 ;$ N.C.E. $1,3 \mathrm{a}, 3 \mathrm{~b}$, $14,18,21 \mathrm{a}, 21 \mathrm{~b}, 25,34$.] Varieties from high up have larger flowers than occur low down. The stigmas of these ultimately recurve so far as to bring about self-pollination. The long stamens dehisce just before the short ones. ${ }^{1}$

Visitors. Hymenoptera. Petiolata parasitica. (I) sp., sh. 2. VII. 95, $800 \mathrm{ft}$. Diptera. Syrphidae: (2) Platychirus manicatus Mg., sh. and fp. 6. VII. 95 ; 25 . VI. 96, 7-800 ft. Mycetophilidae: (3) Sciara?, sh. 2. VII. 96, 2,800 ft. Chironomidae: (4) Orthocladius sp., sh. 29. VI. 95, I,600 ft. Anthomyiidae: (5) Limnophora sp., sh. I. VII. $95,800 \mathrm{ft}$. (6) Anthomyia radicum L., I O-I I. VI. 99, 7-800 ft. (7) Trichophthicus sp., 2. VII. 96, 2,800 ft. Phoridae: (8) Phora sp., I 3. VI. 99, $900 \mathrm{ft}$. Thysanoptera. (9) Thrips sp., sh. 3. VII. $95 ; 29$. VI. $96,8-1,000 \mathrm{ft}$.

219. Cerastium alpinum, Linn. [Lit. Brit. 23 ; Arct. 7, 34, 36, 37 a, 38 ; Alps 2, 9, $21 \mathrm{~b}$, Ludwig 1394.] The flower is conspicuous, the petals attaining a length of $\mathrm{I} 2 \mathrm{~mm}$. Fruit is set in plenty in July and later, but flowering continues beyond this.

Visitors. Hymenoptera. Petiolata parasitica. Chalcididae: (I) I sp., sh. I7. VI. 99, 2,300 ft. Diptera. Anthomyiidae: (2) Drymia hamata Fln., sh. I 7. VI. 99, 2,300 ft. (3) Trichophthicus sp., sh. and fp. 20. VI. 96, 23-2,400 ft. Coleoptera. (4) Meligethes aeneus F., sh. 17. VI. 99, 2,300 ft. Thysanoptera. (5) Thrips sp., 1 7. VI. $99,2,300 \mathrm{ft}$.

220. Stellaria media, Cyr. [Lit. Brit. 23,29 ; N.C.E. 1,3 b, 14, 18, 21 a, 21 b, 25, 33, Warnstorf 2507; Alps 2; N. Am. 12 b.] Always selffertilized in the absence of insect visitors.

Visitors. Hymenoptera. Petiolata parasitica. Ichncumonidae: (I) I sp., I 7.

${ }^{1}$ In August, 1905, on the Yorkshire moors north of Scarborough, about 40 per cent. of the flowers of Cerastium triviale then open had infertile stamens. 
IX. $95,800 \mathrm{ft}$. Braconidae: (2) I sp., sh. 24 . IX. 95, r,000 ft. (3) a second sp., Io. VI. 99, $700 \mathrm{ft}$. Diptera. Syrphidae: (4) Platychirus manicatus Mg., sh. I o. VI. 99, $700 \mathrm{ft}$. Muscidae: (5) Calliphora erythrocephala Mg., sh. I7. IX. 95, $800 \mathrm{ft}$. (6) Pollenia rudis F., sh. 22. IX. 95, $800 \mathrm{ft}$. Anthomyiidae: (7) Anthomyia radicum L., sh. Io. VI. 99, $700 \mathrm{ft}$. (8) Anthomyia sp., I7-2I. IX. 95, $800 \mathrm{ft}$. Coleoptera. (9) Meligethes viridescens F., sh. and fp. I6-22. IX. 95, $800 \mathrm{ft}$. Thysanoptera. (10) Thrips sp., sh. I 7-2 2. IX. 95, $800 \mathrm{ft}$.

221. Stellaria Holostea, Linn. [Lit. Brit. 23, $29 ;$ N.C.E. 1, 3 b, 18, 21 a, 21 b, 33, 34, 40 ; Alps 2 ; Pyren. 17.]

Visitor. Diptera. Anthomyiidae: (I) sp. fp. 10. VI. 99, $600 \mathrm{ft}$.

222. Stellaria graminea, Linn. [Lit. Brit. 23 ; N.C.E. 1, 3 b, 14, 18, 21 a, 21 b, 25, 33, 34; Alps 2.]

Visitors. Diptera. Syrphidae: (1) Platychirus manicatus Mg., 5. VII. 95, $800 \mathrm{ft}$. Anthomyiidae: (2) Trichophthicus sp., fp. I6. IX. 95, $900 \mathrm{ft}$.

223. Sagina procumbens, Linn. [Lit. Brit. 23 ; N.C.E. 14, 18, 21 a, 21 b, 34; Arct. 37 a.] Self-pollinating.

Visitor. Diptera. Chloropidae: (I) Oscinis ?, 2 2. VI. 95, $800 \mathrm{ft}$.

224. Spergula arvensis, Linn. [Lit. Brit. $23 ;$ N.C.E. 1, 3 b, 9, 14, 14 a, 18, 21 a, 21 b, 24, 33.] Schulz (in Ber. d. deutschen Bot. Gesellsch. $\mathrm{xxi}, 1903,119)$ points out how this plant is self-fertilized.

Visitors. Diptera. Syrphidae: (I) Platychirus manicatus Mg., 5. VII. 95 . (2) P. albimanus F., sh. 22. IX. 95. (3) Syritta pipiens L., 30. VI. 95. Tachinidae: (4) Siphona geniculata Deg., sh. 22. IX. 95. Anthomyiidae: (5) Trichophthicus sp., sh. 30. VI. 95. (6) Anthomyia sp., sh. 22. IX. 95. Thysanoptera. (7) Thrips sp., 30 VI. $95 ; 22$. IX. 95 . All at $800 \mathrm{ft}$.

\section{Class AB, § 60. Loiseleuria Type.}

225. Loiseleuria procumbens, Desv. [Lit. Arct. 34; 36, 37 a, 38 ; Alps 2,9.] The flower is slightly protogynous with ultimate self-fertilization on the fall of the corolla and sometimes before. The flowers are very little visited; Bombi pass them over in search of those of Vaccinium; they fill with water in bad weather. Yet each one sets seed. Like several other alpines which grow at Clova they are extremely variable, frequently being 6- or 7-merous.

Visitors. Diptera. Mycetophilidae: ( I) Sciara sp., four individuals sh. Bibionidae: (2) Scatopse sp. Anthomyiidae: (3) I sp. All I I. VI. 99, 2,400 ft.

Class AB (? Class Ne), $\S 6$ i. Salix Type.

226. Salix Caprea, Linn. [Lit. Brit. 23, 29 ; N.C.E. 1, 3 b, 16, 33, $34,40$.

Visitors to male catkins. Hymenoptera. Aculeata : Apidae: (I) Bombus lap- 
ponicus F., sh. 9-14. V. 98, 8-r,900 ft. (2) B. terrestris L., 9-II. V. 98, $800 \mathrm{ft}$. Diptera. Syrphidae: (3) Platychirus discimanus Lw., fp. and sh. 21. V. 97 ; 14. V. 98, 9-I,300 ft. (4) Eristalis arbustorum L., sh. I4. V. 98, $900 \mathrm{ft}$. Empidae: (5) Empis borealis L., sh. 2 r. V. $97,1,300$ ft. (6) E. niveipennis sh. 2 r. V. 97, I,300 ft. Muscidae: (7) Lucilia cornicina F., 21. V. 97, r,300 ft. Anthomyiidae: (8) Anthomyia sulciventris Ztt., sh. and fp. 2 I. V. 97, r,300 ft. (9) Anthomyia sp., 9-1 4. V. 98, 9-I,500 ft. Cordyluridae: (10) Scatophaga stercoraria L., sh. 9. V.98, 800 ft. Sepsidae: (I I) Sepsis cynipsea L., ? sh. 21. V. 97, r,300 ft. Hemiptera. (12) Anthocoris sp., sh. r 4. V. 98, $900 \mathrm{ft}$.

Visitors to female catkins. Hymenoptera. Aculeata: Apidae: (1) Bombus lapponicus F., sh. 9-16. V. 98, 8-r,400 ft. Diptera. Empidae: (2) Empis lucida Ztt., I6. V. 98, 800 ft. Chironomidae: (3) I sp., 16. V. 98, 800 ft. Anthomyiidae: (4) Anthomyia sp., 14. V. 98 . 1,400 ft. all sh.

227. Salix aurita, Linn. [Lit. Brit. 23, 29; N.C.E. 1,3 b, 16, 33.] This is well visited, especially its male catkins.

Visitors to male calkins. Hymenoptera. Aculeata: Apidae:(r) Apis mellifica L., cp. and sh. freq., I 9-2 7. V. $97 ; 7-16$. V. 98,6-900 ft. (2) Bombus hortorum L., 22. V. $97,700 \mathrm{ft}$. (3) B. lapponicus F., sh. 20-22. V. 97 ; I6. V. 98, 6-900 ft. (4) B. terrestris L., sh. 20-22. V. 97, 6-800 ft. Vespidae: (5) Vespa sp., 20. V. 97, $900 \mathrm{ft}$. Formicidae: (6) Formica fusca Latr., sh. 20. V. 97, $800 \mathrm{ft}$. Diptera. Syrphidae: (7) Melanostoma quadrimaculatum Verrall, 7.V.98,600 ft. (8) Chilosia bergenstammi Becker, sh. 22. V. 97, $600 \mathrm{ft}$. (8) Platychirus discimanus Lw., sh. and fp. 19-20. V. $97 ; 7-16$. V. 98, 8-900 ft. (9) Sericomyia lappona L., sh. 20. V. $97, \mathbf{1}, 200 \mathrm{ft}$. (10) Eristalis arbustorum L., sh. I6. V. 98, $700 \mathrm{ft}$. Empidae : (I I) Empis borealis L., 20-27. V. 97,6-I,200 ft. (I2) Rhamphomyia cinerascens Mg., sh. 20. V. 97, 9-1,200 ft. (r3) R. sulcata Fln., sh. 21. V. 96, 700 ft. Mycelophilidae: (14) Sciara sp., I6. V. 98, $600 \mathrm{ft}$. Bibionidae: (15) Scatopse sp., sh. 19. V. 97, $800 \mathrm{ft}$. Chironomidae: (16) I sp., 27.V.97, $700 \mathrm{ft}$. Muscidae: (I 7) Lucilia cornicina F., sh. 19-20. V. 97, 8-1,200 ft. (18) Pollenia vespillo F, sh. 20-27. V. 97 ; 16. V. 98, 7-r,200 ft. Anthomyiidae: (19) Anthomyia sulciventris Ztt., fp. and sh. 20-27. V. $97 ; 7$. V. 98, 7-900 ft. (20) Anthomyia sp., sh. 19. V. 97, $800 \mathrm{ft}$. (2I) Hylemyia ?, sh. 22. V. 97, $700 \mathrm{ft}$. Cordyluridae: (22) Scatophaga stercoraria L., 19.V. $97 ;$ 16. V. $98,7-800 \mathrm{ft}$.

Visitors to female catkins. Hymenoptera. Aculeata: Apidae: (I) Bombus terrestris L., sh. 22. V. $97 ; 7 . \mathrm{V}, 98,600 \mathrm{ft}$. (2) B. lapponicus F., 22. V. 97 ; 7-16. V. 98, 6-800 ft. (3) Andrena clarkella Kirby, sh. 22, V. 97, $600 \mathrm{ft}$. Diptera. Syrphidae: (4) Platychirus discimanus Lw. 19. V. 97; 7. V. 98, 6-1,300 ft. (5) Chilosia sparsa Lw., sh. 22. V. 97, 600 ft. Mycetophilidae: (6) Sciara sp., 16. V. 98, $600 \mathrm{ft}$. Muscidae: (7) Lucilia cornicina F., 20. V. 97, $900 \mathrm{ft}$. (8) Pollenia vespillo F., sh. 20. V. $97 ; 7 . \mathrm{V} .98,6-1,200 \mathrm{ft}$. Anthomyiidae: (9) I sp., 19-20. V. 97, 8-r,800 ft. Cordyluridae: (ro) Scatophaga stercoraria L., sh. 20-22. V. 97 ; 7-16. V. 98, 6-I,200 ft. Coleoptera. (I I) Phyllodecta vulgatissima L., sh. 22. V. $97,700 \mathrm{ft}$. 
228. Salix Lapponum, Linn. [Lit. Arct. 34.]

Visitors to male catkins. Hymenoptera. Aculeata: Apidae: (I) Bombus lapponicus F., sh. 24. V. 97, 2,800 ft. Vespidae: (2) Vespa norvegica F., I I. VI. 99, 2,100 ft. Diptera. Mycelophilidae: (3) Sciara sp., I I. VI. 99, 2, 100 ft. Chironomidae: (4) I sp., 24. V. $97,2,300 \mathrm{ft}$. Anthomyiïdae: (5) Coenosia sp., fp. 24 . V. 97 ; II. VI. 99,2 I-2,300 ft.

Visitor to female catkins. Diptera. Bibionidae: (I) Scatopse sp., I I. VI. 99, $2,100 \mathrm{ft}$.

229. Salix repens. Linn. [Lit. N.C.E. 1, 14, 25, 33, 34.]

Visitors to male catkins. Hymenoptera. Acüleata: Apidae: (I) Apis mellifica L., sh. 7 . V. $98,600 \mathrm{ft}$. (2) Bombus lapponicus F., sh. 22. V. 97, $700 \mathrm{ft}$. (3) B. terrestris L., 7. V. $98,600 \mathrm{ft}$. Sessiliventres. Tenthredinidae: (4) Nematus fallax Lep., sh. 18. V. 97, $800 \mathrm{ft}$. Diptera. Syrphidae. (5) Platychirus discimanus Lw., sh. and fp. freq. $2 \mathrm{I}-27 . \mathrm{V} .97 ; 7 . \mathrm{V} .98,6-\mathrm{I}, 400 \mathrm{ft}$. (6) Syrphus punctulatus Verrall, sh. 22. V. $97,700 \mathrm{ft}$. Empidae: (7) Empis borealis L., 20-22. V. $97,7-\mathrm{I}, 400 \mathrm{ft}$. (8) E. lucida Ztt., sh. 7. V. $98,600 \mathrm{ft}$. (9 and ro) Empis spp., sh. and fp., 21. V. 97 , I,400 ft. Muscidae: (I I) Lucilia cornicina F., sh. I8. V. $97,800 \mathrm{ft}$. (I 2) Pollenia vespillo F., sh. 7. V. $98,6-700 \mathrm{ft}$. Anthomyiidae: (г 3 ) Anthomyia sulciventris $\mathrm{Ztt}$., sh. and fp. 18-22. V. 97 ; 16. V. 98, 6-900 ft. (14 and I5) Anthomyia spp., sh. 20-27. V. $97 ;$ 1 4-16. V. $98,7-800 \mathrm{ft}$.

Visitors to female catkins. Hymenoptera. Aculeata: Apidae: (I) Bombus lapponicus F., sh. I 8-20. V. $97,800 \mathrm{ft}$. (2) B. terrestris L., sh. 22. V. 97 , 7. V. 98 , $700 \mathrm{ft}$. Diptera. Empidae: (3) Rhamphomyia cinerascens Mg., sh. 20. V. 97, 8-900 ft. Muscidae: (4) Lucilia cornicina F., 7. V. 98, 7co ft. (5) Pollenia vespillo F., 20. V. 97, $900 \mathrm{ft}$. Anthomyiidae: (6) Anthomyia sulciventris Ztt., sh. 20. V. 97 , $800 \mathrm{ft}$. (7 and 8) Anthomyia spp., 20-22. V. 97, 7-900 ft.

230. Salix phylicifolia, Linn. (including S. nigricans, Sm.) [Lit Arct. 7, 34, 36.]

Visilor to male catkins. Hymenoptera. Aculeata: Apidae: (I) Bombus lapponicus F., sh. I 3 . V. $98,700 \mathrm{ft}$.

Visilors to female catkins. Hymenoptera. Aculeata: Apidae: (I) Bombus lapponicus F., sh. I3. V. $98,800 \mathrm{ft}$. Diptera. Syrphidae: (2) Platychirus discimanus Lw., 2 I. V. 97, I, $300 \mathrm{ft}$. Empidae: (3) Rhamphomyia segmentata Ztt., sh. I3. VI. 99 , I, $400 \mathrm{ft}$. Chironomidae: (4) I sp., I3. V. 98, $700 \mathrm{ft}$. Anthomyiiddae: (5) Hyetodesia semicinerea W., sh. I 3. V. 98, 7-800 ft. (6) Anthomyia sulciventris Ztt., 2 r. V. 97 , 8-1,300 ft. (7 and 8) Anthomyia spp., I 3. V. 98, $700 \mathrm{ft}$. Cordyluridae: (9) Scatophaga stercoraria L., 2 I. V. 97, I,300 ft. Coleoptera. (I0) Telephorus obscurus L.; sh. I3. VI. 99, I, $400 \mathrm{ft}$.

231. Salix herbacea, Linn. [Lit. Arct. 36, 38; Alps 2, 9.] Fruits very freely.

Visitors to male catkins. Hymenoptera. Petiolata parasitica. Ichneumonidae: (I) I sp. Sessiliventres. Tenthredinidae: (2) Nematus ? capreae L., sh. Diptera. Chironomidae: (3 and 4) Ceratopogon spp., sh. Anthomyiidae: (5) Anthomyia sulciventris Ztt., sh. (6) Anthomyia sp. All 24. V. 97, 2,600 ft. 
Visitors to female catkins. Hymenoptera. Petiolata parasitica. Ichneumonidae: (I) A second individual of the same species that was caught on the male catkins, sh. Diptera. Anthomyiidae: (2) Anthomyia sp., as named above. Both 24. V. 97, $2,600 \mathrm{ft}$.

TABLE XL.

Actual number of Individuals visiting the different species in Class AB.

\begin{tabular}{|c|c|c|c|c|c|c|c|c|c|c|c|c|c|c|c|}
\hline & $\nabla$ & $\begin{array}{l}\widetilde{\Xi} \\
\infty\end{array}$ & $\cong$ & ลี & 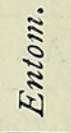 & $\stackrel{\Sigma}{\Sigma}$ & ड़े & $\begin{array}{l}\tilde{\nu} \\
\text { ปे }\end{array}$ & 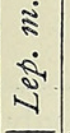 & $\begin{array}{l}5 \\
\text { वें }\end{array}$ & $\cong$ & $\tilde{\theta}$ & ن & 匈 & \\
\hline 4. I & - & - & - & - & 1 & - & - & - & - & - & - & 2 & 3 & - & \\
\hline 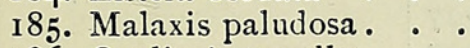 & 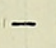 & - & - & - & 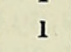 & - & - & - & - & - & - & - & - & & \\
\hline c & - & - & - & - & - & - & - & - & I & - & 3 & 9 & - & 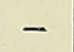 & \\
\hline $\mathrm{m}$ & - & - & - & - & - & - & - & - & - & - & - & 2 & - & - & \\
\hline aria & 5 & 4 & - & - & - & - & I & - & - & - & I & 6 & - & $\mathrm{I}$ & \\
\hline 1 & - & - & - & - & - & - & - & - & - & - & - & 7 & - & & \\
\hline . I & - & 2 & -1 & - & - & - & - & -1 & - & - & - & 8 & - & - & \\
\hline ats & I5 & I3 & - & - & I & - & 5 & 2 & - & - & & $4^{I}$ & $3^{\mathrm{I}}$ & - & \\
\hline . & - & - & - & - & 3 & - & - & - & - & - & 16 & 799 & I I I & 6 & \\
\hline $\cos$ & - & - & - & - & I & - & - & - & - & - & - & I & - & - & \\
\hline 1 & - & - & - & - & - & -1 & - & - & - & - & - & - & 5 & - & \\
\hline s & - & - & . & - & - & - & - & I & - & - & - & 10 & 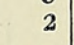 & $\mathbf{I}$ & \\
\hline $\mathrm{H}$ & - & - & - & - & - & - & - & 2 & - & - & I & - & 5 & - & \\
\hline tor is . & - & - & - & - & - & - & - & - & - & - & - & 2 & - & - & \\
\hline 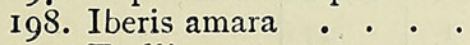 & - & - & - & - & - & - & - & 2 & - & - & 33 & 29 & 3 & - & \\
\hline 99. ' & - & - & - & - & I & - & - & - & - & - & I & 2 & 8 & - & \\
\hline narius & - & - & - & -1 & - & - & - & I & - & - & - & - & - & - & \\
\hline$S$ & - & - & - & - & 3 & - & - & - & - & - & 2 & IC & 8 & - & \\
\hline I & - & - & - & - & 2 & - & - & - & - & - & 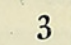 & I & 6 & - & \\
\hline 1 & - & - & - & - & - & - & - & - & - & - & 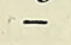 & I4 & 22 & - & \\
\hline mus & & - & - & - & - & - & - & - & -. & - & - & & - & - & \\
\hline la & 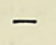 & - & - & - & - & - & - & I & - & 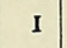 & 6 & 6 & 15 & - & \\
\hline is & - & - & - & - & - & - & - & - & - & - & - & 1 & & - & \\
\hline & - & I & I & 26 & 9 & - & - & 2 & - & I & $4^{8}$ & 1414 & I $3 \mathrm{I}$ & 6 & \\
\hline 1 & - & - & - & 3 & - & - & - & - & - & - & 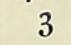 & & 19 & - & \\
\hline $\mathrm{R}$ & - & - & - & - & - & - & - & - & - & - & - & 35 & - & & \\
\hline $\mathrm{E}$ & - & $\cdots$ & - & - & - & - & - & - & - & - & - & & - & - & \\
\hline rum & - & - & - & - & 2 & - & - & - & - & - & - & & - & - & \\
\hline lla & - & I & 6 & - & 14 & $\mathrm{I} 7$ & - & 4 & I & 5 & I9 & 286 & 8 & I & \\
\hline$E$ & - & - & - & - & - & ${ }^{\circ}$ & - & -1 & - & - & 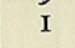 & & - & & \\
\hline I & - & - & I & - & I & - & - & - & - & - & & & 6 & & \\
\hline & & - & - & - & 3 & & - & - & - & - & 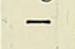 & . & t & IO & \\
\hline 5 & - & - & - & - & I & - & - & - & - & - & - & - & 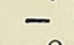 & - & \\
\hline & - & - & - & I & IO & $1-$ & - & I & $\mathrm{I}$ & - & I 2 & 7 & 48 & & \\
\hline & - & - & - & - & 4 & - & - & - & - & - & & 11 & . & & \\
\hline $\mathrm{n}$ & - & - & - & - & I & - & - & - & - & - & - & & & & \\
\hline & - & - & - & - & 6 & - & - & - & - & - & & & & 5 & \\
\hline & - & - & - & - & - & - & - & - & - & - & - & 1 & 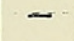 & & \\
\hline & - & - & - & - & - & - & - & - & - & - & & I & - & - & \\
\hline & - & - & - & - & - & - & - & - & - & - & 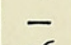 & 1 & - & - & \\
\hline & - & - & $\pi$ & - & - & - & - & - & - & - & It & & - & 2 & \\
\hline is procumbens & - & - & & - & - & - & - & -1 & - & - & - & & & & \\
\hline
\end{tabular}

Class AB. Section Ne.

226. Salix Caprea . . . 228. Salix aurita . . . 4767 I 6 - 1 I 229. Salix repens ... 230. Salix phyllicifolia 23I. Salix herbacea.

Total .... 68


TABLE XLI.

To show how class $A B$ obtains visits from less than its share in proportion of decidedly desirable insects and more of indifferent insects.

\begin{tabular}{|c|c|c|c|c|}
\hline & \multicolumn{2}{|c|}{ Available. } & \multicolumn{2}{|c|}{ To Class $A B$. } \\
\hline & No. & $\%$ & No. & $\%$ \\
\hline Decidedly desirable & 1,763 & IC.19 & 205 & $4 \cdot 84$ \\
\hline Desirable. . & 1,277 & $7 \cdot 37$ & 276 & $6 \cdot 5^{2}$ \\
\hline Indifferent . . & 12,993 & 75.08 & $3,5^{68}$ & $84 \cdot 27$ \\
\hline Injurious . & 1,273 & $7 \cdot 36$ & 185 & $4 \cdot 37$ \\
\hline
\end{tabular}

TABLE XLII.

Insects visiting class $\mathrm{AB}$ in different seasons classed by desirability.

\begin{tabular}{|c|c|c|c|c|c|c|}
\hline & \multicolumn{2}{|c|}{ Spring. } & \multicolumn{2}{|c|}{ Summer. } & \multicolumn{2}{|c|}{ Auiumn. } \\
\hline Decidedly desirable & I 88 & $16 \cdot 33$ & 16 & .59 & I & .27 \\
\hline Desirable. . & 99 & 8.60 & 159 & 5.85 & 18 & 4.89 \\
\hline Indifferent & 848 & $73 \cdot 73$ & $2,42 \mathrm{I}$ & $89 \cdot 17$ & 299 & $8 \mathrm{I} \cdot 02$ \\
\hline Injurious . & I5 & $I \cdot 30$ & iI9 & $4 \cdot 3^{8}$ & $5^{I}$ & I 3.82 \\
\hline Total . . & 1,150 & & $2,7 \times 5$ & & $3^{69}$ & \\
\hline
\end{tabular}

\section{TABLE XLIII.}

Percentage of different groups of insects visiting Class $\mathrm{AB}$ in different seasons.

\begin{tabular}{|c|c|c|c|c|c|c|c|c|c|c|c|c|}
\hline & $\stackrel{\widetilde{Z}}{\widetilde{\nabla}}$ & 咅 & $\stackrel{\Sigma}{\Sigma}$ & 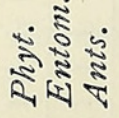 & हैं & نें & $\begin{array}{l}\Sigma \\
\Sigma \\
\text { ปे }\end{array}$ & $\begin{array}{l}\dot{\vdots} \\
\dot{\mathrm{v}}\end{array}$ & ฐ & 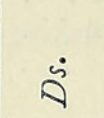 & $\ddot{0}$ & มี \\
\hline Spring & $5 \cdot 9 \mathrm{I}$ & 10.43 & .07 & .86 & .17 & - & - & - & $8 \cdot 5^{2}$ & 72.26 & $\mathrm{I} \cdot 3^{\circ}$ & \\
\hline Summer & - & - & .29 & $3 \cdot 64$ & .22 & $\cdot 5^{8}$ & . II & $\cdot 25$ & $5 \cdot 45$ & 79.11 & $9 \cdot 57$ & .73 \\
\hline Autumn & - & .27 & - & $2 \cdot 9^{8}$ & - & - & - & - & 4.87 & $62 \cdot 33$ & 18.69 & IC. 84 \\
\hline
\end{tabular}

\section{TABLE XLIV.}

Desirability of visitors to Class $\mathrm{AB}$ according to colour, in percentages.

\begin{tabular}{|c|c|c|c|c|}
\hline & $\begin{array}{c}\text { Rose and } \\
\text { purple }\end{array}$ & Yellow. & White. & Green. \\
\hline Decidedly desirable & 一 & $\cdot 4 \mathrm{I}$ & $1 \cdot 10$ & 30.96 \\
\hline Desirable. . . & - & $3 \cdot 3^{I}$ & 16.08 & 16.23 \\
\hline Indifferent . & $87 \cdot 50$ & $93 \cdot 4^{\circ}$ & $64 \cdot 9^{8}$ & 50.83 \\
\hline Injurious . & 12.50 & 2.87 & $17 \cdot 84$ & 1.98 \\
\hline
\end{tabular}


TABLE XLV.

Percentage of different groups of insects visiting flowers of different colours in Class AB.

\begin{tabular}{|c|c|c|c|c|c|c|c|c|c|c|c|c|}
\hline & $\frac{\tilde{R}_{2}^{2}}{\mathbb{R}^{2}}$ & $\underset{\Xi}{\stackrel{\Xi}{0}}$ & $\lesssim$ & 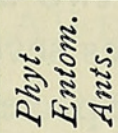 & $\vec{s}$ & ปें & $\begin{array}{l}\text { है } \\
\text { ปั }\end{array}$ & $\dot{\vdots}$ & $\approx$ & $\tilde{a}$ & $\tilde{0}$ & 诘 \\
\hline Rose-purpl & - & - & - & I 2.33 & - & - & - & - & - & $87.5^{6}$ & - & - \\
\hline $\mathrm{Ye}$ & 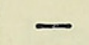 & . 12 & .22 & 2.39 & - & .28 & .03 & .22 & 3.06 & 86.58 & 6.59 & $\cdot 47$ \\
\hline White . & - & - & - & $7 \cdot 4^{8}$ & - & I. 10 & .44 & - & $I_{5} \cdot 63$ & $44 \cdot 7 \mathrm{I}$ & 20.26 & 10.35 \\
\hline Green . & II $\cdot 25$ & 19.37 & $\cdot 33$ & $I \cdot 49$ & $I \cdot 3^{2}$ & $\cdot 33$ & - & - & I 5.89 & $42 \cdot 54$ & 6.95 & $\cdot 49$ \\
\hline
\end{tabular}

In Class AB, Tables XLIV and XLV show that green flowers get the best visitors, and white flowers get, on the whole, better visitors than yellow flowers. In Table XLII it is seen that in spring many high types of insects go to $\mathrm{AB}$. This is due to the frequent visits of $\mathrm{Apis}$ and Bombus to Acer Pseudo-platamus and Salices-green flowers. If we take away Salix from Class AB, then we obtain Table XLVI :

\section{TABLE XLVI.}

Insects visiting Class AB (Salix excluded) in different seasons classed by desirability.

\begin{tabular}{|c|c|c|c|c|c|c|}
\hline & \multicolumn{2}{|c|}{ Spring. } & \multicolumn{2}{|c|}{ Summer. } & \multicolumn{2}{|c|}{ Autumn. } \\
\hline Decidedly desirable & $4^{\circ}$ & $5 \cdot 46$ & I6 & .59 & I & .27 \\
\hline Desirable. . & 7 & .95 & 158 & 5.83 & 18 & 4.89 \\
\hline Indifferent & $67 \mathrm{I}$ & $9^{I} \cdot 54$ & $2,4^{18}$ & $89 \cdot 19$ & 299 & $8 \mathrm{I} \cdot 02$ \\
\hline Injurious & I 5 & 2.05 & iI 9 & $4 \cdot 39$ & $5 \mathrm{I}$ & I 3.82 \\
\hline Total & 733 & & $2,7 \mathrm{II}$ & & $3^{69}$ & \\
\hline
\end{tabular}

And if we also take away Acer Pseudo-platamus we leave hardly any insects of high type for spring.

The visitors to Salix which are, all but four, spring insects are :-

\begin{tabular}{|c|c|c|}
\hline Decidedly desirable & 148 & $34 \cdot 34 \%$ \\
\hline Desirable & 93 & $21.58 \%$ \\
\hline Indifferent . & I $8 \mathrm{I}$ & $42.00 \%$ \\
\hline Injurious . & 9 & $2.08 \%$ \\
\hline
\end{tabular}

Class $A B$ attracted four species of the butterflies, four of the larger moths, and four of the smaller, including Eriocephala; of Hymenoptera, A pis, three species of Bombus, three of Andrena, two of Vespas, two ants, some eight or more species of parasitic Hymenoptera and four Tenthredinidae : among Diptera, in the Syrphidae, of one species of Sericomyia, two of Eristalis, three of Chilosia, four of Syrphus, a Chrysogaster, five of Platychirus, two of Melanostoma, two of Sphaerophoria, Syritta, and one of Ascia ; in the Empidae of ten species of Empis, four of Rhamphomyia, two of Hilara and one of Clinoceira ; in 'Tachinidae two species of Siphona ; in 
Muscidae, two species of Pollenia, one of Lucilia and one of Calliphora ; in Sarcophagidae a Sarcophaga ; in Cordyluridae three species of Scatophaga ; in Sciomyzidae, one of Tetanocera, in Anthomyiidae twenty-three species including Drymia hamata; in Tipulidae two species; in Bibionidae two, in Dolichopodidae three; and of small flies seventeen; among Coleoptera eighteen species; among Hemiptera four species; among Neuroptera two species; among Thysanoptera Thrips; and among Araneida a crab-spider and a harvester spider.

\section{Class Po. AB, $\S 62$. Erect AB-like type, with much Pollen.}

232. Helianthemum vulgare, Linn. [Lit. Brit. 23, $39 ;$ N.C.E. 1,3 b, 11, 33, Warnstorf 2507; Alps 2, 16,34; Pyren. 17.]

Visitors. Lepidoptera. Heterocera: Tortricidae: (r) Tortrix sp. seeking h. 2. VI. $95,800 \mathrm{ft}$. Eriocephalidae: (2) Eriocephala calthella L., fp. 2 I. VII. $95,900 \mathrm{ft}$. Hymenoptera. Aculeata: Apidae: (3) Apis mellifica L., twice fp. 25. VI. $96,700 \mathrm{ft}$. (4) Andrena analis Panz., 20. VI. 95, I,000 ft. Diptera. Syrphidae: (5) Platychirus manicatus Mg., seeking h. 3. VII. 95 ; 1 9. VI. 99, 8-I,000 ft. (6) Chrysogaster hirtella Lw., fp. 18. VI. 96, $800 \mathrm{ft}$. (7) Sphaerophoria picta Mg., 3. VII. 95, 1,200 ft. (8) Ascia sp. fp. 25. VI. 96, $700 \mathrm{ft}$. Mycetophilidae: (9) Sciara sp. 4. VII. 94, about $800 \mathrm{ft}$. Anthomyiidae: (10) Hyetodesia lucorum Fln., fp. 29. VI. 95, $800 \mathrm{ft}$. (I I) Hyetodesia sp., 4. VII. 94; 3. VII. 95, about 8-1,000 ft. (I 2) Limnophora sp., seeking h. and fp. 22. VI. $95,800 \mathrm{ft}$. (13) Drymia hamata Fln., fp. 20. VI. 95, I,000 ft. (14) Trichophthicus hirsutulus Ztt., fp. 24 . VI.-23. VII. 95 ; 8. VII. 96, 8-I,200 ft. (I 5) Hylemyia nigrescens Rnd., I 5-I6. VI. 99, $800 \mathrm{ft.}$ (I6) Anthomyia radicum L., 4. VII. 94, about $800 \mathrm{ft}$. (I 7 and $\mathrm{I} 8$ ) Anthomyia spp. fp. 4. VII. 94 ; I 4 . VI.-3. VII. 95 ; 16. VI.-1 1. VII. 96 ; 19. VI. $99,7-1,300$ ft. (1 9 ) Homalomyia incisurata Ztt., 4. VII. 94, about $800 \mathrm{ft}$. (20) Coenosia sp., 4. VII. 94, about $800 \mathrm{ft}$. Coleoptera. (2 I) Meligethes viridescens F., 4. VII. 94, about $800 \mathrm{ft}$. Neuroptera. (22) Chloroperla sp., 4. VII. 94, about $800 \mathrm{ft}$.

\section{Hypericum pulchrum, Linn. [Lit. Brit. 23; N.C.E. 14, 18.]}

Visitors. Hymenoptera. Aculeata: Apidae: (I) Andrena analis Panz., 22. VI. 96, I,000 ft. Diptera. Syrphidae: (2) Sphaerophoria sp., 28. V. 95, I,000 ft. Anthomyiidae: (3) Hyetodesia incana W., fp. 27 . VI. 95 ; 29. VI. 96, I I-I,800 ft. (4) Trichophthicus sp., fp. $\hat{\jmath}-1$ 7. VII. 95 ; 29. VI. $96,8-I, 500 \mathrm{ft}$. (5) Anthomyia sp., fp. 24. IX. 95, $900 \mathrm{ft}$.

234. Rosa mollis, Sm. ( $R$. villosa, Linn.). [Lit. of $R$. canina, sensu ampliore, Brit. 23 ; N.C.E. 1, 3 b, 8, 14, 18, 34.]

Visitors. Hymenoptera. Aculeata: Apidae: (I) Bombus lapponicus F., seeking h. I0. VII. 96, 2, 100 ft. (2) B. terrestris L., cp. and seeking h. 26 . VI. $95 ; 26$. VI. 96, 8-900 ft. Diptera. Syrphidae: (3) Eristalis rupium F., seeking h. 25-26. VI. 95, $800 \mathrm{ft}$. (4) E. arbustorum L., 8. VII. 95, $800 \mathrm{ft}$. Anthomyiidae: (5) Hyetodesia incana W., 26. VI. $96,900 \mathrm{ft}$. (6) H. semicinerea W., 3. VII. $95,800 \mathrm{ft}$. (7) Drymia hamata Fln., seeking h. 24. VI. $95,800 \mathrm{ft}$. (8) Trichophthicus sp., fp. and 
seeking h. 2 I. VI.-4. VII. 95, $800 \mathrm{ft}$. (9) Anthomyia sp., fp. and seeking h. 4-5. VII. 95; 24-25. VI. 96, 7-800 ft. Thysanoptera. (Iо) Thrips sp., 2 I. VI.-I I. VII. 96, 7-800 ft. Neuroptera. (I I) Chloroperla sp., 2. VII. 95, $800 \mathrm{ft}$.

Class Po. AB, $\$ 63$. AB-Like Type, with massed Flowers.

235. Spiraea Ulmaria, Linn. [Lit. Brit. 23,39 ; N.C.E. 1,3 b, 8,11 , 14 a, 16, 18, 21 a, 21 b, 33, 34, 40 ; Arct. 36 ; Alps, 2 ; Pyren. 17.]

Visitors. Lepidoptera. Heterocera: Eriocephalidae: (I) Eriocephala calthella L., fp. 6-I 7. VII. 95, 7-800 ft. Hymenoptera. Aculeata: Apidae: (2) Apis mellifica L., cp. and seeking h., I 5-22. VII. 95; 3-II. VII. 96, 7-800 ft. freq. (3) Bombus lapponicus F., seeking h. 4-I I. VII. 96, 800 ft. (4) B. terrestris L., cp. I 5. VII. 95 ; I I. VII. 96, 7-800 ft. Vespidae: (5) Vespa norvegica F., seeking h. 4-I I. VII. 96, $800 \mathrm{ft}$. Sessiliventres. Tenthredinidae: (6) Allantus arcuatus Forst., 29. V. 96, $900 \mathrm{ft}$. Diptera. Syrphidae: (7) Eristalis arbustorum L., fp. and seeking h., I 5-1 7. VII. 95; 25. VI. 96, 7-800 ft. Muscidae: (8) Calliphora vomitoria L., 3. VII. 96, $800 \mathrm{ft}$. Anthomyiidae: (9) Hyetodesia incana W., fp. and seeking h., I 5. VII. 95 ; 3-r r. VII. 96, $800 \mathrm{ft}$. (I0) Limnophora sp., 2. VII. 95, $800 \mathrm{ft}$. (I I) Trichoph hicus sp., fp. I 2. VII. 95 ; 3. VII. 96, 7-800 ft. (I 2 and $\mathbf{I}_{3}$ ) Anthomyia spp., fp. 4-I 5. VII. 95 ; I I. VII. 96, 7-800 ft. Cordyluridae: (14) Scatophaga maculipes Ztt, fp. I 5. VII. 95, $800 \mathrm{ft}$.

Class Po. AB, $\S 64$. AB-like Type with little Pollen.

236. Lysimachia punctata, Linn. An escape from cultivation.

Visitor. Diptera. Chironomidae: ( I) Ceratopogon flavipes Mg., seeking h., 2. VII. $95,800 \mathrm{ft}$.

237. Lysimachia nemorum, Linn. [Lit. Brit. 23 ; N.C.E. 18, 33.]

Visitors. Hymenoptera. Aculeata: Formicidae: (I) Formica fusca Latr., seeking h., 29. VI. 96, I,500 ft. Diptera. Syrphidae: (2) Syrphus sp., 23. VI. 96, $900 \mathrm{ft}$. Chironomidae: (3) Orthocladius sp., 29. VI. 95, I,100 ft. Anthomyiidae: (4) I sp., fp. Io. VI. 99, $700 \mathrm{ft}$.

238. Trientalis europaea, Linn. [Lit. N.C.E. 1, 3 c, 4, 21 a, 21 b; Arct. 36.] The stamens and style are of equal length and are ripe together, but are not near enough to bring about spontaneous selfpollination.

Visitors. Diptera. Syrphidae: (I) Platychirus albimanus F., seeking h., 27. VI. 95, 2,100 ft. (2) Syrphus vitripennis Mg., fp. I 5. VI. 99, I,200 ft. Empidae: (3) Empis tessellata F., seeking h. I 9. VI. 96, I,500 ft. Anthomyizidae: (4) Anthomyia sp., fp. 22. V. 96 ; 1 0 . VI. $99,7-1,000$ ft. Coleoptera. (5) Telephorus paludosus Fall., 20. VI. 95, I,500 ft. Thysanoptera. (6) Thrips sp., Io. VI. 99, $700 \mathrm{ft}$.

239. Narthecium Ossifragum, Huds. [Lit. Brit. 23, 39 ; N.C.E. 3 a, 14, 14 a, 18 ; Alps 9.]

Visitors. Lepidoptera. Heterocera: Eriocephalidae: (1) Eriocephala calthella 
L., fp. 28. VI.-6. VII. 95, $800 \mathrm{ft}$. Hymenoptera. Aculeata: Apidae : (2) Bombus hortorum L., seeking h., I I. VII. 96, once $800 \mathrm{ft}$. Diptera. Syrphidae: (3) Platychirus manicatus Mg., fp. 6. VII. 95, $900 \mathrm{ft}$. Anthomyiidae : (4) Hyetodesia incana W., fp. 6-I I. VII. 96, 8-900 ft. (5) Hyetodesia sp., 2. VII. 95, $900 \mathrm{ft.} \mathrm{(6)} \mathrm{Tri-}$ chophthicus sp., fp. 26 . VI.-6. VII. $95 ;$ I-6. VII. $96,9-1,500 \mathrm{ft}$.

TABLE XLVII.

Total number of visitors to AB-like flowers of Class Po.

\begin{tabular}{|c|c|c|c|c|c|c|c|c|c|c|c|c|c|c|c|}
\hline Class Po. $(A B)$ & 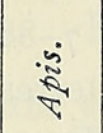 & $\stackrel{8}{0}$ & 亲 & $\mid$ & 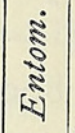 & $\frac{\pi}{3}$ & $\begin{array}{l}0 \\
0 \\
0 \\
0\end{array}$ & 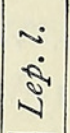 & $\left|\begin{array}{c}2 \\
5 \\
2 \\
2 \\
2\end{array}\right|$ & $\begin{array}{l}\dot{1} \\
\dot{8}\end{array}$ & ฐ & $\ddot{a}$ & 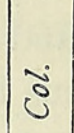 & 《ัँ & ชั \\
\hline $\begin{array}{l}\text { 38. Trientalis europaea . } \\
\text { 39. Narthecium Ossifragnm }\end{array}$ & $\begin{array}{l}\frac{2}{-} \\
\frac{4^{6}}{-} \\
\overline{-} \\
-\end{array}$ & $\begin{array}{c}- \\
- \\
5 \\
4 \\
- \\
- \\
- \\
1\end{array}$ & $\begin{array}{r}I \\
I \\
- \\
- \\
- \\
-\end{array}$ & $\begin{array}{l}- \\
- \\
- \\
I \\
\bar{z} \\
- \\
-\end{array}$ & $\begin{array}{l}\bar{z} \\
\overline{-} \\
\overline{-} \\
\overline{-} \\
\bar{z} \\
\overline{-}\end{array}$ & $\begin{array}{c}- \\
- \\
- \\
- \\
- \\
I \\
-\end{array}$ & $\begin{array}{l}\overline{-} \\
\bar{z} \\
\text { I } \\
\text { z } \\
\text { z }\end{array}$ & $\begin{array}{l}\bar{z} \\
\overline{-} \\
\overline{-} \\
\bar{z} \\
\bar{z} \\
\overline{-}\end{array}$ & $\begin{array}{c}I \\
- \\
- \\
- \\
- \\
-\end{array}$ & $\begin{array}{c}\text { I } \\
\overline{-} \\
- \\
- \\
- \\
\text { I1 }\end{array}$ & $\begin{array}{c}8 \\
\mathbf{I} \\
3 \\
3 \\
-1 \\
\mathbf{I} \\
3 \\
\mathbf{I}\end{array}$ & $\begin{array}{r}55 \\
15 \\
17 \\
24 \\
1 \\
2 \\
12 \\
16\end{array}$ & $\begin{array}{c}- \\
\bar{z} \\
\overline{-} \\
\overline{-} \\
\bar{x} \\
-\end{array}$ & $\begin{array}{c}- \\
- \\
- \\
- \\
- \\
-\end{array}$ & $\begin{array}{l}68 \\
\text { I } \\
28 \\
85\end{array}$ \\
\hline Total . . . . & 48 & 10 & 2 & I & - & I & $\mathbf{I}$ & - & I & 18 & 20 & $14^{2}$ & $\mathbf{I}$ & 4 & 249 \\
\hline Percentages & $19 \cdot 28$ & 4.02 & .80 & $\cdot 40$ & - & & .40 & - & & $7 \cdot 23$ & 8.03 & $57 \cdot 03$ & $\cdot 40$ & $\mathrm{I} \cdot 6 \mathrm{I}$ & \\
\hline
\end{tabular}

\section{Class A, $\S 65$. Open Saxifraga Type.}

240. Saxifraga aizoides, Linn. [Lit. N.C.E. 1 ; Arct. 7, 33, 37 b ; Alps 2, 16 ; Pyren. 17.] Proterandrous with ultimate self-pollination. The stamens are of the usual two lengths, and go through the usual process of coming up singly to dehisce at the centre of the flower and finally coming up together to effect self-pollination.

Visitors. Lepidoptera. Heterocera: Noctuidae: (r) Hadena gemina Hb., sh. 18. VI. 96, $800 \mathrm{ft}$. Tortricidae: (2) I sp., sh. 22. VI. 96, 23-2,400 ft. Eriocephalidae: (3) Eriocephala calthella L., 28. VI.-I I. VII. 96 ; 26. VI.-10. VII. 96, 800-2, 400 ft. Hymenoptera. Aculeata: Apidae: (4) Bombus lapponicus F., sh. I. VII. 95, $800 \mathrm{ft}$. and $\mathrm{I}-\mathrm{I}$. VII. 96 , I 9-2,200 ft. (5) B. terrestris L., I 4. IX. $95, \mathrm{I}, 800 \mathrm{ft}$. (6) Andrena fucata Sm., sh. 23. VII. 95, $800 \mathrm{ft}$. Vespidae: (7) Vespa norvegica F., sh. 6-23. VII. 95; 4.--ro. VII. 96, 800 ft. Formicidae: (8) Formica fusca Latr., sh. very ab. 2 r. VI.-23. VII. $95 ; 20-23$. IX. $95 ; 23$. VI.-IO. VII. 96, 8-2,400 ft. Myrmicidae: (9) Myrmica rubra L., sh. freq. 22. VI.-I I. VII. 95; 1 4-18. IX. 95; 5. VII. 96, 8-1,700 ft. Petiolata parasitica: Ichneumonidae: (ro) Ichneumon molitorius Grav., sh. I 8-24. IX. 95, 9-1,800 ft. (I I) Hemiteles micator Grav., sh. I 9. IX. 95, 2, 400 ft. (I 2) Pimpla examinator F., I 8. IX. 95, 800 ft. (I3) Amblyteles subsericans Grav., sh. 1. IX. $95,1,500$ ft. (1 4, 15, I6, 17, I 8, and 19) 6 spp. sh. 4 . VII. $94 ; 1-23$. VII. 95 ; 14-23. IX. 95 ; I8. VI.-10. VII. 96, 800-2,600 ft. Chalcididae: $(20$ and 21 ) $2 \mathrm{sp}$. sh. 22. VI.-10. VII. 96, 23-2,400 ft. Cynipidae: (22) I sp., 23. VII. 95, $800 \mathrm{ft}$. Sessiliventres: Tenthredinidae: (23) Tenthredo olivacea Klug., 6. VII. 95, 2,500 ft. 
Diptera. Syrphidae: (24) Chilosia fraterna Mg., sh. 22. VI. 95 ; 8. VI. 96 ; I 5. VI. 99, 8-r, ro० ft. (25) Syrphus ? ribesii L., sh. 8. VI.-ro. VII. 96, 8-2,300 ft. (26) S. ? vitripennis Mg., sh. 23. VII. 95, $800 \mathrm{ft}$. (27) Sphaerophoria scripta L., sh. 23. VII. 95, $800 \mathrm{ft}$. (28) Sericomyia borealis Fln., 2. VII. 96, $800 \mathrm{ft}$. (29) Eristalis arbustorum L., sh. 1 8. VI. 96, 800 ft. (30) Syritta pipiens L., sh. 18. VI.-10. VII. 96, 800 ft. Empidae: (3r) Empis tessellata F., sh. 26. VI. 95; 2. VII. 96, 8-900 ft. (32) E. punctata Mg., sh. 23. VII. 95, $800 \mathrm{ft}$. (33) Rhamphomyia albosegmentata Ztt., 26 . VI. 95, 1,000 ft. (34) R. ? sulcata Fln., 4. VII. 94, $800 \mathrm{ft}$. (35) Rhamphomyia sp., sh. 6. VII. 96, $800 \mathrm{ft}$. (36) Hilara matrona Hal., sh. 23. VII. 95, $800 \mathrm{ft}$. (37) Hilara sp., sh. 4. VII. 94; 4. VII. 95, 800 ft. Mycetophilidae: (38) Sciara sp., sh. 4-23. IX. 95 ; I. VII. 96, I I-2,200 ft. Bibionidae: (39) Scatopse sp., sh. 6. VII. 96, 2, I00 ft. (40) Dilophus albipennis Mg., 22. VI.-I. VII. 95; 26-27. VI. 96, 8-900 ft. (4 I) Bibio pomonae F., sh. 24. IX. 95 ; 1 0 . VII. $96,800 \mathrm{ft}$. Simuliidae: (42) Simulium sp., 25 . VI. 96, 2,200 ft. Chironomidae: (43) Corynoneura sp., sh. 4. VII. 94, I8. VI. 96, $800 \mathrm{ft}$. (44) Ceratopogon sp., 4. VII. 94, 800 ft. Psychodidae: (45) Psychoda sp., 22. VI. 95, I I-I,800 ft. (46) Psychoda ?, 2 I. IX. 95, I, I00 ft. Limnobidae: (47) Limnophila meigenii Verrall, sh. 23. IX. 95, I,400 ft. Dolichopodidae: (48) Dolichopus signatus Mg., sh. 28. VI. 95, 800 ft. (49) D. atratus Mg., sh. 4. VII. 94 ; 28. VI. 95, $800 \mathrm{ft}$. (50) D. pennatus Mg., sh. 28. VI. 95, $800 \mathrm{ft}$. (5 I) Dolichopus sp., sh. I 8. VI.-6. VII. 96, 8-2,200 ft. (52) Argyra argentina Mg., sh. 23. VII. 95, $800 \mathrm{ft}$. Tachinidae: (53) Echinomyia fera L., sh. I. VII. 95, $800 \mathrm{ft}$. Sarcophagidae: (54) Sarcophaga sp., 2. VII. 96, $800 \mathrm{ft}$. (55) Cynomyia mortuorum L., sh. I-10. VII. 95, 8-2,200 ft. (56) C. alpina Ztt., ro. VII. 95, 2,200 ft. Muscidae: (57) Lucilia cornicina F., sh. 24. IX. 95, I 7-1,800 ft. (58) Calliphora cognata Mg., sh. Io. VII. 95, 2,200 ft. (59) C. erythrocephala Mg., I 8. VI.-6. VII. $96,800 \mathrm{ft}$. (60) C. vomitoria L., sh. 4-23. VII. 95, $800 \mathrm{ft}$. (6r) Pollenia rudis F., sh. 24. IX. 95, 1,800 ft. Anthomyizdae : (62) Hyetodesia incana W., sh. freq. 4. VII. $94 ; 22$. VI.-23. VII. 95 ; 8. IX. 95 ; 18. VI.-4. VII. 96, 8-2,200 ft. (63) H. lucorum Fln., 4. VII. 94, $800 \mathrm{ft}$. (64) H. errans Mg., 4. VII. 94. (65) Limnophora solitaria Ztt., sh. 26. VI.-10. VII. 96, 18-2,300 ft. (66) Drymia hamata Fln., 25. VI. 95 ; I 4. IX. 95, 800 ft. (67) Trichophthicus sp., sh. 6. VII. 95 ; 18. IX. 95, 8-I,300 ft. (68) Anthomyia sulciventris Ztt., 22. VI.-23. VII. 95, 8-900 ft. (69) A. radicum L., 4. VII. 94, 800 ft. ( $70,7 \mathrm{I}$, and 72$)$ Anthomyia spp., sh. 4. VII. 94 ; 6-I 3. VII. 95 ; I 4-24. IX. 95 ; r 9. VI.-1 o. VII. 96, 8-2,500 ft. (73) Coenosia sp., sh. 4. VII. 95, 800 ft. - Cordyluridae: (74) Scatophaga stercoraria L., fp. sh. 4. VII. 94 ; 6. VII. 95 ; I 4-23. IX. $95 ; 2-10$. VII. $96,8-2,500$ ft. (75) S. squalida Mg., sh. 28. VI. $95,800 \mathrm{ft}$. Ortalidae: $(76)$ Pteropaectria frondescentiae L., 22. VI. $95 ;$ I 8-22. VI. 96, 8-2,300 ft. Sepsidae: (77) Sepsis cynipsea L., 14. IX. 95, 1,500 ft. Ephydridae: (78) Hydrellia griseola Fln., sh. 28. VI. 95, 1,700 ft. Chloropidae: (79) Chloropisca ornata Mg., I. VII. 95, 800 ft. Phoridae: (80) Phora rufipes Mg., sh. 22. VII. 95, 800 ft. (8I) Phora sp., sh. I 4-23. IX. 95, 8-I,800 ft. Coleoptera. (82) Meligethes viridescens F., sh. and fp. 28. VI. 95 ; I6-2 I. IX. 95; 26. VI.-6. VII. 96, 8-2,300 ft. (83) Sericosomus brunneus L., sh. 22. VI.-r. VII. 95, 9-r, roo ft. (84) Donacia discolor Panz., sh. 23. VII. 95, $800 \mathrm{ft}$. (85) D. sericea L., sh. 23. VII. 95, $800 \mathrm{ft}$. (86) Anthophagus alpinus Payk., sh. 6-r 3. VII. 95 ; 6. VII. 96, 20-2,400 ft. (87) Epuraea aestiva L., 23. IX. 95, 2,000 ft. (88) Telephorus pellucidus F., sh. 2. VII. 
95, $800 \mathrm{ft}$. (89) Corymbites cupreus F., 4. VII. 94, $800 \mathrm{ft}$. Araneida. (90) Oligolophus morio F., sh. 23 . IX. 95, I 4-2,000 ft.

241. Saxifraga umbrosa, Linn. [Lit. N.C.E. Plateau 2002 ; Alps 2.] An escape from cultivation.

Visitors. Hymenoptera. Petiolata parasitica : (I) I sp. Diptera. Syrphidae: (2) Syritta pipiens L., sh. Anthomyiidae: (3) I sp. All I 8. VI. 96, $900 \mathrm{ft}$.

242. Saxifraga stellaris, Linn. [Lit. Brit. 23 ; Arct. 7, 36, 37 a, 38 ; Alps 2, 21 b.] At Clova the flower is variable, producing frequently extra parts; the terminal flower is especially so, large, and often has three carpels (cf. Wydler in Flora, I860, p. 387 ). This is partly in contrast to the observations of Warming and Schulz, who find the terminal flower is frequently female and small in size.

Visitors. Diptera. Empidae: (I) Empis lucida Ztt., 22. VI. 95, 2,600 ft. (2) E. vernalis Mg., ? sh. I2-I6. VI. 99, 22-2,500 ft. Anthomyiidae: (3) Drymia hamata Fln., sh. 20. VI. 96, 2,300 ft. (4) Anthomyia sulciventris Ztt., 22. VI. 95; 2 I V. 97, I0-I,800 ft. (5 \& 6) Anthomyia spp., sh. 1 9. VI.-6. VII. $95 ; 23$. V. 96 ; 20. VI.-6. VII. $96, \mathrm{I} 2-2,600 \mathrm{ft}$.

243. Chrysosplenium oppositifolium, Linn. [Lit. Brit. 23, 29; N.C.E. 1,3a.] Flowers $q$, by contabescence of the anthers, were observed once.

Visitors. Lepidoptera. Heterocera: (I) microlepidopteron, sh. 22. V. 96, I,200 ft. Hymenoptera. Petiolata parasitica: Ichneumonidae: (2) I sp., 22. V. 97 ; 1 4. V. 98, 5-900 ft. Diptera. Empidae: (3) Empis tessellata F., sh. 22. V. 96, I,200 ft. (4) Rhamphomyia sulcata Fln:, sh. 22. V. 96, r,200 ft. Cecidomyiidae: (5) Tanytarsus sp., sh. 14. V. 98; I 5. VI. 99, 9-1,900 ft. Mycetophilidae: (6) Sciara sp., 20. VI. 95, 2,500 ft. Psychodidae: (7) Psychoda sp., sh. I 4. V. 98, $900 \mathrm{ft}$. Anthomyiidae: (8) Anthomyia sulciventris Ztt., sh. 22. V. 97, $500 \mathrm{ft}$. (9) Anthomyia sp., 2 r. VI. 95, I, $400 \mathrm{ft}$. Cordyluridae: (10) Scatophaga stercoraria L., sh. I 4. V. 98, $900 \mathrm{ft}$. (I I) S. squalida Mg., 2 r. VI. 95, r,400 ft. Helomyzidae: (r 2) Helomyza flava Mg., sh. I2. V. $98,800 \mathrm{ft}$. Coleoptera. (13) Anthophagus alpinus Payk., 22. VI. $96,2,400 \mathrm{ft}$. (I 4) Telephorus paludosus Fln., sh. I 5. VI. 99, r,900 ft. Hemiptera. (i 5 ) I sp., sh. $22 . \mathrm{V} .97,500 \mathrm{ft}$.

\section{Class A, $\S 66$. Aucuparia Type.}

244. Pyrus aucuparia, Gaertn. [Lit. Brit. 23 ; N.C.E. 1, 3 b, 16, 18, 33, 40 ; de Vries 2460; Pyren. 17.)

Visitors. Lepidoptera. Heterocera: Tineidae: (I) I sp., I4. VI. 99, I,400 ft. Hymenoptera. Aculeata: Apidae: (2) Apis mellifica L., sh. I I. VI. 99, $800 \mathrm{ft}$. (3) Bombus terrestris L., ran rapidly over an inflorescence ? sh., I6. VI. 99, r,300 ft. (4) B. lapponicus F., I 4. VI. 99, I,400 ft. Formicidae: (6) Formica fusta Latr., I 4-r6. VI. 99, I, $400 \mathrm{ft}$. Petiolata tubulifera : Chrysididae: (6) Chrysis ? ignita L., I I. VI. 99, $800 \mathrm{ft}$. Petiolata parasitica : Chalcididae: (7) I sp., I 4. VI. 99, I,400 ft. 
Diptera. Syrphidae: (8) Syrphus vitripennis Mg., sh. I I-I6. VI. 99, 8-I,400 ft. (9) Chrysogaster hirtella Lw., I3. VI. 99, I,400 ft. (I0) Eristalis arbustorum L., I 3-I,400 ft. Empidae: (I I) Empis tessellata F., I6. VI. 99, I3-I,400 ft. (I 2 ) E. bilineata Lw., I I. VI. 99, $800 \mathrm{ft}$. (I 3) E. opaca F., I3. VI. 99, I,400 ft. (I 4) E. lucida Ztt., I3-I6. VI. 99', I3-I,400 ft. (I5) Rhamphomyia sulcata Fln., sh. I r. VI. 99, $800 \mathrm{ft}$. (16) R. cinerascens Mg., sh. I I. VI. 99, $800 \mathrm{ft}$. Chironomidae: (1 7 ) I sp., 28. VI. 95, I,800 ft. Bibionidae: (I 8) Scatopse sp., I4. VI. 99, I,400 ft. (I 9) Bibio nigriventris Hal., I3-I 4. VI. 99, I3-1,400 ft. Sarcophagidae: (20) Sarcophaga sp., I3. VI. 99, I,400 ft. Muscidae: (2 I) Lucilia sp., sh. I3-I4. VI. 99, I 3-I,400 ft. (2 2) Calliphora erythrocephala Mg., I I-I 6. VI. 99, I 3-I,400 ft. (23) C. vomitoria L., 1 4. VI. 99, I,400 ft. Anthomyiidae: (24) Mydaea sp., I I-I6. VI. 99, 8-I,400 ft. (25) Limnophora sp., 28. VI. 95, 1,800 ft. (26) Drymia hamata Fln., sh. 16. VI. 99, I,400 ft. (27) Trichophthicus sp., sh. 25 . VI. 95, 2,000 ft. Chloropidae: (28) Chlorops sp., I 4. VI. 99, I,400 ft. Coleoptera. (29) Meligethes viridescens F., sh. 25-28. VI. 95 ; I I-I 4. VI. 99, 8-2,000 ft. (30) Epuraea aestiva L., sh. 25. VI. 95, 2,000 ft. (3 I ) Corymbites cupreus F., I 4. VI. 99, I,400 ft. (32) C. quercus, Gyll., sh. I 4. VI. 99,

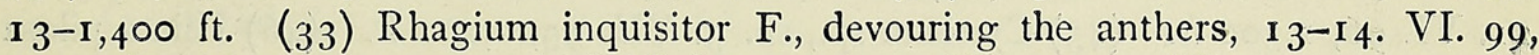
I,400 ft. Thysanoptera. (34) Thrips sp., sh. 25 . VI. 95 ; r 4 . VI. 99, I4-2,000 ft.

245. Crataegus Oxyacantha, Linn. [Lit. Brit. 23 ; N.C.E. 1, 3 b, 4, 14, 14 a, 16, 18, 34, 40 ; Pyren. 17.] Planted above $500 \mathrm{ft}$.

Visitors. Hymenoptera. Aculeata: Apidae: (r) Apis mellifica L., sh. Ir. VI. 99, $800 \mathrm{ft}$. Petiolata parasitica: Proctotrypidae: (2) I sp., I I. VI. 99, $800 \mathrm{ft}$. Diptera. Syrphidae: (3) Eristalis arbustorum L., sh. I9. VI. 99, $700 \mathrm{ft}$. Empidae: (4) Empis bilineata Lw., I I. VI. 99, $800 \mathrm{ft}$. (5) Rhamphomyia sulcata Fln., sh. I I-I 2. VI. 99, $800 \mathrm{ft}$. Bibionidae: (6) Scatopse brevicornis Mg., sh. I I-I 2. VI. 99, $800 \mathrm{ft}$. Coleoptera. (7) Meligethes viridescens F., sh. I I-I 9. VI. 99, 7-800 ft.

\section{Class A, $\S 67$. Alchemilla Type.}

246. Alchemilla alpina, Linn. [Lit. N.C.E. 1, 4, 34; Alps 2 ; Pyren. 17.] Very abundant.

Visitors. Lepidoptera. Rhopalocera: (I) Erebia epiphron Kn., 25. V. 95, once. Heterocera : Noctuidae: (2) Celaena haworthii Cuc., sh. 25. V.95; 2 I. IX. 95, 18-2,000 ft. Geometridae: (3) Larentia salicata Hb., I5-21. VI. 95, I 3-I,500 ft. Crambidae: (4) Pyrausta alpinalis Schiff, sh. I6-2 I. VI. 95, I 7-2,300 ft. Tortricidae: (5) Tortrix sp., sh. 19-2 7. VI. 95, I 7-2,400 ft. (6) Glyphipteryx fuscoviridella Haw., sh. I6. IX. 95, $900 \mathrm{ft}$. Tineidae: (7) Plutella cruciferarum Zel., sh. I9. VI. 95, 2,400 ft. Hymenoptera. Aculeata: Formicidae: (8) Formica fusca Latr., I6. VI.-r. VII. $95 ; 24$. IX. 95; 19-26. VI. 96; I 4. VI. 99, 9-2,100 ft. Myrmicidae: (9) Myrmica rubra L., sh. I6-2I. IX. 95, 9-I,300 ft. Sessiliventres: Tenthredinidae: (10) Tenthredo olivacea Klug., 25. VI. 95, 2,000 ft. (I I) Tenthredopsis sp., sh. I 7. VI. 99, I, 100 ft. (I 2) Dolerus elongatus Thomson, sh. I5-25. VI. 95; 22. VI. 96, I 2-2,400 ft. Petiolata parasitica: Ichneumonidae: (13) Ichneumon molitorius Grav., sh. I8-24. IX. 95, 9-2,400 ft. freq. (I 4) Alomyia debellator F., sh. 28. VI.-2. 
VII. $95 ; 2 \mathrm{I}-23$. IX. 95, 8-I,800 ft. (I 5) Pimpla examinator F., sh. 24. IX. 95, I 2-I,400 ft. (I 6) Limneria sp., sh. 2 I. IX. 95. I,300 ft. (I 7) Limneria sp., sh. 2 I-23. IX. 95, 2,200 ft. (I8) Polyrrhembia tenebricosa Grav., sh. 2 I. IX. 95, I,600 ft. (19) Hemiteles politus Bridgman, 2 I. IX. 95, r,700 ft. (20) H. micator Grav., I 9. IX. 95, 2,300 ft. (2 I) Hemiteles sp., I 5-20. VI. 95; I8-2 I. IX. 95, 8-r,600 ft. (22) Exephanes hilaris Grav., I9-23. IX. 95, I5-2,500 ft. (23) Campoplex sp., sh. 2 I. VI.-3. VII. 95, I I-2,200 ft. (24) Lissonotus sp., 25 . VI. 95, 2,200 ft. (25) Xylonomus?, sh. 20-23. IX. 95, 25-3,600 ft. (26) Pezomachus sp., 23. IX. 95, I,700 ft. (27 and 28 ) Two other spp., I 9. VI.-3. VII. 95; 20. IX. 95, 9-2,500 ft. Brachonidae: (29 and 30) Two spp., sh. 20. VI. 95 ; I6-24. IX. 95, 8-I,300 ft. Proctotrypidae: (3 I) Proctotrypes sp., 24. IX. 95, I,200 ft. Chalcididae: $\left(3^{2,}, 33,34\right.$, $35,3^{6}$, and 37 ) Six spp., sh. I 6-20. VI. 95 ; I 4-23. IX. 95 ; 1 8-3०. VI. 96, 8-2,500 ft. Cynipidae: (38) Eucoela sp., I4-23. IX. 95, I7-I,800 ft. Diptera. Syrphidae: (39) Syrphus sp., Io. VII. 96, 2,300 ft. (40) Ascia podagrica F., 23. IX. 95, 2,300 ft. Empidae: (4I) Empis tessellata F., sh. 2I-25. VI. 95, I5-2,000 ft. (42) E. aestiva Lw., sh. 30. VI. 96, 2, $100 \mathrm{ft}$. (43) E. lucida Ztt., 2 2. VI. 96, 2,400 ft. (44) Empis sp., 2 I. VI. 96, I,300 ft. (45) Cyrtoma sp., sh. 3. VII. 95, 2,200 ft. (46) Hilara pilosa Ztt., sh. 24. VI. 95, 2,000 ft. (47) Tachydromia pubicornis Ztt., 16-23. IX. 95, 9-I,600 ft. (48) T. stigmatella Ztt., 3. VII. 95, 2,400 ft., and 4. VII. 94, 2,000 ft. Cecidomyiidae: (49) Cecidomyia sp., 4. VII. 94. (50) Lestremia sp., sh. 2 I-23. IX. 95, I 2-2,000 ft. Mycetophilidae: (5 I) Sciara sp., sh. I6-24. VI. 95; I 4-23. IX. 95 ; I. VII. 96, 9-3,000 ft. freq. (52) Sceptonia nigra Mg., 4. VII. 94, ? 2,000 ft. (53) Boletina ? dubia Staeg., 23. IX. 95, 2, 100 ft. Bibionidae: (54) Dilophus albipennis Mg., sh. 2 I-2 5. VI. 95 ; 24. IX. 95; 27 . VI. 96, I I-2,200 ft. (55) Bibio pomonae F., sh. 23 . IX. 95 , 12-1,800 ft. Simuliidae: (56) Simulium sp., 25 . VI. 95 ; 16. IX. 95 , 9-1,800 ft. Chironomidae: (57) Corynoneura sp., I 9-20. IX. 95, 23-3,600 ft. (58) Chironomus sp., 20. VI. $95 ; 23$. IX. 95, I 8-I,900 ft. (59) Crichotopus sp., sh. 3. VII. 95, 2,200 ft. (60) Tanytarsus sp., 23. IX. 95, $700 \mathrm{ft}$. (6I) Metriocnemis sp., 23. IX. 95, I,400 ft. (62) Ceratopogon sp., 23. IX. 95, r,700 ft. Psychodidae: (63) Psychoda sp., I6-23. IX. 95, 9-1,800 ft. Limnobidae: (64) Rhypholophus nodulosus Mcq., sh. 23. IX. 95, 1,700 ft. Tipulidae: (65) Tipula excisa Schum., sh. I6-25. VI. 95, I9-2,400 ft. Dolichopodidae: (66) Dolichopus rupestris Hal., 6. VII. 95, 2,600 ft. (67) D. atratus Mg., 4. VII. 95, ? 2,000 ft. (68) Gymnopternus celer Mg., I6. IX. 95, $900 \mathrm{ft}$. Sarcophagidae: (69) Cynomyia mortuorum L., 21. VI. 95; 24. IX. 95, I 2-I,300 ft. Muscidae: (70) Jucilia cornicina F., sh. I9-24. IX. 95, 9-2,400 ft. (71) Calliphora erythrocephala Mg., 23-24. IX. 95, 9-1,800 ft. (72) Pollenia rudis F., I 9-24. IX. 95, 8-2,500 ft. freq. Anthomyizdae: (73) Hyetodesia incana W., sh. 20-28. VI. 95 ; 23. IX. 95 ; 19. VI. 96 ; 14-I 9. VI. 99, 8-2,500 ft. (74) H. semicinerea W., sh. I6. IX. 95, $900 \mathrm{ft}$. (75) H. basalis Ztt., 2 I. VI. 95, I, $500 \mathrm{ft} . \quad$ (76) H. sp., 4. VII. 95, 2,000 ft. (77) Mydaea ? tincta Ztt., 25. VI. 95, I,700 ft. (78) Spilogaster nigrivenis Ztt., sh. 27. VI. 96, I,400 ft. (79) S. quadrum F., sh. 24. VI. 95, I,500 ft. (80) Limnophora solitaria Ztt., 26. VI.-4. VI. 95; 19-27. VI. 96, 19-2,300 ft. (8I) Drymia hamata Fln., I6-25. VI. 95; 16. VI. 96, 13-2,200 ft. (82) Trichophthicus cunctans Mg., 4. VII. 95, ? 8,000 ft. (83 and 84) Trichophthicus spp., sh. 24. VI. 95 ; I 9. IX. 95, 20-2,200 ft. (85) Hylemyia nigrescens Rnd., sh. 24. VI. 95, I,700 ft. 
(86) Anthomyia sulciventris Ztt., 27. VI. 95, 2, 100 ft. (87, 88, and 89) Anthomyia spp., sh. I6. VI.-4. VII. 95 ; 2 I-2 4. IX. 95 ; 18. VI.-8. VII. 96, 8-2,700 ft. (90) Azelia aterrima Mg., I 7. VI. 95, 2,200 ft., and ? 4. VII. 95. (9I) Caricea sp., I 9. VI. 95, 2,400 ft. (92) Coenosia infantula Rnd., I. VII. 95, I, $200 \mathrm{ft.}$ (93) Coenosia sp., sh. 24. VI. 95, I,600 ft. Cordyluridae : (94) Scatophaga stercoraria L., ? fp. I 9. VI.-6. VII. 95 ; I6-24. IX. 95, 10-2,700 ft. (95) S. squalida Mg., 20-2 I. VI. 95, I3-I,900 ft. Sciomyzidae: (96) Tetanocera sp., I6. IX. 95, $900 \mathrm{ft}$. Ortalidae: (97) Pteropaectria frondescentiae L., I. VII. 96, I,900 ft. Sepsidae: (98) Sepsis cynipsea L., 23. IX. 95, I 6-I,900 ft. Ephydridae: (99) Hydrellia griseola Fln., 3. VII. 95, 2,600 ft. Chloropidae: (I00) Chlorops sp., 3. VII. 95, 2,600 ft. Borboridae: (I0I) Borborus equinus Fln., 23. IX. 95, I,800 ft. Phoridae: (1 02 ) Phora rufipes Mg., I 4. IX. 95, I, $200 \mathrm{ft}$. (I03) Phora sp., sh. 16. VI.-3. VII. 95; I6-24. IX. 95, 8-2,400 ft. freq. Coleoptera. (I04) Meligethes viridescens F., sh. 24 . VI. 95, I,600 ft. (I05) Anthophagus alpinus Payk., sh. 3-4. VII. 95; 22. VI.-6. VII. 96, 24-2,700 ft. (106) Epuraea aestiva L., 3. VII. 95, 2,500 ft. (107) Corymbetes cupreus F., 26. VI. 95, I,000 ft. (108) C. quercus Gyll., I 6-28. VI. 95, I 7-2,000 ft. (г 09 ) Dolopius marginatus L., 4. VII. 94. (I I 0 ) Helodes marginata F., I 5. VI. 95, I, 200 ft. (I I I) Sericosomus brunneus L., 25. VI. 95, I,700 ft. (I I 2) Telephorus paludosus Fln., I6.-20. VI. 95. (I I3) Rhagonycha limbata Thoms., sh. I7-24. VI. 95 ; I 9 . VI. 96 , I2-2,000 ft. (I I4) Malthodes atomus Thoms., I 8. VI. 96, I, I00 ft. (I I 5) Apion sp., I6. IX. 95, $900 \mathrm{ft}$. Hemiptera. (I I6) Heterocordylus tibialis Hahn., 25. VI. 95, $800 \mathrm{ft}$. (I I 7) Nabis flavomarginatus Scholtz, 2 I-23. IX. 95, 16-I,800 ft. (I I 8) Stygnus sp., 2 I. IX. 95, I,700 ft. ( I I 9) Psylla ?, sh. 2 I. IX. 95, I,800 ft. Neuroptera. (I 20 ) I sp., 20. VI. 95, I,700 ft. Collembola. (I 2 I) One springtail, sh. I 6-23. IX. 95, 9-I,500 ft. Araneida. (I 22 ) Xysticus?, lying in wait, 23 . IX. 95, I,700 ft. (I 23) Oligolophus morio F., sh. 2 I-2. IX. 95, 10-2,300 ft.

247. Alchemilla vulgaris, Lam. [Lit. Brit. $23 ;$ N.C.E. 1, 4, 9, 14, 21 b, Plateau 2002 ; Arct. 36 ; Alps 2, 16 ; Pyren. 17.] This begins to flower before $A$, alpina.

Visitors. Lepidoptera. Rhopalocera: ( $\mathrm{I}$ ) Coenonympha pamphilus L, I5. VI. 95, $800 \mathrm{ft}$. Heterocera: Geometridae: (2) Larentia salicata Hb., 20. VI. 95, 2,400 ft. Hymenoptera. Petiolata parasitica : Ichneumonidae: $(3,4$, and 5) 3 spp., sh. I 5. VI. 95 ; I6-2 2. IX. 95; 27. V. 97, 7-800 ft. Chalcididae: (6) I sp., I6-1 7 . IX. 95, $800 \mathrm{ft}$. Diptera. Empidae: (7) Empis lucida Ztt., I 5. VI. 95, $800 \mathrm{ft}$. Mycetophilidae: (8) Sciara sp., sh. 22. IX. 95, $800 \mathrm{ft}$. Chironomidae: (9) Ceratopogon leucopeza Mg., 22. IX. 95, $800 \mathrm{ft}$. (I0) A second sp., I3. V. 98, $800 \mathrm{ft}$. Tachinidae: (I I) Gymnochaete viridis Fln., sh. 22. V. 96, $800 \mathrm{ft}$. Muscidae: (1 2) Pollenia rudis F., sh. I 5-22. IX. 95, $800 \mathrm{ft}$. Anthomyiidae: (I3) Hyetodesia incana W., I 5-I 7. VI. 95 ; I 5. IX. 95, $800 \mathrm{ft}$. (I 4, I 5, and I6) Anthomyia spp., sh. I 7 . VI. 95 ; I 5-22. IX. 95 ; 19. V. 97 ; 15. V. $98,8-2,400$ ft. Cordyluridae: ( 7 ) Scatophaga stercoraria L., sh., 20. VI. $95 ;$ I $5^{-22}$. IX. $95 ; 22$. V. 96,800 , and once at $2,400 \mathrm{ft}$. Psilidae: (18) Psila?, I7. VI. 95, $800 \mathrm{ft}$. Trichaetidae: (19) Tephritis leontodontis, sh. 22. IX. 95, $800 \mathrm{ft}$. Phoridae: (20) Phora sp., I 5. IX. 95, $800 \mathrm{ft}$. Coleoptera. (21) Meligethes viridescens F., I6. IX. 95, 800 ft. (22) Enicmus minutus L.; 22. IX. 95, 
$800 \mathrm{ft}$. Neuroptera. (23) Chloroperla sp., 2 I. VI. 95, $800 \mathrm{ft}$. Hemiptera. (24) Aphis sp., I7. VI. 95, $800 \mathrm{ft}$.

\section{Class A, $\S 68$. Galium Type.}

248. Galium verum, Linn. [Lit. Brit. 23 ; N.C.E. 1, 3 c, 9, 14, 14 a, 18, 21 a, 31, 32, $40 ;$ Pyren. 17.]

Visitors. Lepidoptera. Heterocera: Eriocephalidae: (1) Eriocephala calthella L., sh. and fp. 4-20. VII. 95, $800 \mathrm{ft}$. Hymenoptera. Sessiliventres: Tenthredinidae: (2) Allantus arcuatus Forst., 5. VII. 95, $800 \mathrm{ft}$. (3) Abia sp., 6. VII. 95, $800 \mathrm{ft}$. Petiolata parasitica: Ichneumonidae: (4) I sp., 2. VII. 95, 800 ft. (5) 10. VII. 96, $900 \mathrm{ft}$. Diptera. Syrphidae: (6) Syritta pipiens L., sh. 23. VII. 95, $800 \mathrm{ft}$. Mycetophilidae: (7) Sciara sp., I6. IX. 95, $800 \mathrm{ft}$. Bibionidae: (8) Dilophus albipennis Mg., sh. 3. VII. 95, $800 \mathrm{ft}$. (9) Bibio pomonae F., I0-I I. VII. 96, 8-900 ft. Muscidae: (Io) Lucilia cornicina F., sh. 7. VII. 95, $800 \mathrm{ft}$. (I I) Calliphora erythrocephala Mg., sh. 5-23. VII. 95 ; I I. VII. 96, 800 ft. (I 2 ) Mesembryna meridiana L., sh. го. VII. 96, 800 ft. Anthomyizdae: (13) Hyetodesia incana W., sh. 3-23. VII. 95 ; I-II. VII. 96, 7-900 ft. (I4) Trichophthicus hirsutulus Ztt., sh. Io. VII. 96, $900 \mathrm{ft}$. (I5) Anthomyia sp., sh. ro. VII. 96, $900 \mathrm{ft}$. Sepsidae: (r6) Sepsis cynipsea L., 5. VII. 95, $800 \mathrm{ft}$. Chloropidae: (I 7 ) Oscinis sp., 5. VII. 95, $800 \mathrm{ft}$. Coleoptera. (18) Meligethes aeneus F., sh. 3. VII. 95, $800 \mathrm{ft}$. (I 9 ) Corymbites quercus Gyll., 2. VII. 95, $800 \mathrm{ft}$. (20) Serica brunnea L., 2. VII. 95, $800 \mathrm{ft}$. (2 I) Thyamis laevis Duft., 5. VII. 95, $800 \mathrm{ft}$.

249. Galium boreale, Linn. [Lit. N.C.E. 1,3 c, 21 a, 34; Alps 2.] Proterandrous, the stigmas not receptive until the anthers have dehisced and the stamens have bent back out of the way between the petals. This they do on the third day after the opening of the bud.

Visitors. Lepidoptera. Heterocera: Crambidae: (I) Pyrausta ? alpinalis Schiff., sh. 10. VII. 96, 2,000 ft. Hymenoptera. Aculeata: Formicidae: (2) Formica fusca Latr., sh. 6. VII. 95, I,800 ft. Petiolata parasitica : (3) I sp., 25 . VI. 96, $700 \mathrm{ft}$. Diptera. Syrphidae: (4) Platychirus manicatus Mg., sh. 25. VI. 96, $700 \mathrm{ft}$. (5) Syritta pipiens L., sh. I r. VII. 96, $700 \mathrm{ft}$. Bibionidae: (6) Bibio pomonae F., sh. I I. VII. 96, $700 \mathrm{ft}$. Orphnephilidae: (7) Orphnephila testacea Ruthé, Io. VII. 96, 2,000 ft. Anthomyizdae: (8) Hyetodesia variabilis Fln., sh. 25. VI. 96, $700 \mathrm{ft}$. (9) Spilogaster nigrivenis Ztt., sh. 25. VI. 96, 700 ft. (I0) Limnophora solitaria Ztt., sh. 26. VI.-IO. VII. 96, I9-2,I00 ft. (I I) Drymia hamata Fln., sh. I 5. VII. 95, 2,400 ft. (I 2 ) Anthomyia sulciventris Ztt., 25. VI. 96, $700 \mathrm{ft}$. (I3) A. sp., 6. VII. 96, I,700 ft. Cordyluridae: (I 4) Scatophaga sp., sh. 25 . VI. $96,700 \mathrm{ft}$.

250. Galium saxatille, Linn. (with G. sylvestre Poll.). [Lit. N.C.E. 1,3 c, 4, 14, 21 a, 21 b, 32, 33; Alps 2, 21 b.]

Visitors. Lepidoptera. Rhopalocera : (I) Argynnis aglaia L., sh. 25 . VI. 95, $800 \mathrm{ft}$. (2) Coenonympha pamphilus L., 25-28. VI. 95, 8-I 5, I00 ft. Heterocera : Geometres : (3) Psodos trepidaria Tr., sh. 29. VI. 95, 2,500 ft. Hymenoptera. 
Petiolata parasitica: Proctotrypidae: (4) Proctotrype sp., sh. 20. IX. 95, 3,700 ft. Diptera. Empidae: (5) Empis tessellata F., I. VII. 95, $800 \mathrm{ft}$. once. Psychodidae : (6) Psychoda?, sh. 22. IX. 95, 3,600 ft. Muscidae: (7) Lucilia cornicina F., 26. VI. 95, $800 \mathrm{ft}$. twice. (8) Calliphora vomitoria L., 3. VII. 95, $800 \mathrm{ft}$. once. (9) Pollenia rudis F., I6. VI. 95, $800 \mathrm{ft}$. once. Anthomyiidae: (10) Hyetodesia incana W., 26 . VI. 95, 800 ft. (I I) Limnophora solitaria Ztt., sh. 26. VI. 96, 2,200 ft. (I 2 ) Drymia hamata Fln., 25. VI.-3. VII. 95; 29. VI. 96, 8-2,600 ft. (I3) Trichophthicus sp., ? sh. 2. VII. 96, 2,800 ft. (I 4) Anthomyia sp., sh. I5-2 I. VI. 95 ; г о. VI.-8. VII. 96, 800 ft. Cordyluridae: (I 5) Scatophaga stercoraria L., sh. 2 I. VI. 95; 24. VI. 96, $800 \mathrm{ft}$. Ephydridae: (I6) Hydrellia griseola Fln., 3. VII. 95, I,600 ft. Chloropidae : ( 17 ) Chlorops ?, го. VII. 96, 2,200 ft. (I 8 ) Oscinis sp., 2 I. VII. 95, $800 \mathrm{ft}$. Coleoptera. (I 9) Corymbites cupreus F., sh. I 9. VI. 96, $900 \mathrm{ft}$.

251. Galium Aparine, Linn. [Lit. Brit. 23 ; N.C.E. 18, 33, 34.]

Visitors. Hymenoptera. Petiolata parasitica : Chalcididae: (I) I sp., sh. I-6. VII 95. Diptera. Anthomyiidae: (2) Coenosia sp., I. VII. 95. Both at $800 \mathrm{ft}$.

252. Galium palustre, Linn. [Lit. Brit. 23 ; N.C.E. 8, 14, 18, 25, 34.]

Visitors. Diptera. Empidae: (I) Empis tessellata F., sh. I. VII. 95. (2) E. stercorea L., sh. I. VII. 95. Muscidae: (3) Lucilia cornicina F., sh. r. VII. 95 . Anthomyiidae: (4) Hyetodesia incana W., 2-6. VII. 95. (5) H. variabilis Fln., I. VII. 95. All at $800 \mathrm{ft}$.

\section{Class A, $\S 69$. Arenaria sedoides Type.}

253. Arenaria sedcides, Schultz. [Lit. Alps 2, 21 b.] The flower opens very widely, so as to expose the abundant honey freely. The stamens bend very far out after dehiscing; but, as here and there an anther does not fall off, self-pollination may be brought about in the closing of the flower. Its duration is three to four days. The yellow nectaries are the most conspicuous part of the flower. Seed is freely produced.

Visitors. Hymenoptera. Petiolata parasitica: (I) I sp., sh. 27. VI. 96. Diptera. Mycetophilidae: (2) Sciara sp., sh. 19. VI. 99, and ? 2. VII. 96. Bibionidae: (3) Bibio nigriventris, Hal., sh. covered with pollen, 16. VI. 99. Anthomyiidae: (4) Anthomyia sp., I6. VI. 99. Cordyluridae: (5) Scatophaga stercoraria L., sh. I6. VI. 99. Sapromyzidae: (6) Sapromyza sp., I6. VI. 99. Coleoptera. (7) I sp. similar to Amara bifrons Gyll., sh. I6. VI. 99. Collembola. (8) I sp., sh. 2. VII. 96. Acarina. (9) I sp., sh. 2. VII. 96. All at 2,850 ft.

Class A attracted three species of the butterflies, three of the larger moths, and six of the smaller moths, including Eriocephala; of Hymenoptera, Apis, two species of Bombus, one of Andrena, one of Vespa, two species of ant, five of Tenthredinidae, one of Chrysis, and about thirty of parasitic 


\section{TABLE XLVIII.}

Actual number of Individuals visiting the flowers of Class A.

\begin{tabular}{|c|c|c|c|c|c|c|c|c|c|c|c|c|c|c|c|}
\hline & $\underset{\tilde{\Sigma}}{\vec{\Sigma}}$ & 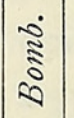 & 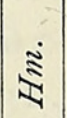 & 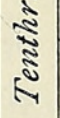 & 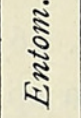 & $\stackrel{\square}{\S}$ & $\mid \begin{array}{c}\dot{0} \\
\tilde{c} \\
\tilde{\sigma} \\
\vdots\end{array}$ & $\begin{array}{c}\dot{a} \\
\dot{z} \\
v\end{array}$ & 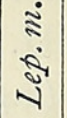 & $\stackrel{u}{2}$ & $\stackrel{2}{\Xi}$ & के & $\tilde{0}$ & 必 & है \\
\hline 240. Saxifraga aizoides. & - & 8 & I & 2 & 50 & I 30 & 6 & I & 3 & 46 & 29 & 309 & 25 & 3 & 6I3 \\
\hline 24I. Saxifraga umbrosa. . & - & - & - & - & I & - & - & - & - & - & $\mathrm{r}$ & 2 & - & - & 4 \\
\hline $\begin{array}{l}\text { 242. Saxifraga stellaris } \\
\text { 243. Chrysosplenium opposito- }\end{array}$ & - & - & - & - & - & - & - & - & - & - & 4 & 8 & - & - & 12 \\
\hline folium . . & - & - & - & - & 2 & - & - & - & I & - & I & I 8 & 2 & 2 & 26 \\
\hline 244. Pyrus Aucuparia & I & 2 & I & - & I & 3 & - & - & I & - & 33 & IIO & 79 & $5^{\mathrm{I}}$ & 282 \\
\hline 245. Crataegus Oxyacantha & I & - & - & - & I & - & - & - & - & - & 2 & 5 & 4 & - & I3 \\
\hline 246. Alchemilla alpina. . & - & - & - & 8 & 108 & 25 & - & 6 & 9 & - & 9 & 507 & $3^{I}$ & 25 & 728 \\
\hline 247. Alchemilla vulgaris & - & - & - & - & 6 & - & - & 2 & - & - & I & 82 & 3 & 2 & 96 \\
\hline 248. Galium verum . & - & - & - & 2 & 2 & - & - & - & - & 19 & I & 49 & 4 & - & 77 \\
\hline 249. Galium boreale. & - & - & - & - & I & I & - & - & 2 & - & 2 & 25 & - & - & $3 \mathbf{1}$ \\
\hline 250. Galium saxatile. & - & - & - & - & 3 & - & - & 4 & - & - & I & 29 & I & - & $3^{8}$ \\
\hline 25 I. Galium Aparine. & - & - & - & - & 3 & -- & - & - & - & - & - & 2 & - & - & 5 \\
\hline 252. Galium palustre. & - & - & -- & - & - & - & - & - & - & - & 4 & 6 & - & - & 10 \\
\hline 253. Arenaria sedoides . . & - & - & - & - & I & - & - & - & - & - & - & I 7 & I & 4 & 23 \\
\hline Total . & 2 & IO & 2 & I 2 & I 79 & I59 & 6 & I3 & I 6 & $6_{5}$ & 88 & I 69 & I 50 & 87 & $195^{8}$ \\
\hline Percentages & .10 & .51. & . Io & $.6 \mathrm{I}$ & $9 \cdot 15$ & $8 \cdot 13$ & 31 & .66 & 82 & $3 \cdot 3^{2}$ & $4 \cdot 5^{\circ}$ & $59 \cdot 73$ & $7 \cdot 67$ & $4 \cdot 45$ & \\
\hline
\end{tabular}

\section{TABLE XLIX.}

To show how great a proportion of injurious insects go to Class $\mathrm{A}$.

\begin{tabular}{|c|c|c|c|c|}
\hline & \multicolumn{2}{|c|}{ Available. } & \multicolumn{2}{|c|}{ To Class $A$. } \\
\hline & No. & $\%$ & No. & $\%$ \\
\hline Decidedly desirable & $I, 763$ & 10.19 & 25 & $\mathbf{I} \cdot 28$ \\
\hline Desirable. . & 1,277 & $7 \cdot 37$ & 106 & $5 \cdot 4 \mathrm{I}$ \\
\hline Indifferent & I 2,993 & 75.08 & $1,39^{\circ}$ & 70.98 \\
\hline Injurious . & 1,273 & $7 \cdot 3^{6}$ & 437 & $22 \cdot 3^{2}$ \\
\hline
\end{tabular}

TABLE L.

Insects visiting Class A in different seasons classed by desirability.

\begin{tabular}{|c|c|c|c|c|c|c|}
\hline & \multicolumn{2}{|c|}{ Spring. } & \multicolumn{2}{|c|}{ Summer. } & \multicolumn{2}{|c|}{ Autumn. } \\
\hline ly desirab & - & - & 22 & I. 66 & 3 & $\cdot 53$ \\
\hline Desira & 2 & 3.0 & 95 & $7 \cdot 18$ & 9 & 1.58 \\
\hline Indiffe & 59 & $89 \cdot 3$ & $93^{6}$ & $70 \cdot 75$ & 395 & $69 \cdot 4^{2}$ \\
\hline Injurious & 5 & $7 \cdot 57$ & 270 & $20.4 \mathrm{I}$ & 162 & $28 \cdot 47$ \\
\hline Total . & 66 & & 1,323 & & $5^{69}$ & \\
\hline
\end{tabular}


TABLE LI.

Percentages of different groups of insects visiting Class A in different seasons.

\begin{tabular}{|c|c|c|c|c|c|c|c|c|c|c|c|c|}
\hline & $\stackrel{\tilde{n}}{\tilde{2}}$ & § & $\lesssim$ & 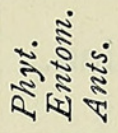 & 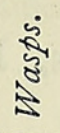 & ¿ँ & $\begin{array}{l}\text { ลे } \\
\text { ปे }\end{array}$ & $\dot{\vec{\nu}}$ & $\approx$ & $\tilde{a}$ & ¿ & 匈 \\
\hline Sprin & - & $\bar{c}$ & - & $4 \cdot 54$ & - & - & I. 5 I & - & $\mathrm{I} \cdot 5^{\mathrm{I}}$ & 89.39 & - & 3.03 \\
\hline Summer & $\cdot{ }^{\prime} 5_{5}$ & .60 & - I 5 & I 5.94 & $\cdot 45$ & .90 & $\cdot 5^{2}$ & $4 \cdot 9 \mathrm{I}$ & $6.5^{\circ}$ & $54 \cdot 7^{2}$ & $10.6_{5}$ & 4.45 \\
\hline Autumn & - & $\cdot 35$ & - & 23.90 & & - 17 & $I \cdot 40$ & - & - I 7 & 67.83 & I. $5^{8}$ & 4.56 \\
\hline
\end{tabular}

TABLE LII.

Desirability of the visitors to flowers of Class A, according to colour, in percentages.

\begin{tabular}{|c|c|c|c|}
\hline & Yellow. & White. & Green. \\
\hline Decidedly desirable & $\mathrm{I} \cdot 26$ & 2.03 & $\cdot 94$ \\
\hline Desirable. & 5.03 & I $2.9 \mathrm{I}$ & $2 \cdot 24$ \\
\hline Indifferent & $66 \cdot 76$ & $68 \cdot 6 \mathrm{r}$ & 75.68 \\
\hline Injurious & $26 \cdot 95$ & $16 \cdot 45$ & $2 \mathrm{I} \cdot \mathrm{I} 3$ \\
\hline
\end{tabular}

TABLE LIII.

Percentages of different groups of insects visiting flowers of different colours in Class A.

\begin{tabular}{|c|c|c|c|c|c|c|c|c|c|c|c|c|}
\hline & $\vec{\pi}$ & §ั & 䏒 & 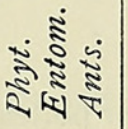 & 3 & ปัن & \begin{tabular}{l} 
今 \\
\multirow{3}{*}{}
\end{tabular} & $\dot{4}$ & 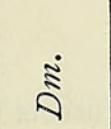 & $\ddot{\theta}$ & i & 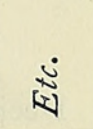 \\
\hline $\begin{array}{l}\text { Yellow } \\
\text { White. } \\
\text { Green. }\end{array}$ & $\overline{5}-\overline{5}$ & $\begin{array}{c}\text { I.II } \\
\underbrace{\circ}\end{array}$ & $\begin{array}{l}.13 \\
.25 \\
-\end{array}$ & $\begin{array}{r}26.25 \\
3.44 \\
17 \cdot 47\end{array}$ & $\begin{array}{l}.83 \\
- \\
-\end{array}$ & $\begin{array}{r}. \text { I3 } \\
\text { I.OI } \\
.94\end{array}$ & $\begin{array}{r}\cdot 55 \\
.75 \\
\mathrm{I} .06\end{array}$ & $\begin{array}{c}9.07 \\
=\end{array}$ & $\begin{array}{r}4.32 \\
11.89 \\
1.18\end{array}$ & $\begin{array}{l}52 \cdot 52 \\
47 \cdot 34 \\
71 \cdot 54\end{array}$ & $\begin{array}{r}4 \cdot 32 \\
21 \cdot 26 \\
4 \cdot 13\end{array}$ & $\begin{array}{r}.69 \\
\mathbf{1} 2.9 \mathrm{I} \\
3.65\end{array}$ \\
\hline
\end{tabular}

Hymenoptera ; among the Diptera, in Syrphidae, of one species of Eristalis, of Sericomyia, of Chilosia, and of Chrysogaster, of three species of Syrphus, and one of each of the following: Platychirus, Syritta, Sphaerophoria, and Ascia ; in Empidae, of eight species of Empis, and of the same number of short-tongued Empids; in Tachinidae, only of Echinomyia and Gymnochaeta viridis, and not of Siphona ; in Muscidae, of three species of Calliphora and one each of Lucilia, Mesembryna, and Pollenia ; in Sarcophagidae, of two species of Cynomyia and one Sarcophaga; in Anthomyiidae, of twenty-seven species, including Drymia hamata; in Tipulidae, of two species; in Bibionidae, of four; in Cordyluridae, of two species of Scatophaga ; and of thirty-two species of the smaller flies, including almost all the Dolichopids observed ; among Coleoptera, of twenty species ; among Hemiptera, of five species; among Neuroptera, of two ; among Thysanoptera, of one ; among Collembola, of one; in Acarina, of one ; and in Araneida, of two. 
A brief review of the insects visiting the massed flowers of Class A may be found earlier.

\section{Class Po. A, $\S 70$. A-like Type.}

254. Chenopodium Bonus-Henricus, Linn. [Lit. N.C.E. 1, 14, 18, 21 b, 34; Warming 2490.

Visitor. Coleoptera. (I) Oxytelus sp., ?fp. 19. VI. 99, $800 \mathrm{ft}$.

The only visitors to Class Po. $\S$ A were two individuals of a beetle.

\section{TABLE LIV.}

Total number of insect visitors to A-like flowers of Class Po., and total of visitors to B-like, $\mathrm{AB}-$ like, and A-like flowers, taken together.

\begin{tabular}{|c|c|c|c|c|c|c|c|c|c|c|c|c|c|c|c|}
\hline Class Po. $(A)$. & $\stackrel{\bar{\pi}}{\bar{\nabla}}$ & $\begin{array}{l}\stackrel{0}{\Xi} \\
\vdots\end{array}$ & 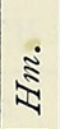 & $\frac{\text { वे }}{3}$ & 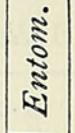 & 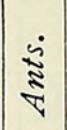 & $\begin{array}{c}\dot{s} \\
\vdots \\
\vdots \\
\vdots\end{array}$ & ¿ें & 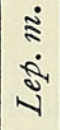 & में & 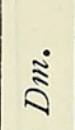 & $\dot{\theta}$ & i & 选 & हैं \\
\hline $\begin{array}{l}\text { 254. Chenopodium Bonus- } \\
\text { Henricus . . . }\end{array}$ & - & - & - & - & - & - & - & - & - & - & - & - & 2 & - & 2 \\
\hline $\begin{array}{l}\text { Total of all flowers } \\
\text { of B-like, AB-like, } \\
\text { and A-like Po. . }\end{array}$ & $5^{6}$ & I 4 & 2 & I & 1 & I & $\mathbf{I}$ & - & I & 18 & 23 & 344 & 5 & 6 & 473 \\
\hline Percentages & II .84 & $2 \cdot 96$ & $\cdot 4^{2}$ & .21 & $|.2 \mathrm{I}|$ & $.2 \mathrm{I}$ & .21 & - & $.2 \mathrm{I}$ & $3 \cdot 8 I$ & $4 \cdot 86$ & $72 \cdot 73$ & I. 06 & $I \cdot 27$ & \\
\hline
\end{tabular}

TABLE LV.

Decidedly desirable insects visit honeyless flowers (for pollen) in rather more than the class's share ; injurious insects are rare on them.

\begin{tabular}{|c|c|c|c|c|}
\hline & \multicolumn{2}{|c|}{ Available. } & \multicolumn{2}{|c|}{ To Po. (all forms). } \\
\hline & No. & $\%$ & No. & $\%$ \\
\hline Decic & I, $7^{6} 3$ & 10.19 & 70 & 14.80 \\
\hline & 1,277 & $7 \cdot 37$ & 26 & $5 \cdot 50$ \\
\hline Indifferent & I 2,993 & 75.08 & $3^{68}$ & 77.80 \\
\hline Injurious . & $\mathrm{I}, 273$ & $7 \cdot 36$ & 9 & I. 90 \\
\hline
\end{tabular}

TABLE LVI.

Insects visiting Class Po. (all three divisions taken together) in different seasons by desirability.

\begin{tabular}{|c|c|c|c|c|c|c|}
\hline & \multicolumn{2}{|c|}{ Spring. } & \multicolumn{2}{|c|}{ Summer. } & \multicolumn{2}{|c|}{ Autumn. } \\
\hline Decidedly desirable & I 2 & $5 \cdot 50$ & 58 & 23.67 & - & - \\
\hline Desirable & 3 & I. 37 & 23 & $9 \cdot 39$ & - & - \\
\hline Indifferent & 203 & 93. I I & 155 & $63 \cdot 27$ & IO & $100 \cdot 00$ \\
\hline Injurious . & - & - & 9 & 3.67 & - & - \\
\hline Tot & 218 & & 245 & & I0 & \\
\hline
\end{tabular}


TABLE LVII.

Percentages of different groups of insects visiting honeyless flowers (Class Po.) in the different seasons.

\begin{tabular}{|c|c|c|c|c|c|c|c|c|c|c|c|c|}
\hline & 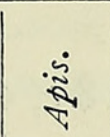 & §ั & 핓 & हैं & s & نَّ & 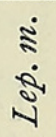 & 它 & 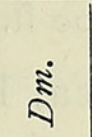 & $\ddot{\Delta}$ & $\overrightarrow{0}$ & 㒖 \\
\hline $\begin{array}{l}\text { Spring } \\
\text { Summer } \\
\text { Autumn }\end{array}$ & $\begin{array}{r}3 \cdot 67 \\
19.59 \\
-\end{array}$ & $\begin{array}{l}I .84 \\
4.08\end{array}$ & $\overline{.82}$ & $\frac{-}{1 \cdot 22}$ & $\overline{._{4} \mathrm{I}}$ & $\begin{array}{l}\bar{z} \\
\bar{z}\end{array}$ &.$\overline{4}$ & $\overline{7 \cdot 35}$ & $\begin{array}{l}\mathrm{I} \cdot 3^{8} \\
.16\end{array}$ & $\begin{array}{r}95 \cdot 12 \\
53.47 \\
100.00\end{array}$ & $\begin{array}{c}- \\
2.04 \\
-\end{array}$ & $\frac{-}{2 \cdot 45}$ \\
\hline
\end{tabular}

TABLE LVIII.

Desirability of visitors to honeyless flowers (Class Po.) according to colour, in percentages.

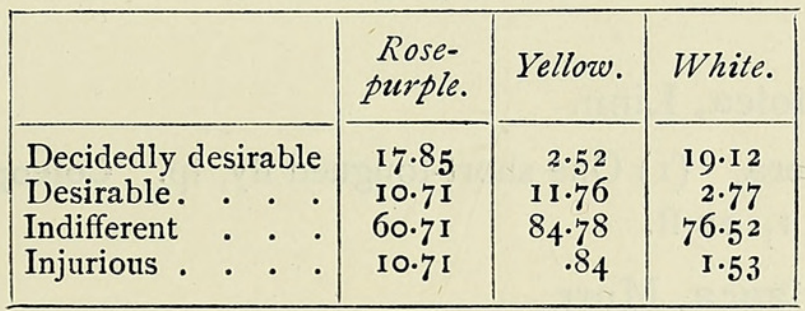

TABLE LIX.

Percentages of different groups of insects visiting honeyless flowers (Class Po.) of different colours.

\begin{tabular}{|c|c|c|c|c|c|c|c|c|c|c|c|c|}
\hline & ₹ & 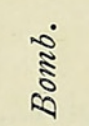 & 胥 & 这这 & 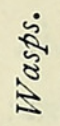 & 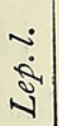 & న̃ & $\begin{array}{l}\dot{y} \\
\stackrel{\Delta}{ل}\end{array}$ & $\approx$ & $\ddot{a}$ & $\ddot{0}$ & खิ \\
\hline Rose-purple & - & $\begin{array}{r}7.85 \\
.84\end{array}$ & $-\overline{68}$ & $\overline{8}$ & - & - & $\overline{8}$ & - & I0.7 I & 60.71 & - & $10.7 \mathrm{I}$ \\
\hline $\begin{array}{l}\text { Yellow : } \\
\text { White : }\end{array}$ & $\begin{array}{r}16.60 \\
16.66\end{array}$ & $\begin{array}{r}.04 \\
2 \cdot 46\end{array}$ & 1.08 & $\begin{array}{l}.04 \\
.61\end{array}$ & .30 & - & .84 & $\begin{array}{r}10.08 \\
1.85\end{array}$ & $\begin{array}{l}9 \cdot 24 \\
2 \cdot 77\end{array}$ & $73 \cdot 45$ &.$\overline{92}$ & .92 \\
\hline
\end{tabular}

Class Po. was visited by a Tortrix and by Eriocephala calthella ; among Hymenoptera, by Apis, by three species of Bombus, by one of Andrena, one wasp, one ant, one Tenthredine, and one Chalcidid; among Diptera, in Syrphidae, by two species of Eristalis, by one each of Syrphus, Chrysogaster, Ascia, and Sphaerophoria, and by three of Platychirus ; in Empidae, only by Empis tessellata, and then only on a single occasion; in Muscidae, by a Calliphora and a Lucilia; by thirteen species of Anthomyiidae; by two species of Scatophaga, a Dilophus, and by four other small flies; by three Coleoptera ; one Neuroptera ; and in Thrysanoptera, by Thrips. 
Class W, $\S 7 \mathrm{I}$.

255. Plantago lanceolata, Linn. [Lit. Brit. 23, Darwin 485 ; N.C.E. $1,3 \mathrm{a}, 3 \mathrm{~b}, 3 \mathrm{c}, 4,8,9,10,11,12,14,18,21 \mathrm{a}, 21 \mathrm{~b}, 30,33,34$.

Visitor. Hymenoptera. Aculeata: Vespidae: (I) Vespa sylvestris Scop., seeking h. 4. VII. 95 , $900 \mathrm{ft}$.

256. Urtica dioica, Linn. [Lit. N.C.E. 1, 3 a, 3 b, 3 c, 4, 8, 9, 10, 11, $12,14,18,34$.) Vegetative reproduction seems to continue uninterruptedly.

Visitors. Diptera. Syrphidae: (I) Chrysogaster hirtella Lw., sitting on flowers, I5. VII. 95, $800 \mathrm{ft}$. Cordyluridae: (2) Scatophaga stercoraria L., on flowers, 24 . VI. 95, 800 ft. Anthomyiidae: (3) Azelia sp., fp. 21. VI. 95, $800 \mathrm{ft}$. Coleoptera. (4) Brachypterus urticae F., fp. 2 I.-24. VI. 95, 8-900 ft. freq.

257. Betula alba, Linn. [Lit. N.C.E. 14, 18.]

Visitor. Diptera. Syrphidae: ( I) Syrphus punctulatus Verrall, fp. Io. V. 97 $800 \mathrm{ft}$.

258. Carex dioica, Linn.

Visitors. Diptera. (I) One short-tongued fly, fp. Coleoptera. (2) One sp., fp. Both 22. V. 96, 1, 100 ft.

259. Carex glauca, Murr.

Visitor. Diptera. Anthomyiidae: (I) Anthomyia sulciventris Ztt., fp. 22. V.97, $600 \mathrm{ft}$.

26o. Alopecurus pratensis, Linn. [Lit. N.C.E. 14, 18, 34.] $800 \mathrm{ft}$.

Visitor. Diptera. Syrphidae: (I) Platychirus manicatus Mg., fp. I 5. VI. 99,

26I. Abies excelsa, Poiret. [Lit. N.C.E. 34.]

Visitor. Lepidoptera. Rhopalocera: (I) Pieris napi L., once hovering about young female cones, apparently seeking h., I I. VI. 99, $900 \mathrm{ft}$.

TABLE LX.

Total number of individuals visiting wind-fertilized flowers, Class W.

\begin{tabular}{|c|c|c|c|c|c|c|c|c|c|c|c|c|c|c|c|}
\hline & \begin{tabular}{l}
$\dot{n}$ \\
\multirow{2}{*}{}
\end{tabular} & §ँ & $\stackrel{\Sigma}{\Sigma}$ & 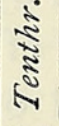 & 咅 & $\frac{\dot{\Omega}}{\Sigma}$ & $5^{2}$ & ¿ّं & 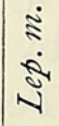 & نे & $\approx$ & $\ddot{\imath}$ & $\tilde{0}$ & 㝴 & $\begin{array}{l}\tilde{\Xi} \\
\text { ¿ี }\end{array}$ \\
\hline 255. Plantago lanceolata & - & - & - & - & - & - & I & - & $1-$ & - & - & - & - & - & I \\
\hline${ }_{25}^{25}$. Urtica dioica. . & - & - & - & - & - & - & - & - & - & - & I & 5 & 53 & - & 59 \\
\hline 257. Betula alba. & - & - & - & - & - & - & - & - & - & - & I & - & - & - & I \\
\hline 258. Carex dioica... & - & - & - & - & - & - & - & - & - & - & - & I & 2 & - & 3 \\
\hline 259. Carex glauca . . & - & - & - & - & - & - & - & - & - & - & - & I & - & - & I \\
\hline 260. Alopecurus pratensis & - & - & - & - & - & - & - & - & - & - & I & - & - & - & I \\
\hline 26I. Abies excelsa. . . & - & - & - & - & - & - & - & I & - & - & - & - & - & - & I \\
\hline Total . . & - & - & - & - & - & - & I & I & - & - & 3 & 7 & 55 & - & 67 \\
\hline Percentage . & - & - & - & - & - & - & I. 49 & I. 49 & - & - & $4 \cdot 4^{8}$ & 10.45 & 82.09 & - & - \\
\hline
\end{tabular}


The flowers of Class $\mathrm{W}$ deceived into visiting them one butterfly and one wasp; they also received the visits, among Diptera, of three Syrphids, a Scatophaga, an Anthomyia, and an Azelia ; and among Coleoptera, of two species.

We abstain in this paper from comparing our results with those of Muiller, MacLeod, and others; the comparison will follow in the last part of our paper, wherein too we shall discuss fully the results of our work. 


\section{$2 \mathrm{BHL}$ Biodiversity Heritage Library}

Willis, J. C. and Burkill. I. H. 1908. "Flowers and insects in Great Britain part IV." Annals of botany 22, 603-649.

https://doi.org/10.1093/oxfordjournals.aob.a089192.

View This Item Online: https://www.biodiversitylibrary.org/item/232525

DOI: https://doi.org/10.1093/oxfordjournals.aob.a089192

Permalink: https://www.biodiversitylibrary.org/partpdf/318928

\section{Holding Institution}

Smithsonian Libraries

\section{Sponsored by}

Biodiversity Heritage Library

\section{Copyright \& Reuse}

Copyright Status: Not in copyright. The BHL knows of no copyright restrictions on this item.

This document was created from content at the Biodiversity Heritage Library, the world's largest open access digital library for biodiversity literature and archives. Visit BHL at https://www.biodiversitylibrary.org. 